Pontifícia Universidade $C_{\text {atólica }}$

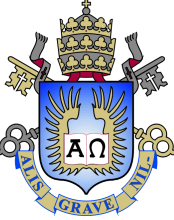

Isabela Vasconcellos Viana

\title{
An Analytical Model for Injectivity Tests in Multilayered Reservoirs with Formation \\ Crossflow
}

Dissertação de Mestrado

Thesis presented to the Programa de Pós-graduação em Matemática, do Departamento de Matemática da PUC-Rio in partial fulfillment of the requirements for the degree of Mestre em Matemática.

Advisor :

Prof. Sinesio Pesco

Co-advisor: Prof. Abelardo Borges Barreto Junior 


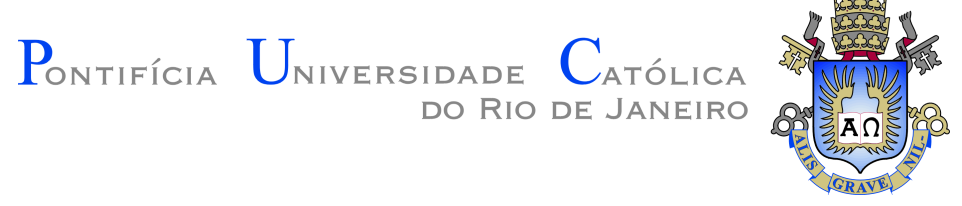

Isabela Vasconcellos Viana

\section{An Analytical Model for Injectivity Tests in Multilayered Reservoirs with Formation \\ Crossflow}

Thesis presented to the Programa de Pós-graduação em Matemática da PUC-Rio in partial fulfillment of the requirements for the degree of Mestre em Matemática. Approved by the Examination Committee:

Prof. Sinesio Pesco

Advisor

Departamento de Matemática - PUC-Rio

Prof. Abelardo Borges Barreto Junior Co-advisor

Departamento de Matemática - PUC-Rio

Prof. Márcio da Silveira Carvalho

Departamento de Engenharia Mecânica - DEM/PUC-Rio

Dra. Priscila Magalhães Ribeiro

Centro de Pesquisa e Desenvolvimento Leopoldo Américo

Miguez de Mello - CENPES/Petrobras

Rio de Janeiro, April the 16th, 2021 
All rights reserved.

\section{Isabela Vasconcellos Viana}

Graduated in Mathematics from Federal Fluminense University (UFF) in 2018.

Bibliographic data

Viana, I.V.

An Analytical Model for Injectivity Tests in Multilayered Reservoirs with Formation Crossflow / Isabela Vasconcellos Viana; advisor: Sinesio Pesco; co-advisor: Abelardo Borges Barreto Junior. - 2021.

92 f: il. color. ; $30 \mathrm{~cm}$

Dissertação (mestrado) - Pontifícia Universidade Católica do Rio de Janeiro, Departamento de Matemática, 2021.

Inclui bibliografia

1. Matemática - Teses. 2. Matemática - Teses. 3. Teste de Injetividade. 4. Reservatório Multicamadas. 5. Fluxo Cruzado de Formação. 6. Reservatório Composto. 7. Modelo Analítico. I. Pesco, S.. II. Borges Barreto Junior, A.. III. Pontifícia Universidade Católica do Rio de Janeiro. Departamento de Matemática. IV. Título. 


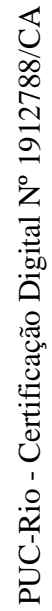

To my parents Elaine and Paulo. 


\section{Acknowledgments}

I would like to thank very much my advisor Professor Sinesio Pesco and my co-advisor Professor Abelardo Barreto Jr. for all their guideness, patience and support.

To my parents Elaine and Paulo, my sister Luiza, my fiancé Jônatas, my grandmothers Cida and Peia and all my family for their love and faith in me.

To my amazing friends and colleagues in PUC-Rio, in special: Camila, Giovanna, Raphael, Guilherme, Jessica and Renan for both the study and the fun moments.

To Professor Cecilia Fernandez and Professor Ana Maria Luz at UFF for the opportunity of being a part of the project Mulheres na Matemática.

To Conselho Nacional de Desenvolvimento Científico e Tecnológico $(\mathrm{CNPq})$ and Petrobras for their financial support.

This study was financed in part by the Coordenação de Aperfeiçoamento de Pessoal de Nível Superior - Brasil(CAPES) - Finance Code 001. Therefore, I would like to thank CAPES.

Finally, I would like to thank PUC-Rio for the scholarship grant and Departamento de Matemática in PUC-Rio for the environment that encourages pleasant study. 


\section{Abstract}

Viana, I.V.; Pesco, S. (Advisor); Borges Barreto Junior, A. (CoAdvisor). An Analytical Model for Injectivity Tests in Multilayered Reservoirs with Formation Crossflow. Rio de Janeiro, 2021. 92p. Dissertação de Mestrado - Departamento de Matemática, Pontifícia Universidade Católica do Rio de Janeiro.

The injectivity test consists of injecting a phase, usually water, into an oil reservoir in order to collect information about it. Knowing these reservoir's parameters can be valuable in order to improve oil production. Many studies have been presented regarding the behavior of pressure in multilayered reservoirs under single phase fluid flow and, also, during injectivity tests. However, an analytical solution for pressure behavior in multilayered reservoirs during injectivity tests is well known only when the formation crossflow is not considered. Therefore, the present work attempts to develop an analytical model in the Laplace space for multilayered radially composite reservoirs with formation crossflow under single phase fluid flow, and then, for multilayered reservoirs with formation crossflow under two phase fluid flow. The accuracy of the proposed solution was verified by comparison with a finite difference flow simulator. The results provided by the analytical model and by the numerical data were consistently similar. Furthermore, the data obtained by the analytical solution was used to estimate the reservoir's equivalent permeability. Calculated values presented a satisfactory accuracy for all cases.

\section{Keywords}

Injectivity Test; Multilayered Reservoir; Formation Crossflow; Composite Reservoir; Analytical Model. 


\section{Resumo}

Viana, I.V.; Pesco, S.; Borges Barreto Junior, A.. Um Modelo

Analítico para Testes de Injetividade em Reservatórios Multicamadas com Fluxo Cruzado de Formação. Rio de Janeiro, 2021. 92p. Dissertação de Mestrado - Departamento de Matemática, Pontifícia Universidade Católica do Rio de Janeiro.

O teste de injetividade consiste em injetar uma fase, usualmente água, em um reservatório de óleo para coletar informações sobre ele. Conhecer os parâmetros do reservatório pode ser valioso para melhorar a produção de óleo. Muitos estudos têm sido apresentados a respeito do comportamento da pressão em reservatórios multicamadas sob escoamento de fluxo monofásico e, também, durante os testes de injetividade. No entanto, uma solução analítica para o comportamento da pressão em reservatórios de múltiplas camadas durante os testes de injetividade é bem conhecida apenas quando o fluxo cruzado de formação não é considerado. Portanto, o presente trabalho apresenta um modelo analítico no espaço de Laplace para reservatórios radialmente compostos multicamadas considerando o fluxo cruzado de formação sob fluxo monofásico e, então, para reservatórios multicamadas com fluxo cruzado de formação sob fluxo bifásico. A precisão da solução proposta foi verificada através da comparação com um simulador numérico de fluxo. Os resultados fornecidos pelo modelo analítico e pelos dados numéricos foram consistentemente semelhantes. Além disso, os dados obtidos pela solução analítica foram utilizados para estimar a permeabilidade equivalente do reservatório. Os valores calculados apresentaram uma aproximação satisfatória para todos os casos.

\section{Palavras-chave}

Teste de Injetividade; Reservatório Multicamadas; Fluxo Cruzado de Formação; Reservatório Composto; Modelo Analítico. 


\section{Table of contents}

$\begin{array}{lll}1 & \text { Introduction } & 13\end{array}$

2 Previous Achievements $\quad 15$

2.1 Reservoir Systems Under Single Phase Flow $\quad 15$

2.2 Reservoir Systems Under Two Phase Flow 16

3 Pressure Response Considering Formation Crossflow in Multilayered Reservoirs Under Single Phase Flow with Two Regions per Layer

3.1 Two Layered Model with Two Regions of Equal Radii per Layer 18

3.2 Multilayered Model with Two Regions of Equal Radii per Layer 29

3.3 Two Layered Model with Two Regions of Distinct Radii per Layer 32

3.4 Formation Damage 40

4 Pressure Response Considering Formation Crossflow in Multilayered Reservoirs Under Single Phase Flow with Multiple Regions per Layer $\quad 43$

4.1 Model Description $\quad 43$

4.2 Mathematical Formulation 46

5 Pressure Response Considering Formation Crossflow in Multilayered Reservoirs Under Two Phase Flow 51

5.1 Two Layered Model $\quad 51$

$\begin{array}{lll}5.2 & \text { Multilayered Model } & 60\end{array}$

6 Results and Discussion $\quad 62$

6.1 Cases Under Single Phase Flow 63

6.2 Cases Under Two Phase Flow 69

$\begin{array}{lll}6.3 & \text { Equivalent Permeability } & 76\end{array}$

7 Conclusions and Suggestions for Future Work 80

$\begin{array}{lr}\text { Bibliography } & 81\end{array}$

A Pressure solution in the Laplace's field 83

A.1 Some Properties on the Laplace's Transform 83

A.2 Some Properties on the Bessel's Functions 84

A.3 Stehfest's Algorithm 86

B Multilayered Case Under Single Phase Flow with Formation Crossflow and a Single Region of Permeability per Layer 88

C Semipermeability Coefficients Number $\quad 92$ 


\section{List of figures}

Figure 3.1 Reservoir with two layers and two regions of equal radii 19

Figure 3.2 Reservoir with two layers and two regions of different radii 33

Figure 3.3 3 layers with 4 regions of equal radii created artificially $\quad 40$

Figure 3.4 Damage region of radius $r_{s}$ and permeability $k_{s} \quad 40$

Figure 3.5 Representation of damage factor $\Delta p_{\text {skin }} \quad 41$

Figure 3.6 Two layered reservoir with damaged region near the wellbore 42

Figure 4.1 Reservoir with $n$ connected layers and many regions 44

Figure $4.2 \alpha$ extensions of the regions in each layer 45

Figure 5.1 Two phase fluid flow in a reservoir 52

Figure 5.2 Water injection behavior at layer 1 (left) and layer 2 (right) 53

Figure 6.1 Analytical and numerical pressure variation and derivative solutions for case $A_{1}$

Figure 6.2 Analytical and numerical pressure variation and derivative solutions for case $B_{1}$

Figure 6.3 Analytical and numerical pressure variation and derivative solutions for case $C_{1}$

Figure 6.4 Analytical and numerical pressure variation and derivative solutions for case $D_{1}$

Figure 6.5 Analytical and numerical pressure variation and derivative solutions for case $E_{1}$

Figure 6.6 Analytical and numerical pressure variation and derivative solutions for case $F_{1}$

Figure 6.7 Analytical and numerical pressure variation and derivative solutions for case $A_{2}$

Figure 6.8 Analytical and numerical pressure variation and derivative solutions for case $B_{2}$

Figure 6.9 Analytical and numerical pressure variation and derivative solutions for case $C_{2}$

Figure 6.10 Analytical and numerical pressure variation and derivative solutions for case $D_{2}$

Figure 6.11 Analytical and numerical pressure variation and derivative solutions for case $E_{2}$

Figure 6.12 Analytical and numerical pressure variation and derivative solutions for case $F_{2}$

Figure A.1 Modified Bessel's functions

Figure C.1 Case where $\alpha=4$ 


\section{List of tables}

Table 6.1 Analyzed single-phase flow cases 63

Table 6.2 Analyzed two phase fluid flow cases 70

Table 6.3 Real and estimated equivalent permeability values and $\begin{array}{ll}\text { percentage error for all cases } & 77\end{array}$

Table 6.4 Semilog graphs for the single phase flow cases 78

$\begin{array}{lll}\text { Table 6.5 Semilog graphs for the two phase flow cases } & 79\end{array}$ 


\section{List of Abbreviations}

$A_{j}^{i}, B_{j}^{i}$ - Coefficients for $j t h$ layer and $i t h$ region defined in the pressure solution $B_{w}$ - Water formation volume factor

$c_{t}$ - Total system compressibility

$f_{f j}$ - Fractional flow of phase $F$ in layer $j$

$h_{j}$ - Thickness of layer $j$

$h_{T}$ - Total reservoir thickness

$I_{0,1}, K_{0,1}$ - Modified Bessel functions of the first and second kind

$i_{j}$ - Region $i$ in layer $j$

$k_{j}$ - Horizontal permeability in layer $j$

$k_{e q}$ - Reservoir equivalent permeability

$k_{j}^{i}$ - Horizontal permeability in layer $j$ and region $i$

$k_{v_{j}}^{i}$ - Permeability of shale between layers $j$ and $j+1$

$k_{z_{j}}^{i}$ - Vertical permeability in layer $j$ and region $i$

$m_{j}$ - Number of regions in layer $j$

$M\left(j_{1}: j_{n}, i_{1}: i_{m}\right)$ - Matrix's lines $j_{1}$ through $j_{n}$ and columns $i_{1}$ through $i_{m}$ $n$ - Number of layers in the reservoir system

$o$ - Oil

$p_{i}$ - Initial Pressure

$p_{j i}$ - Reservoir pressure in layer $j$ and region $i$

$\bar{p}$ - Pressure change in Laplace domain

$p_{w f}$ - Well-bottom hole pressure

$p_{\text {skin }}$ - Pressure of damaged well-bottom hole

$q$ - Surface production flow rate

$q_{j i}$ - Flow rate for layer $j$ and region $i$

$r$ - Radius 
$r_{j}^{i}$ - Radius of region $i$ in layer $j$

$r_{j F}-$ Water front radius in layer $j$

$r_{e}$ - Reservoir outer radius

$r_{w}$ - Wellbore radius

$S$ - Skin factor

$S_{w i}^{j}$ - Irreducible water saturation in layer $j$

$S_{o r}^{j}$ - Residual oil saturation in layer $j$

$S_{F}^{j}$ - Saturation of fluid $F$ in layer $j$

$t$ - Time

$u$ - Laplace variable

$w$ - Water

$X_{j}^{i}$ - Semipermeability between layers $j$ and $j+1$ in region $i$

$\Delta h_{j}$ - Thickness of shale between layers $j$ and $j+1$

$\Delta p$ - Pressure change

$\Delta p_{\text {skin }}$ - Pressure change with skin effect along the well

$\chi_{j}^{i}$ - Permeability-thickness ratio

$\kappa_{j}^{i}$ - Permeability-thickness product for layer $j$ and region $i$

$\hat{\lambda}_{j}^{F}$ - Endpoint mobility of phase $F$ in layer $j$

$\phi_{j}$ - Porosity of layer $j$

$\mu_{j}$ - Viscosity of layer $j$

$\mu_{j}^{F}$ - Viscosity related to phase $\mathrm{F}$ in layer $j$

$\omega_{j}$ - Porosity-thickness product for layer $j$ 


\section{Introduction}

An injectivity test consists on injecting water into an oil reservoir and in response to that, many significant information about the reservoir can be provided, which can be valuable in order to improve the oil production. For instance, equivalent permeability, outer boundary condition and recoverable oil volume can be inferred. The way of detecting those information is through analyzing the pressure transient response. In this work, an analytical solution, which provides the pressure change solution, will be presented for two relevant cases of the reservoir's disposal, they are: single phase fluid flow in multilayered radially composite reservoirs and two phase fluid flow in multilayered reservoirs. For both of these cases, formation crossflow is considered.

Formation crossflow, different than wellbore crossflow, happens when there is a nonzero vertical permeability at the interface between two adjacent layers. This condition will be considered in all of the work proposed through the chapters. Besides formation crossflow, this work will consider a multilayered reservoir system, where all layers properties, such as porosity, permeability and thickness, may be different in each layer. In addition, different regions of permeability, in the same layer, are considered all along the model for the single phase fluid flow case, that is a radially composite reservoir system.

In chapter 2 , an overview on the work that was previously done regarding single phase flow in multilayered systems with formation crossflow and two phase flow in multilayered systems without formation crossflow, is presented. The present work combines these two topics.

The first case considered in this work, extended in chapters 3 and 4, considers a multilayered composite reservoir model under single phase fluid flow, that is, a production well is considered. It also considers formation crossflow along all layers. In chapter 3, 2 regions of different permeability are considered in each layer and in chapter 4 , many regions are considered. A solution for a composite reservoir system can be used to consider formation damage in the reservoir, also it can be extended to obtain a two phase model further on. In the last session of chapter 3, formation damage is considered.

The second and main case is described in chapter 5 . A multilayered model with formation crossflow is considered as well, however, for this case the flow 
considered will be a two phase flow. This problem is solved similarly to the single phase flow in composite reservoirs presented in chapter 3. For this case, skin effect will be disregarded at all times. A single permeability region is considered for each layer. However, layer properties may variate from each other.

For both models, an analysis of the particular case considering two layers will be made, in order to facilitate the understanding, before moving onto the multilayered case model, which obviously works for a two layered model as well. The suggested mathematical formulation in this work is mostly done in the Laplace Domain, where the pressure variation solution is well known in terms of Bessel's functions. Then, the solution in the real field is computed by the Stehfest Algorithm. All of the deduction presented in this work was made in consistent units.

The results comparing the model suggested in this work and the numerical simulator are shown in chapter 6. Lastly, chapter 7 consists of the conclusions and suggestions for future works. 


\section{2}

\section{Previous Achievements}

This chapter will present some of the previous works on single phase fluid flow in single and multilayered systems, with and without formation crossflow, and on two phase fluid flow in multilayered systems without formation crossflow. The present work combines these topics, hence, this chapter will be helpful in order to familiarize with them.

\section{1}

\section{Reservoir Systems Under Single Phase Flow}

One important work in single-phase flow in stratified reservoir system, which inspired many others was the one by Lefkovits et al.[10]. In this work, an analytical model was proposed, where the properties, such as thickness, permeability, and porosity could be different in each layer. Formation damage was also considered. However, formation crossflow is not considered in that work, only wellbore crossflow, which is when there is communication between two layers strictly through the well. Such reservoirs are denoted as commingled systems. On the other hand, the works by Economides and Joseph[9], R. Prijambodo et al.[13] and Gao and Deans[15] included formation crossflow.

The work proposed by Economides and Joseph[9], was strongly based on the previous [10], and an analytic model is presented in it, also considering a multilayered reservoir under single phase flow, however this work included formation crossflow. Properties may also be different from layer to layer. The presented model considers both formation damage and wellbore storage, and a derivation was made for both short and long times. This work showed that after a period of time, the reservoir could be described as an equivalent single layer system. This very complete work was the main study source for this present work. The formulation regarding the interflow between layers was derived from it.

In addition, the work presented by Economides and Joseph[9] used numerical inversion of Laplace transforms, more specifically, the Stehfest Algorithm[14] in order to find a pressure response in the real field. The first work to use it, was the one proposed by Tariq and Ramey[16]. Besides that contribution, Tariq and Ramey's[16] work also included an introduction to 
wellbore storage.

The study presented by Prijambodo et al.[13], considered a two layer cylindrical reservoir. It also considered damaged regions for the results. It presents an analysis for producer wells. It showed that the flowing pressure response at the well at early times can be divided in three flow periods. The first one shows that the reservoir behaves as if there were no crossflow, the second one, a transitional period, shows that the pressure response depends on the contrast in horizontal permeabilities and on the degree of communication between layers. During the third period, the reservoir is equivalent to a singlelayer system, like the results presented in [9] and [15].

Gao and Deans[15] proposed a complete work on multilayered reservoirs with formation crossflow which contained different cases of reservoir's systems. Chapters 2 through 6 of the work proposed by Gao and Deans[15], included pressure responses and interpretation theory for drawdown and buildup tests for two-layer and multilayered reservoirs under single phase fluid flow.

The work proposed by Bourdet et al.[5], was all done in the real field. This work proposes an analytic model considering formation crossflow, for a two layer reservoir. It also considers wellbore storage and skin effects. In addition, it includes a double porosity and permeability model description.

A solution regarding composite radial reservoir's model was proposed by Closmann et al. [8]. This work considered a single layered model and it did not consider formation crossflow.

\section{2}

\section{Reservoir Systems Under Two Phase Flow}

The two phase flow region in each layer considered in this present work, was based on the work done by Barreto Jr. et al.[3]. Which had the same goal, however following a different approach from the work by Bratvold and Horne[6].

Barreto Jr. et al.[3] considered a multilayered reservoir and presented a pressure solution for the injection period. Darcy's law was used to obtain an expression to calculate the pressure change. Their solution for the injection period assumes, like in this present work, a radially infinite reservoir.

Bratvold and Horne[6] work consisted in studying the influence of temperature on fluid mobility and on saturation gradients. Their formulation was also based on the discretization of the saturation profile proposed by BuckleyLeverett[7]. The work of Bratvold and Horne[6] included solutions for both injection and falloff periods. It also included storage effect and formation damage. 
The approach used in this present work, to reach the same goal as in [3] and [6], was proposed by Neto et al. [12] which extended the solution from a model for a radially composed reservoir under single phase flow as in given by Neto et al. [11], because during injectivity tests in a homogeneous reservoir, there are still different regions, but in respect to the fluids. The formulation proposed by [12] was done in the Laplace domain and considered a singlelayered model.

The work presented by Gahtani et al.[2], also included a formulation developed in the Laplace field. It considered a composite reservoir model, with an index to assess the impact of the mobility. The importance of considering the impact of water flow on formation has also been reported.

Finally, Bonafé et al.[4] presented a new formulation to approximate the pressure response at the well during injectivity tests. The solution considered multiple flow rates in a single layer reservoir. The proposed formulation is also used to determine the reservoir permeability at any specific injection or falloff period.

None of the previous works discussed in this section considered formation crossflow, unlike this present work, which proposes a formulation for multilayered systems, under two phase flow, with formation crossflow. 


\section{Pressure Response Considering Formation Crossflow in Multi- layered Reservoirs Under Single Phase Flow with Two Regions per Layer}

In this chapter, pressure response for a reservoir containing 2 different regions of permeability in all layers is introduced. There are two main cases to consider; The first one supposes that the radii of the regions in the layers are equal and the second on that they can be distinct.

The mathematical formulation for each one of these cases, considering formation crossflow, is presented. Throughout the equations, the variable $j$ will index the layer and the variable $i$, the region.

The case considering formation crossflow in a multilayered reservoir with a single region of permeability is presented in Appendix B.

\section{1}

\section{Two Layered Model with Two Regions of Equal Radii per Layer}

In this section, a reservoir with two layers and two regions of permeability is considered and the radii $\left(r_{j i}\right)$ of the regions in layer 1 are equal to the ones in layer 2.

Each layer is considered to be homogeneous for all properties but permeability, laterally isotropic, has a single phase flow of viscosity $(\mu)$ that is constant, and also has constant small total compressibility $\left(c_{t}\right)$. Layer properties, such as permeability $\left(k_{j i}\right)$, thickness $\left(h_{j}\right)$ and porosity $\left(\phi_{j}\right)$, may be different.

In addition, vertical crossflow may occur in both regions, so there are two semipermeability coefficients $\left(X_{1}^{i}\right)$ between the layers in each region $i$, which will be defined further on. Figure 3.1 illustrates this model: 


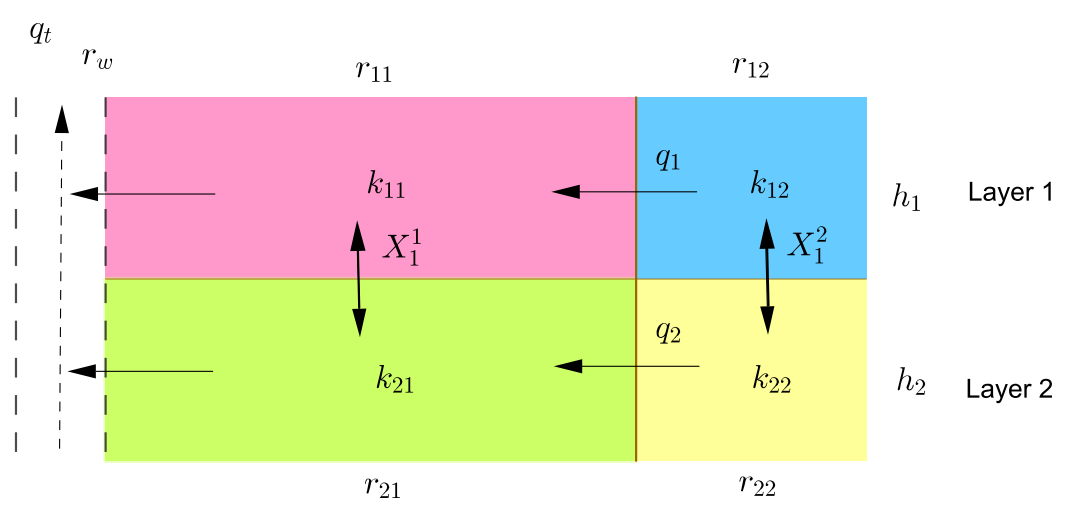

Figure 3.1: Reservoir with two layers and two regions of equal radii

Considering all of these hypothesis, the Diffusion Equation and its suitable boundary conditions will model this problem. Which are:

- The Initial Condition (I.C); which occurs at time $t=0$ and reflects that the reservoir is in equilibrium, that is, the pressure is the same in all layers.

- The Internal Boundary Condition (I.B.C) which is related to the way in which the well is producing during the test.

- The External Boundary Condition (E.B.C) which refers to the flow behavior at the extreme limit of the reservoir. In this case, it will be considered a laterally infinite reservoir.

The following system of equations define the reservoir model. Considering $\kappa_{j i}=k_{j i} h_{j}, \omega_{j}=\phi_{j} h_{j}$ and $\Delta p_{j i}=p_{i}-p_{j i}$ :

\section{Layer 1:}

Region 1:

$$
\begin{aligned}
& P D E: \quad \kappa_{11} \nabla^{2} \Delta p_{11}=\omega_{1} c_{t} \mu \frac{\partial \Delta p_{11}}{\partial t}-\left(\Delta p_{21}-\Delta p_{11}\right) X_{1}^{1} \\
& I C: \quad \Delta p_{11}(r, 0)=0 \\
& I B C: \quad q_{11}=\left.\frac{2 \pi \kappa_{11} r}{\mu}\left(\frac{\partial \Delta p_{11}}{\partial r}\right)\right|_{r=r_{w}}
\end{aligned}
$$


Region 2:

$$
\begin{aligned}
& \text { PDE }: \quad \kappa_{12} \nabla^{2} \Delta p_{12}=\omega_{1} c_{t} \mu \frac{\partial \Delta p_{12}}{\partial t}-\left(\Delta p_{22}-\Delta p_{12}\right) X_{1}^{2} \\
& I C: \Delta p_{12}(r, 0)=0 \\
& E B C: \quad \lim _{r \rightarrow \infty} \Delta p_{12}(r, t)=0
\end{aligned}
$$

The coefficient of semipermeability, $X_{j}^{i}$, between layers $j$ and $j+1$, in region $i$, is defined as presented in [9], but now considering a coefficient for each region of permeability in each layer $j$ :

$$
X_{j}^{i}=\frac{2}{2\left[\left(\Delta h_{j}\right) / k_{v j}^{i}\right]+\chi_{j+1}^{i}+\chi_{j}^{i}}
$$

Here $X_{2}^{i}=0 . \Delta h_{j}$ and $k_{v_{j}}^{i}$ are the thickness and the permeability of shale between layers $j$ and $j+1$ in region $i$ and $\chi_{j}^{i}$ is defined as:

$$
\chi_{j}^{i}=\frac{h_{j}}{k_{z_{j}}^{i}}
$$

Where $k_{z_{j}}^{i}$ is the vertical permeability of region $i$ in layer $j$. If there is no formation crossflow between layers 1 and 2 then $X_{1}$ is zero.

There are coupling conditions relating the regions, which must be defined in order to solve the problem properly. The coupling conditions between the regions $(\mathrm{CCR})$ are given by the pressure and flow rate equality at the interface between them:

$$
\left\{\begin{array}{l}
\Delta p_{11}\left(r=r_{11}, t\right)=\Delta p_{12}\left(r=r_{11}, t\right) \\
q_{11}=q_{12}
\end{array}\right.
$$

Using Darcy's law it is possible to rewrite the flow rate relation of the CCR so that all equations depend only on the pressure variation: 


$$
\left\{\begin{array}{l}
\Delta p_{11}\left(r=r_{11}, t\right)=\Delta p_{12}\left(r=r_{11}, t\right) \\
\left.\left(r \frac{\partial \Delta p_{11}}{\partial r}\right)\right|_{r=r_{11}}=\left.\frac{\kappa_{12}}{\kappa_{11}}\left(r \frac{\partial \Delta p_{12}}{\partial r}\right)\right|_{r=r_{11}}
\end{array}\right.
$$

\section{Layer 2:}

Region 1:

$$
\begin{aligned}
& \text { PDE : } \quad \kappa_{21} \nabla^{2} \Delta p_{21}=\omega_{2} c_{t} \mu \frac{\partial \Delta p_{21}}{\partial t}+\left(\Delta p_{21}-\Delta p_{11}\right) X_{1}^{1} \\
& I C: \quad \Delta p_{21}(r, 0)=0 \\
& I B C: \quad q_{21}=\left.\frac{2 \pi \kappa_{21} r}{\mu}\left(\frac{\partial \Delta p_{21}}{\partial r}\right)\right|_{r=r_{w}}
\end{aligned}
$$

Region 2:

$$
\begin{aligned}
& P D E: \quad \kappa_{22} \nabla^{2} \Delta p_{22}=\omega_{2} c_{t} \mu \frac{\partial \Delta p_{22}}{\partial t}+\left(\Delta p_{22}-\Delta p_{12}\right) X_{1}^{2} \\
& I C: \quad \Delta p_{22}(r, 0)=0 \\
& E B C: \quad \lim _{r \rightarrow \infty} \Delta p_{22}(r, t)=0
\end{aligned}
$$

As in layer 1, the layer 2 coupling conditions between regions is defined:

$$
\left\{\begin{array}{c}
\Delta p_{21}\left(r=r_{21}, t\right)=\Delta p_{22}\left(r=r_{21}, t\right) \\
\left.\left(r \frac{\partial \Delta p_{21}}{\partial r}\right)\right|_{r=r_{21}}=\left.\frac{\kappa_{22}}{\kappa_{21}}\left(r \frac{\partial \Delta p_{22}}{\partial r}\right)\right|_{r=r_{21}}
\end{array}\right.
$$

Between layers, there are also coupling conditions, the CCL. These conditions are obtained considering that the pressure is equal in the interface between the layers along the well and that the flow rate at the well is given by the sum of the flow rates of each layer, that is: 


$$
\left\{\begin{array}{l}
\Delta p_{11}\left(r=r_{w}, t\right)=\Delta p_{21}\left(r=r_{w}, t\right) \\
q=q_{1}+q_{2}
\end{array}\right.
$$

Using Darcy's law, the CCL is given as:

$$
\left\{\begin{array}{c}
\Delta p_{11}\left(r=r_{w}, t\right)=\Delta p_{21}\left(r=r_{w}, t\right) \\
\frac{q \mu}{2 \pi r_{w}}=\left.\frac{\partial \Delta p_{11}}{\partial r}\right|_{r=r_{w}}+\left.\frac{\partial \Delta p_{21}}{\partial r}\right|_{r=r_{w}}
\end{array}\right.
$$

To solve the problem presented above, the solution is first given in the Laplace field using Bessel's functions. The properties used both on Laplace transforms and on Bessel's functions are described in Appendix A.1 and A.2. The Stehfest Algorithm[14], which is described in Appendix A.3, is then used to find the solution in the real field.

Consider $\Delta \bar{p}$ to be the Laplace's transform of the pressure variation and $u$ to be the Laplace's variable. Applying the Laplace transform in equations (3.1.1) to (3.1.19):

\section{Layer 1:}

Region 1:

$$
\begin{aligned}
& \text { PDE : } \quad \kappa_{11} \nabla^{2} \Delta \bar{p}_{11}=\omega_{1} c_{t} \mu u \Delta \bar{p}_{11}-X_{1}^{1}\left(\Delta \bar{p}_{21}-\Delta \bar{p}_{11}\right) \\
& I C: \Delta \bar{p}_{11}(r, t=0)=0 \\
& I B C: \quad \bar{q}_{11}=\left.\frac{2 \pi \kappa_{11} r}{\mu}\left(\frac{\partial \Delta \bar{p}_{11}}{\partial r}\right)\right|_{r=r_{w}}
\end{aligned}
$$

Region 2:

$$
\begin{aligned}
& P D E: \quad \kappa_{12} \nabla^{2} \Delta \bar{p}_{12}=\omega_{1} c_{t} \mu u \Delta \bar{p}_{12}-X_{1}^{2}\left(\Delta \bar{p}_{22}-\Delta \bar{p}_{12}\right) \\
& I C: \Delta \bar{p}_{12}(r, t=0)=0 \\
& E B C: \quad \lim _{r \rightarrow \infty} \Delta \bar{p}_{12}(r, t)=0
\end{aligned}
$$


And the CCR,

$$
\left\{\begin{array}{l}
\Delta \bar{p}_{11}\left(r=r_{11}, t\right)=\Delta \bar{p}_{12}\left(r=r_{11}, t\right) \\
\left.\left(r \frac{\partial \Delta \bar{p}_{11}}{\partial r}\right)\right|_{r=r_{11}}=\left.\frac{k_{12}}{k_{11}}\left(r \frac{\partial \Delta \bar{p}_{12}}{\partial r}\right)\right|_{r=r_{11}}
\end{array}\right.
$$

\section{Layer 2:}

Region 1:

$$
\begin{aligned}
& P D E: \quad \kappa_{21} \nabla^{2} \Delta \bar{p}_{21}=\omega_{2} c_{t} \mu u \Delta \bar{p}_{21}+X_{1}^{1}\left(\Delta \bar{p}_{21}-\Delta \bar{p}_{11}\right) \\
& I C: \quad \Delta \bar{p}_{21}(r, t=0)=0 \\
& I B C: \quad \bar{q}_{21}=\left.\frac{2 \pi \kappa_{21} r}{\mu}\left(\frac{\partial \Delta \bar{p}_{21}}{\partial r}\right)\right|_{r=r_{w}}
\end{aligned}
$$

Region 2:

$$
\begin{aligned}
& \text { PDE : } \kappa_{22} \nabla^{2} \Delta \bar{p}_{22}=\omega_{2} c_{t} \mu u \Delta \bar{p}_{22}+X_{1}^{2}\left(\Delta \bar{p}_{22}-\Delta \bar{p}_{12}\right) \\
& I C: \Delta \bar{p}_{22}(r, t=0)=0 \\
& E B C: \quad \lim _{r \rightarrow \infty} \Delta \bar{p}_{22}(r, t)=0
\end{aligned}
$$

And the CCR,

$$
\left\{\begin{array}{l}
\Delta \bar{p}_{21}\left(r=r_{21}, t\right)=\Delta \bar{p}_{22}\left(r=r_{21}, t\right) \\
\left.\left(r \frac{\partial \Delta \bar{p}_{21}}{\partial r}\right)\right|_{r=r_{21}}=\left.\frac{k_{22}}{k_{21}}\left(r \frac{\partial \Delta \bar{p}_{22}}{\partial r}\right)\right|_{r=r_{21}}
\end{array}\right.
$$


The CCL is given by:

$$
\left\{\begin{array}{l}
\Delta \bar{p}_{11}\left(r=r_{w}, t\right)=\Delta \bar{p}_{21}\left(r=r_{w}, t\right) \\
\frac{q \mu}{2 \pi u}=\left.\left(\kappa_{11} r \frac{\partial \Delta \bar{p}_{11}}{\partial r}\right)\right|_{r=r_{w}}+\left.\left(\kappa_{21} r \frac{\partial \Delta \bar{p}_{21}}{\partial r}\right)\right|_{r=r_{w}}
\end{array}\right.
$$

The general pressure solution in the Laplace field for this problem is given by:

Region 1:

$$
\Delta \bar{p}_{j 1}=A_{j}^{1} K_{0}\left(\sigma_{j 1} r\right)+B_{j}^{1} I_{0}\left(\sigma_{j 1} r\right)
$$

Region 2:

$$
\Delta \bar{p}_{j 2}=A_{j}^{2} K_{0}\left(\sigma_{j 2} r\right)+B_{j}^{2} I_{0}\left(\sigma_{j 2} r\right)
$$

For $j=1,2 . A_{j}^{i}$ and $B_{j}^{i}$ are coefficients to be determined. Applying the laplacian in (3.1.35) and (3.1.36) the following is obtained:

$$
\nabla^{2} \Delta \bar{p}_{j i}=\sigma^{2} \Delta \bar{p}_{j i}
$$

This equivalence (3.1.37) is replaced in the PDE (3.1.20) for region 1 in layer 1:

$$
\kappa_{11} \sigma^{2} \Delta \bar{p}_{11}=\omega_{1} c_{t} \mu u \Delta \bar{p}_{11}-X_{1}^{1}\left(\Delta \bar{p}_{21}-\Delta \bar{p}_{11}\right)
$$

And rearranging equation (3.1.38) the following equation is obtained:

$$
\left(\kappa_{11} \sigma_{11}^{2}-\omega_{1} c_{t} \mu u-X_{1}^{1}\right) \Delta \bar{p}_{11}+X_{1}^{1} \Delta \bar{p}_{21}=0
$$


Likewise, replacing (3.1.37) in the PDE (3.1.23) for region 2 in layer 1:

$$
\left(\kappa_{12} \sigma_{12}^{2}-\omega_{1} c_{t} \mu u-X_{1}^{2}\right) \Delta \bar{p}_{12}+X_{1}^{2} \Delta \bar{p}_{22}=0
$$

And finally, replacing (3.1.37) in the PDE's (3.1.27) and (3.1.30) for regions 1 and 2 in layer 2 :

$$
\left(\kappa_{21} \sigma_{21}^{2}-\omega_{2} c_{t} \mu u-X_{1}^{1}\right) \Delta \bar{p}_{21}+X_{1}^{1} \Delta \bar{p}_{11}=0
$$

$$
\left(\kappa_{22} \sigma_{22}^{2}-\omega_{2} c_{t} \mu u-X_{1}^{2}\right) \Delta \bar{p}_{22}+X_{1}^{2} \Delta \bar{p}_{12}=0
$$

Equations (3.1.39) and (3.1.41) and equations (3.1.40) and (3.1.42) form two homogeneous system (one for each region $i$ ), where the non-trivial solution is wanted (i.e $\Delta \bar{p}_{j i} \neq 0$ ):

$$
\left[\begin{array}{cc}
\kappa_{1 i} \sigma_{1 i}^{2}-\omega_{1} c_{t} \mu u-X_{1}^{i} & X_{1}^{i} \\
X_{1}^{i} & \kappa_{2 i} \sigma_{2 i}^{2}-\omega_{2} c_{t} \mu u-X_{1}^{i}
\end{array}\right]\left[\begin{array}{c}
\Delta \bar{p}_{1 i} \\
\Delta \bar{p}_{2 i}
\end{array}\right]=0
$$

Each one of these two systems has a non-trivial solution, if and only if, the matrix of the coefficients in (3.1.43), say $T$, is singular, and, consequently, its determinant must be zero. The values of $\sigma$ must be found in order to satisfy this.

Equalizing the determinant of $T$ to zero in order to find the values of the $\sigma$ roots is equivalent to calculate the eigenvalues $\kappa_{j i} \sigma^{2}$ from matrix $K$ :

$$
K=\left[\begin{array}{cc}
\omega_{1} c_{t} \mu u+X_{1}^{i} & -X_{1}^{i} \\
-X_{1}^{i} & \omega_{2} c_{t} \mu u+X_{1}^{i}
\end{array}\right]
$$


That is because, the following is true:

$$
T=\operatorname{eig}(K) I-K
$$

Consequently, $\operatorname{det}(T)=0$ implies that $\operatorname{det}(\operatorname{eig}(K) I-K)=0$. Where $I$ is the identity matrix. Then, to find $\sigma$, it is left to extract the square root of the eigenvalues of $K$ divided by $\kappa_{j i}$ :

$$
\sigma_{j i}=\sqrt{\frac{e i g(K)}{\kappa_{j i}}}
$$

Now, with the values of the $\sigma$ determined by (3.1.46), it is left to find the values of the pressure coefficients.

Applying the EBC in the general solution for region 2 (3.1.36):

$$
\lim _{r \rightarrow \infty}\left[A_{j}^{2} K_{0}\left(\sigma_{j 2} r\right)+B_{j}^{2} I_{0}\left(\sigma_{j 2} r\right)\right]=0
$$

And knowing the properties described in Appendix A.2 for Bessel's modified functions, it is true that:

$$
\lim _{r \rightarrow \infty}\left[B_{j}^{2} I_{0}\left(\sigma_{j 2} r\right)\right]=0 \Longleftrightarrow B_{j}^{2}=0
$$

Hence, the pressure solution for region 2 is given by:

$$
\Delta \bar{p}_{j 2}(r, u)=A_{j}^{2} K_{0}\left(\sigma_{j 2} r\right)
$$

Using the pressure solutions (3.1.49) and (3.1.35) in the pressure CCR: 


$$
A_{j}^{1} K_{0}\left(r_{j 1} \sigma_{j 1}\right)+B_{j}^{1} I_{0}\left(r_{j 1} \sigma_{j 1}\right)=A_{j}^{2} K_{0}\left(r_{j 1} \sigma_{j 2}\right)
$$

Another relation for both layers can be obtained from the flow rate CCR:

$$
\kappa_{j 1} \sigma_{j 1}\left(A_{j}^{1} K_{1}\left(r_{j 1} \sigma_{j 1}\right)-B_{j}^{1} I_{1}\left(r_{j 1} \sigma_{j 1}\right)\right)=\kappa_{j 2} \sigma_{j 2}\left(A_{j}^{2} K_{1}\left(r_{j 1} \sigma_{j 2}\right)\right)
$$

$$
A_{j}^{1} K_{1}\left(r_{j 1} \sigma_{j 1}\right)-B_{j}^{1} I_{1}\left(r_{j 1} \sigma_{j 1}\right)=\frac{\kappa_{j 2}}{\kappa_{j 1}} \frac{\sigma_{j 2}}{\sigma_{j 1}}\left[A_{j}^{2} K_{1}\left(r_{j 1} \sigma_{j 2}\right)\right]
$$

By the pressure relation in the CCL, it can be stated that:

$$
A_{1}^{1} K_{0}\left(r_{w} \sigma_{11}\right)+B_{1}^{1} I_{0}\left(r_{w} \sigma_{11}\right)=A_{2}^{1} K_{0}\left(r_{w} \sigma_{21}\right)+B_{2}^{1} I_{0}\left(r_{w} \sigma_{21}\right)
$$

Replacing (3.1.35) in the IBC for layers 1 and 2:

$$
\bar{q}_{11}=\left.\frac{2 \pi \kappa_{11} r_{w}}{\mu}\left(\frac{\partial \Delta \bar{p}_{11}}{\partial r}\right)\right|_{r=r_{w}}=-\frac{2 \pi \kappa_{11} r_{w}}{\mu}\left(\sigma_{11}\left(A_{1}^{1} K_{1}\left(\sigma_{11}\right)-B_{1}^{1} I_{1}\left(\sigma_{11}\right)\right)\right)
$$

And,

$$
\bar{q}_{21}=\left.\frac{2 \pi \kappa_{21} r_{w}}{\mu}\left(\frac{\partial \Delta \bar{p}_{21}}{\partial r}\right)\right|_{r=r_{w}}=-\frac{2 \pi \kappa_{21} r_{w}}{\mu}\left(\sigma_{21}\left(A_{2}^{1} K_{1}\left(\sigma_{21}\right)-B_{2}^{1} I_{1}\left(\sigma_{21}\right)\right)\right)
$$


Then, replacing (3.1.54) and (3.1.55) in the flow rate CCL:

$$
\sum_{j=1}^{2} \sigma_{j 1} \kappa_{j 1}\left(-A_{j}^{1} K_{1}\left(\sigma_{j 1} r_{w}\right)+B_{j}^{1} I_{1}\left(\sigma_{j 1} r_{w}\right)\right)=-\frac{q \mu}{2 \pi r_{w} u}
$$

Using relations (3.1.50), (3.1.52), (3.1.53) and (3.1.56) the coefficients $A_{j}^{1}, B_{j}^{1}$ and $A_{j}^{2}$ can be obtained by solving a linear system, which is:

$$
\left[\begin{array}{c}
A_{1}^{1} \\
B_{1}^{1} \\
A_{1}^{2} \\
A_{2}^{1} \\
B_{2}^{1} \\
A_{2}^{2}
\end{array}\right]=M^{-1}\left[\begin{array}{c}
-q \mu /\left(2 \pi r_{w} u\right) \\
0 \\
0 \\
0 \\
0 \\
0
\end{array}\right]
$$

Where $M$ in (3.1.57) is given by:

$$
M(1: 6,1: 3)=\left[\begin{array}{ccc}
\kappa_{11} \sigma_{11} K_{1}\left(r_{w} \sigma_{11}\right) & -\kappa_{11} \sigma_{11} I_{1}\left(r_{w} \sigma_{11}\right) & 0 \\
K_{0}\left(r_{w} \sigma_{11}\right) & I_{0}\left(r_{w} \sigma_{11}\right) & 0 \\
K_{1}\left(r_{11} \sigma_{11}\right) & -I_{1}\left(r_{11} \sigma_{11}\right) & -\frac{\kappa_{12} \sigma_{12}}{\kappa_{11} \sigma_{11}} K_{1}\left(r_{11} \sigma_{12}\right) \\
K_{0}\left(r_{11} \sigma_{11}\right) & I_{0}\left(r_{11} \sigma_{11}\right) & -K_{0}\left(r_{11} \sigma_{12}\right) \\
0 & 0 & 0 \\
0 & 0 & 0
\end{array}\right]
$$




$$
M(1: 6,4: 6)=\left[\begin{array}{ccc}
\kappa_{21} \sigma_{21} K_{1}\left(r_{w} \sigma_{21}\right) & -\kappa_{21} \sigma_{21} I_{1}\left(r_{w} \sigma_{21}\right) & 0 \\
-K_{0}\left(r_{w} \sigma_{21}\right) & -I_{0}\left(r_{w} \sigma_{21}\right) & 0 \\
0 & 0 & 0 \\
0 & 0 & 0 \\
K_{1}\left(r_{21} \sigma_{21}\right) & -I_{1}\left(r_{21} \sigma_{21}\right) & -\frac{\kappa_{22} \sigma_{22}}{\kappa_{21} \sigma_{21}} K_{1}\left(r_{21} \sigma_{22}\right) \\
K_{0}\left(r_{21} \sigma_{21}\right) & I_{0}\left(r_{21} \sigma_{21}\right) & -K_{0}\left(r_{21} \sigma_{22}\right)
\end{array}\right]
$$

And, finally, it is possible to calculate the pressure variation at the wellbore:

$$
\Delta \bar{p}_{11}=A_{1}^{1} K_{0}\left(\sigma_{11} r_{w}\right)+B_{1}^{1} I_{0}\left(\sigma_{11} r_{w}\right)
$$

\section{2}

\section{Multilayered Model with Two Regions of Equal Radii per Layer}

Consider now, that there are $n$ layers with 2 regions of radii $r_{j 1}$ and $r_{j 2}$ such that, $r_{j 1}$ and $r_{j 2}$ have the same value for every $j$. The mathematical formulation for this case is similar to the case of two layers considered in the previous section. The flow in each region of each layer $j=2, \ldots, n-1$ in the Laplace field is governed by equation (3.2.1):

$$
\kappa_{j i} \nabla^{2} \Delta \bar{p}_{j i}=\omega_{j} c_{t} u \mu \Delta \bar{p}_{j i}+\left(\Delta \bar{p}_{j i}-\Delta \bar{p}_{(j-1) i}\right) X_{j-1}^{i}-\left(\Delta \bar{p}_{(j+1) i}-\Delta \bar{p}_{j i}\right) X_{j}^{i}
$$

When $j=1$, the PDE is:

$$
\kappa_{j i} \nabla^{2} \Delta \bar{p}_{j i}=\omega_{j} c_{t} u \mu \Delta \bar{p}_{j i}-\left(\Delta \bar{p}_{(j+1) i}-\Delta \bar{p}_{j i}\right) X_{j}^{i}
$$

And for $j=n$, the PDE is: 


$$
\kappa_{j i} \nabla^{2} \Delta \bar{p}_{j i}=\Delta \bar{p}_{j i}=\omega_{j} c_{t} u \mu \Delta \bar{p}_{j i}+\left(\Delta \bar{p}_{j i}-\Delta \bar{p}_{(j-1) i}\right) X_{j-1}^{i}
$$

For $i=1,2$.

The general pressure solution in the Laplace field for this case are given by:

Region 1:

$$
\Delta \bar{p}_{j 1}=A_{j}^{1} K_{0}\left(\sigma_{j 1} r\right)+B_{j}^{1} I_{0}\left(\sigma_{j 1} r\right)
$$

Region 2:

$$
\Delta \bar{p}_{j 2}=A_{j}^{2} K_{0}\left(\sigma_{j 2} r\right)
$$

For $j=1,2, \ldots, n$.

Notice that there are $3 n$ coefficients to be determined now. In order to calculate the pressure solution, the roots $\sigma_{j i}$ also needs to be calculated.

Calculating the laplacian of equations (3.2.4) and (3.2.5) and replacing them in the PDE's, the $2 n$ equations below are given:

Layer 1:

$$
\left(\kappa_{1 i} \sigma_{1 i}^{2}-\omega_{1} c_{t} \mu u-X_{1}^{i}\right) \Delta \bar{p}_{1 i}+X_{1}^{i} \Delta \bar{p}_{2 i}=0
$$

Layer 2:

$$
\left(\kappa_{2 i} \sigma_{2 i}^{2}-\omega_{2} c_{t} \mu u-X_{1}^{i}-X_{2}^{i}\right) \Delta \bar{p}_{2 i}+X_{1}^{i} \Delta \bar{p}_{1 i}+X_{2}^{i} \Delta \bar{p}_{3 i}=0
$$

Layer 3:

$$
\left(\kappa_{3 i} \sigma_{3 i}^{2}-\omega_{3} c_{t} \mu u-X_{2}^{i}-X_{3}^{i}\right) \Delta \bar{p}_{3 i}+X_{2}^{i} \Delta \bar{p}_{2 i}+X_{3}^{i} \Delta \bar{p}_{4 i}=0
$$


Layer n:

$$
\left(\kappa_{n i} \sigma_{n i}^{2}-\omega_{n} c_{t} \mu u-X_{n-1}^{i}-X_{n}^{i}\right) \Delta \bar{p}_{n i}+X_{n-1}^{i} \Delta \bar{p}_{(n-1) i}=0
$$

For each region there are $n$ equations which form a homogeneous tridiagonal-like system, where the non-trivial solution for the pressure variation vector is wanted, hence, like in section 3.1, the determinant of the matrix of this homogeneous system must vanish, and the values of $\sigma_{j i}$ that satisfy that are used.

Now, in order to find the pressure coefficients, equations (3.2.4) and (3.2.5) are used in the coupling conditions, which are given by:

$$
C C L=\left\{\begin{array}{l}
\frac{q \mu}{2 \pi u r_{w}}=\left.\sum_{j=1}^{n}\left(\frac{\partial \Delta \bar{p}_{j 1}}{\partial r}\right)\right|_{r=r_{w}} \\
\Delta \bar{p}_{11}\left(r=r_{w}, t\right)=\Delta \bar{p}_{21}\left(r=r_{w}, t\right) \\
\Delta \bar{p}_{21}\left(r=r_{w}, t\right)=\Delta \bar{p}_{31}\left(r=r_{w}, t\right) \\
\vdots \\
\Delta \bar{p}_{(n-1) 1}\left(r=r_{w}, t\right)=\Delta \bar{p}_{n 1}\left(r=r_{w}, t\right)
\end{array}\right.
$$

$$
C C R=\left\{\begin{array}{l}
\Delta \bar{p}_{11}\left(r=r_{11}, t\right)=\Delta \bar{p}_{12}\left(r=r_{11}, t\right) \\
\Delta \bar{p}_{21}\left(r=r_{21}, t\right)=\Delta \bar{p}_{22}\left(r=r_{21}, t\right) \\
\vdots \\
\Delta \bar{p}_{n 1}\left(r=r_{n 1}, t\right)=\Delta \bar{p}_{n 2}\left(r=r_{n 1}, t\right) \\
\left.\left(r \frac{\partial \Delta \bar{p}_{11}}{\partial r}\right)\right|_{r=r_{11}}=\left.\frac{k_{12}}{k_{11}}\left(r \frac{\partial \Delta \bar{p}_{12}}{\partial r}\right)\right|_{r=r_{11}} \\
\left.\left(r \frac{\partial \Delta \bar{p}_{21}}{\partial r}\right)\right|_{r=r_{21}}=\left.\frac{k_{22}}{k_{21}}\left(r \frac{\partial \Delta \bar{p}_{22}}{\partial r}\right)\right|_{r=r_{21}} \\
\vdots \\
\left.\left(r \frac{\partial \Delta \bar{p}_{n 1}}{\partial r}\right)\right|_{r=r_{n 1}}=\left.\frac{k_{n 2}}{k_{n 1}}\left(r \frac{\partial \Delta \bar{p}_{n 2}}{\partial r}\right)\right|_{r=r_{n 1}}
\end{array}\right.
$$


Now, with the $3 n$ equations above, the following linear system can be assembled to find the coefficients:

$$
\left[\begin{array}{c}
A_{1}^{1} \\
B_{1}^{1} \\
A_{1}^{2} \\
A_{2}^{1} \\
B_{2}^{1} \\
A_{2}^{2} \\
\vdots \\
A_{n}^{1} \\
B_{n}^{1} \\
A_{n}^{2}
\end{array}\right]=M^{-1}\left[\begin{array}{c}
-q \mu /\left(2 \pi r_{w} u\right) \\
0 \\
0 \\
0 \\
0 \\
0 \\
\vdots \\
0 \\
0 \\
0
\end{array}\right]
$$

Where the matrix $\mathrm{M}$ mentioned above is defined from the $3 n$ equations (3.2.10) and (3.2.11). Then, it is possible to calculate the pressure variation at the wellbore:

$$
\Delta \bar{p}_{11}=A_{1}^{1} K_{0}\left(\sigma_{11} r_{w}\right)+B_{1}^{1} I_{0}\left(\sigma_{11} r_{w}\right)
$$

\section{3}

\section{Two Layered Model with Two Regions of Distinct Radii per Layer}

In this section, the following case is considered, there are two layers with two regions of distinct radii. In that case, there is a region that connects different regions of permeability of layers 1 and 2 and this scenario comes down to the case of 3 regions with equal radii, where the region 2 is created artificially.

Figure 3.2 illustrates this model: 


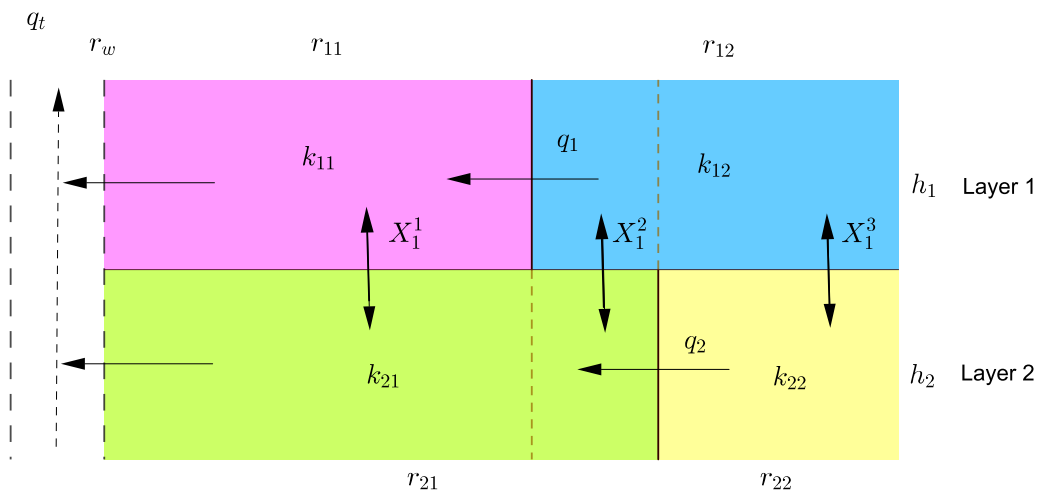

Figure 3.2: Reservoir with two layers and two regions of different radii

For this case, there are 3 different semipermeability coefficients, $X_{1}^{1}, X_{1}^{2}$ and $X_{1}^{3}$. In figure 3.2 , the coefficient $X_{1}^{2}$ connects region 2 in layer 1 with region 1 in layer 2. $X_{1}^{2}$ is given by:

$$
X_{1}^{2}=\frac{2}{2\left[\left(\Delta h_{1}\right) / k_{v 1}^{2}\right]+\chi_{2}^{2}+\chi_{1}^{2}}
$$

With,

$$
\chi_{1}^{2}=\frac{h_{1}}{k_{z 1}^{2}}
$$

The vertical permeability considered for layer 1 is the one in region 2, and for layer 2 , is the vertical permeability of region 1 :

$$
\chi_{2}^{2}=\frac{h_{2}}{k_{z 2}^{1}}
$$

Unlike $X_{1}^{1}$ and $X_{1}^{3}$ which relates the same region in the layers. In the Laplace field, the above problem is given by: 


\section{Layer 1:}

Region 1:

$$
\begin{aligned}
& P D E: \quad \kappa_{11} \nabla^{2} \Delta \bar{p}_{11}=\omega_{1} c_{t} \mu u \Delta \bar{p}_{11}-X_{1}^{1}\left(\Delta \bar{p}_{21}-\Delta \bar{p}_{11}\right) \\
& I C: \quad \Delta \bar{p}_{11}(r, 0)=0 \\
& I B C: \quad \bar{q}_{11}=\left.\frac{2 \pi \kappa_{11} r}{\mu}\left(\frac{\partial \Delta \bar{p}_{11}}{\partial r}\right)\right|_{r=r_{w}}
\end{aligned}
$$

Region 2:

$$
\begin{aligned}
& \text { PDE : } \kappa_{12} \nabla^{2} \Delta \bar{p}_{12}=\omega_{1} c_{t} \mu u \Delta \bar{p}_{12}-X_{1}^{2}\left(\Delta \bar{p}_{22}-\Delta \bar{p}_{12}\right) \\
& I C: \Delta \bar{p}_{12}(r, 0)=0
\end{aligned}
$$

Region 3:

$$
\begin{aligned}
& \text { PDE }: \quad \kappa_{13} \nabla^{2} \Delta \bar{p}_{13}=\omega_{1} c_{t} \mu u \bar{p}_{13}-X_{1}^{3}\left(\Delta \bar{p}_{23}-\Delta \bar{p}_{13}\right) \\
& I C: \Delta \bar{p}_{13}(r, 0)=0 \\
& E B C: \lim _{r \rightarrow \infty} \Delta \bar{p}_{13}(r, t)=0
\end{aligned}
$$

The coupling conditions for the regions (CCR) for layer 1 is given by:

$$
\left\{\begin{array}{c}
\Delta \bar{p}_{11}\left(r=r_{11}, t\right)=\Delta \bar{p}_{12}\left(r=r_{11}, t\right) \\
\Delta \bar{p}_{12}\left(r=r_{12}, t\right)=\Delta \bar{p}_{13}\left(r=r_{12}, t\right) \\
\left.\left(r \frac{\partial \Delta \bar{p}_{11}}{\partial r}\right)\right|_{r=r_{11}}=\left.\frac{k_{12}}{k_{11}}\left(r \frac{\partial \Delta \bar{p}_{12}}{\partial r}\right)\right|_{r=r_{11}} \\
\left.\left(r \frac{\partial \Delta \bar{p}_{12}}{\partial r}\right)\right|_{r=r_{12}}=\left.\frac{k_{13}}{k_{12}}\left(r \frac{\partial \Delta \bar{p}_{13}}{\partial r}\right)\right|_{r=r_{12}}
\end{array}\right.
$$




\section{Layer 2:}

Region 1:

$$
\begin{aligned}
& \text { PDE : } \quad \kappa_{21} \nabla^{2} \Delta \bar{p}_{21}=\omega_{2} c_{t} \mu u \Delta \bar{p}_{21}+X_{1}^{1}\left(\Delta \bar{p}_{21}-\Delta \bar{p}_{11}\right) \\
& I C: \quad \Delta \bar{p}_{21}(r, t=0)=0 \\
& I B C: \quad \bar{q}_{21}=\left.\frac{2 \pi \kappa_{21} r}{\mu}\left(\frac{\partial \Delta \bar{p}_{21}}{\partial r}\right)\right|_{r=r_{w}}
\end{aligned}
$$

Region 2:

$$
\begin{aligned}
& \text { PDE : } \kappa_{22} \nabla^{2} \Delta \bar{p}_{22}=\omega_{2} c_{t} \mu u \Delta \bar{p}_{22}+X_{1}^{2}\left(\Delta \bar{p}_{22}-\Delta \bar{p}_{12}\right) \\
& I C: \Delta \bar{p}_{22}(r, t=0)=0
\end{aligned}
$$

Region 3:

$$
\begin{aligned}
& \text { PDE : } \kappa_{23} \nabla^{2} \Delta \bar{p}_{23}=\omega_{2} c_{t} \mu u \Delta \bar{p}_{23}+X_{1}^{3}\left(\Delta \bar{p}_{23}-\Delta \bar{p}_{13}\right) \\
& I C: \Delta \bar{p}_{23}(r, 0)=0 \\
& E B C: \lim _{r \rightarrow \infty} \Delta \bar{p}_{23}(r, t)=0
\end{aligned}
$$

The CCR for layer 2 is given by:

$$
\left\{\begin{array}{c}
\Delta \bar{p}_{21}\left(r=r_{21}, t\right)=\Delta \bar{p}_{22}\left(r=r_{21}, t\right) \\
\Delta \bar{p}_{22}\left(r=r_{22}, t\right)=\Delta \bar{p}_{23}\left(r=r_{22}, t\right) \\
\left.\left(r \frac{\partial \Delta \bar{p}_{21}}{\partial r}\right)\right|_{r=r_{21}}=\left.\frac{k_{22}}{k_{21}}\left(r \frac{\partial \Delta \bar{p}_{22}}{\partial r}\right)\right|_{r=r_{21}} \\
\left.\left(r \frac{\partial \Delta \bar{p}_{22}}{\partial r}\right)\right|_{r=r_{22}}=\left.\frac{k_{23}}{k_{22}}\left(r \frac{\partial \Delta \bar{p}_{23}}{\partial r}\right)\right|_{r=r_{22}}
\end{array}\right.
$$

The coupling condition between the layers (CCL) is given by: 


$$
\left\{\begin{array}{c}
\Delta \bar{p}_{11}\left(r=r_{w}, t\right)=\Delta \bar{p}_{21}\left(r=r_{w}, t\right) \\
\frac{q \mu}{2 \pi u r_{w}}=\left.\frac{\partial \Delta \bar{p}_{11}}{\partial r}\right|_{r=r_{w}}+\left.\frac{\partial \Delta \bar{p}_{21}}{\partial r}\right|_{r=r_{w}}
\end{array}\right.
$$

The general pressure solution for the 3 regions are given by:

Region 1:

$$
\Delta \bar{p}_{j 1}=A_{j}^{1} K_{0}\left(r \sigma_{j 1}\right)+B_{j}^{1} I_{0}\left(r \sigma_{j 1}\right)
$$

Region 2:

$$
\Delta \bar{p}_{j 2}=A_{j}^{2} K_{0}\left(r \sigma_{j 2}\right)+B_{j}^{2} I_{0}\left(r \sigma_{j 2}\right)
$$

Region 3:

$$
\Delta \bar{p}_{j 3}=A_{j}^{3} K_{0}\left(r \sigma_{j 3}\right)
$$

There are 10 pressure coefficients to be determined. To calculate the pressure variation, it is enough to calculate the roots $\sigma_{j i}$ and these 10 coefficients. First, to find the $\sigma_{j i}$ the following equations for each region, obtained like in the previous sections, are used:

Layer 1:

$$
\left(\kappa_{1 i} \sigma_{1 i}^{2}-\omega_{1} c_{t} \mu u-X_{1}^{i}\right) \Delta \bar{p}_{1 i}+X_{1}^{i} \Delta \bar{p}_{2 i}=0
$$

Layer 2:

$$
\left(\kappa_{2 i} \sigma_{2 i}^{2}-\omega_{2} c_{t} \mu u-X_{1}^{i}\right) \Delta \bar{p}_{2 i}+X_{1}^{i} \Delta \bar{p}_{1 i}=0
$$


With (3.3.26) and (3.3.27), the following linear system is assembled for each region $i=1,2,3$ :

$$
\left[\begin{array}{cc}
\kappa_{1 i} \sigma_{1 i}^{2}-\omega_{1} c_{t} \mu u-X_{1}^{i} & X_{1}^{i} \\
X_{1}^{i} & \kappa_{2 i} \sigma_{2 i}^{2}-\omega_{2} c_{t} \mu u-X_{1}^{i}
\end{array}\right]\left[\begin{array}{c}
\Delta \bar{p}_{1 i} \\
\Delta \bar{p}_{2 i}
\end{array}\right]=0
$$

The values of $\sigma_{j i}$ are found as the determinant of the matrix in (3.3.28) must vanish.

Now, it is left to find the values of the pressure coefficients. The coupling conditions of the regions and of the layers (CCR and CCL), provide 10 equations:

Flow rate CCL:

$$
\sum_{j=1}^{2} \sigma_{j 1} \kappa_{j 1}\left(-A_{j}^{1} K_{1}\left(\sigma_{j 1} r_{w}\right)+B_{j}^{1} I_{1}\left(\sigma_{j 1} r_{w}\right)\right)=-\frac{q \mu}{2 \pi r_{w} u}
$$

Pressure CCL:

$$
A_{1}^{1} K_{0}\left(r_{w} \sigma_{11}\right)+B_{1}^{1} I_{0}\left(r_{w} \sigma_{11}\right)=A_{2}^{1} K_{0}\left(r_{w} \sigma_{21}\right)+B_{2}^{1} I_{0}\left(r_{w} \sigma_{21}\right)
$$

First pressure CCR:

$A_{j}^{1} K_{0}\left(r_{j 1} \sigma_{j 1}\right)+B_{j}^{1} I_{0}\left(r_{j 1} \sigma_{j 1}\right)=A_{j}^{2} K_{0}\left(r_{j 1} \sigma_{j 2}\right)+B_{j}^{2} I_{0}\left(r_{j 1} \sigma_{j 2}\right)$

For $j=1,2$.

Second pressure CCR:

$$
A_{j}^{2} K_{0}\left(r_{j 2} \sigma_{j 2}\right)+B_{j}^{2} I_{0}\left(r_{j 2} \sigma_{j 2}\right)=A_{j}^{3} K_{0}\left(r_{j 2} \sigma_{j 3}\right)
$$

For $j=1,2$. 
First flow rate CCR:

$$
A_{j}^{1} K_{1}\left(r_{j 1} \sigma_{j 1}\right)-B_{j}^{1} I_{1}\left(r_{j 1} \sigma_{j 1}\right)=\frac{\kappa_{j 2} \sigma_{j 2}}{\kappa_{j 1} \sigma_{j 1}}\left(A_{j}^{2} K_{1}\left(r_{j 1} \sigma_{j 2}\right)-B_{j}^{2} I_{1}\left(r_{j 1} \sigma_{j 2}\right)\right)
$$

For $j=1,2$.

Second flow rate CCR:

$$
A_{j}^{1} K_{1}\left(r_{j 1} \sigma_{j 1}\right)-B_{j}^{1} I_{1}\left(r_{j 2} \sigma_{j 2}\right)=\frac{\kappa_{j 3} \sigma_{j 3}}{\kappa_{j 2} \sigma_{j 2}} A_{j}^{3} K_{1}\left(r_{j 2} \sigma_{j 3}\right)
$$

For $j=1,2$.

Using equations (3.3.29) to (3.3.34), the following linear system is solved

\begin{tabular}{|c|c|c|c|c|}
\hline$\kappa_{11} \sigma_{11} K_{1}\left(r_{w} \sigma_{11}\right)$ & $-\kappa_{11} \sigma_{11} I_{1}\left(r_{w} \sigma_{11}\right)$ & 0 & 0 & 0 \\
\hline$K_{0}\left(r_{w} \sigma_{11}\right)$ & $I_{0}\left(r_{w} \sigma_{11}\right)$ & 0 & 0 & 0 \\
\hline$K_{1}\left(r_{11} \sigma_{11}\right)$ & $-I_{1}\left(r_{11} \sigma_{11}\right)$ & $-\frac{\kappa_{12} \sigma_{12}}{\kappa_{11} \sigma_{11}} K_{1}\left(r_{11} \sigma_{12}\right)$ & $\frac{\kappa_{12} \sigma_{12}}{\kappa_{11} \sigma_{11}} I_{1}\left(r_{11} \sigma_{12}\right)$ & 0 \\
\hline 0 & 0 & $K_{1}\left(r_{12} \sigma_{12}\right)$ & $-I_{1}\left(r_{12} \sigma_{12}\right)$ & $-\frac{\kappa_{13} \sigma_{13}}{\kappa_{12} \sigma_{12}} K_{1}\left(r_{12} \sigma_{13}\right)$ \\
\hline$K_{0}\left(r_{11} \sigma_{11}\right)$ & $I_{0}\left(r_{11} \sigma_{11}\right)$ & $-K_{0}\left(r_{11} \sigma_{12}\right)$ & $-I_{0}\left(r_{11} \sigma_{12}\right)$ & 0 \\
\hline 0 & 0 & $K_{0}\left(r_{12} \sigma_{12}\right)$ & $I_{0}\left(r_{12} \sigma_{12}\right)$ & $-K_{0}\left(r_{12} \sigma_{13}\right)$ \\
\hline 0 & 0 & 0 & 0 & 0 \\
\hline 0 & 0 & 0 & 0 & 0 \\
\hline 0 & 0 & 0 & 0 & 0 \\
\hline 0 & 0 & 0 & 0 & 0 \\
\hline
\end{tabular}
to find the pressure coefficients hence the pressure variation at the wellbore: 


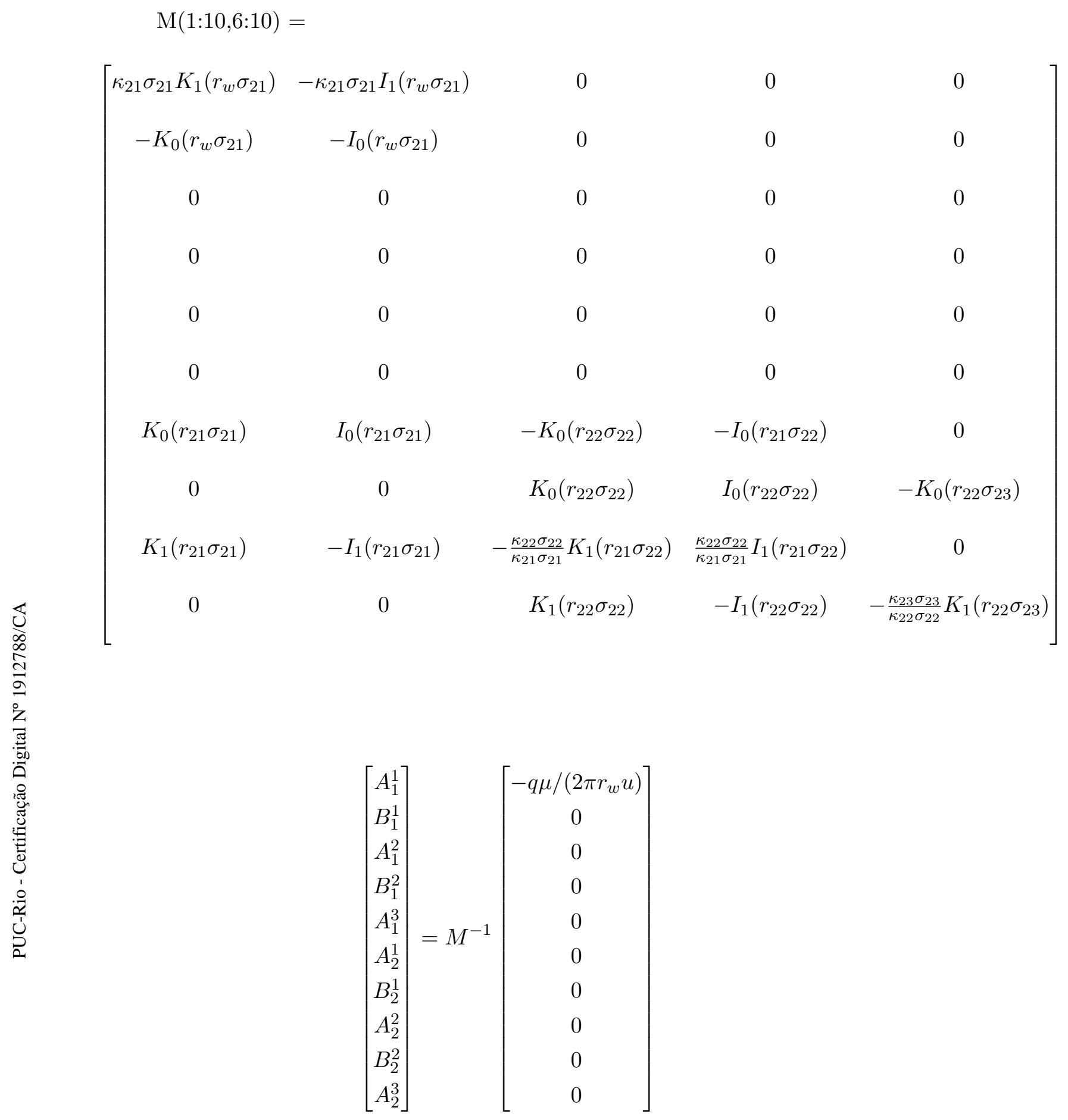

Notice that this deduction also works for a 3 regions of equal radii per layer case.

For the case of a multilayered model with two regions of distinct radii in each layer, it is not always possible to solve it like a 3 regions of equal radii case. For instance, consider a 3 layer reservoir model and that $r_{11} \neq r_{21} \neq r_{31}$, then, extending each interface to all the other layers, like was done in this section, there will not be 1 artificial region in each layer but 2 . That is, this problem would not be a 3 regions 
of equal radii case, but one with 4 regions of equal radii, this example is illustrated in figure 3.3:

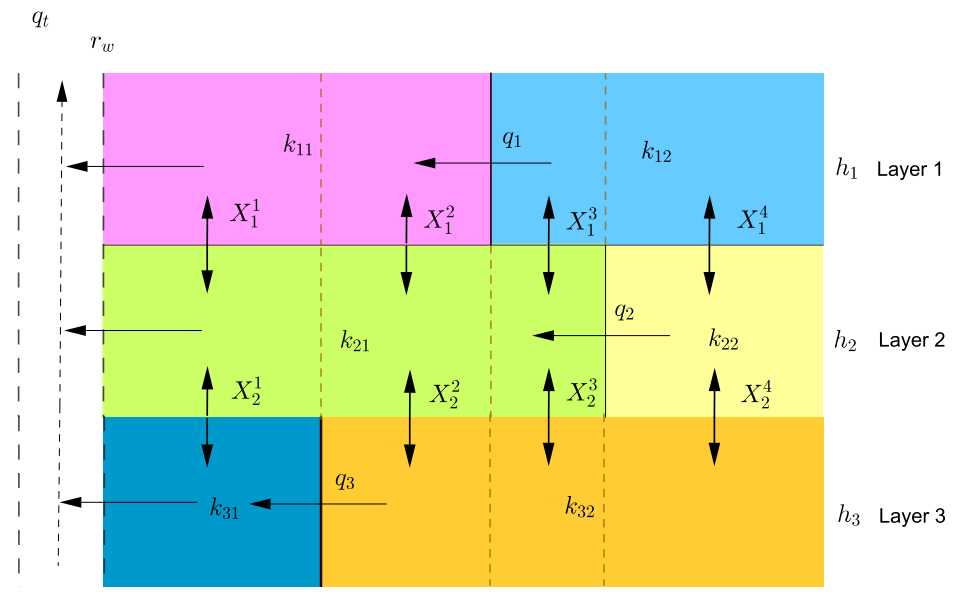

Figure 3.3: 3 layers with 4 regions of equal radii created artificially

This problem, along with other number of regions $(>2)$ of distinct radii per layer, is treated later, in chapter 4 .

\section{4 \\ Formation Damage}

In this section, formation damage is considered. Formation damage, also known as skin effect, is defined as a damaged wellbore or as a region, around the wellbore, with permeability that was reduced, either by a fluid of drilling/completion, swelling of clays, inversion of wettability, among other reasons or with permeability that was improved for some reason, for example due to acidification. Figure 3.4 illustrates a damaged region around the wellbore:

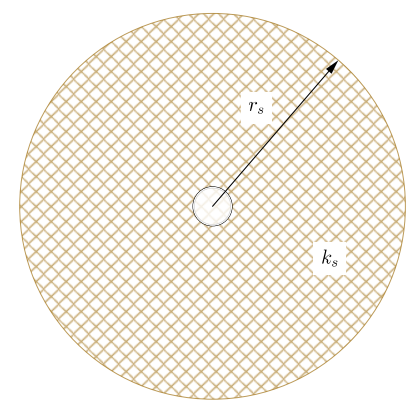

Figure 3.4: Damage region of radius $r_{s}$ and permeability $k_{s}$ 
Usually, the formation damage region's permeability is smaller than the reservoir's permeability. Figure 3.5 illustrates the impact of the skin effect in the pressure curve:

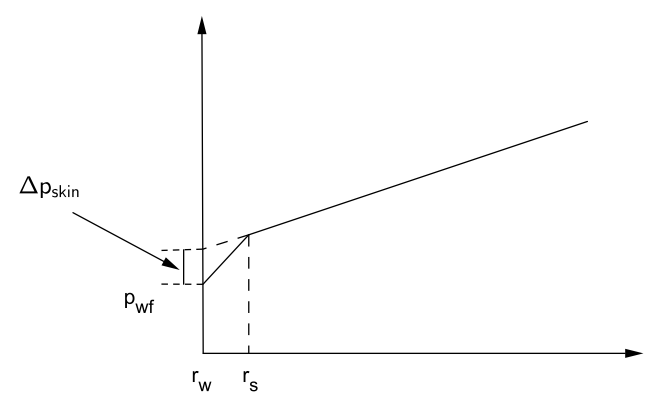

Figure 3.5: Representation of damage factor $\Delta p_{\text {skin }}$

The skin factor, or simply skin, is defined as an additional dimensionless decline on the pressure:

$$
S=\frac{k h}{\alpha_{p} q B \mu} \Delta p_{\text {skin }}
$$

The formation damage region quickly enters on steady state, then $S_{j}$ is given, for each layer $j$, by:

$$
S_{j}=\left(\frac{k_{j}}{k_{s j}}-1\right) \log \left(\frac{r_{s j}}{r_{w}}\right)
$$

This equation is known as the Hawkins formula. For multilayered reservoirs, the skin factor is given by the average of the skin factors for each individual layer, weighted by their permeability-thickness $(\kappa)$ product [9]:

$$
S=\frac{\sum_{j=1}^{n} k_{j} h_{j} S_{j}}{k_{j} h_{j}}
$$

In addition, using equation (3.4.2) it is possible to conclude that: 
$\left\{\begin{array}{l}\text { For } k=k_{s}, S=0 \text { that is, there is no damage in that layer } \\ \text { For } k>k_{s}, S>0 \text { that is, there is damage in that layer } \\ \text { For } k<k_{s}, S<0 \text { that is, the well is stimulated }\end{array}\right.$

Now, notice that the solution to the case considering a damaged region around the wellbore is equivalent to the solution considering more then one region of permeability in each layer. That is why the deduction made in the previous sections allows to extend to the case considering the skin factor. Figure 3.6 shows a two layered reservoir with a damaged region near the wellbore:

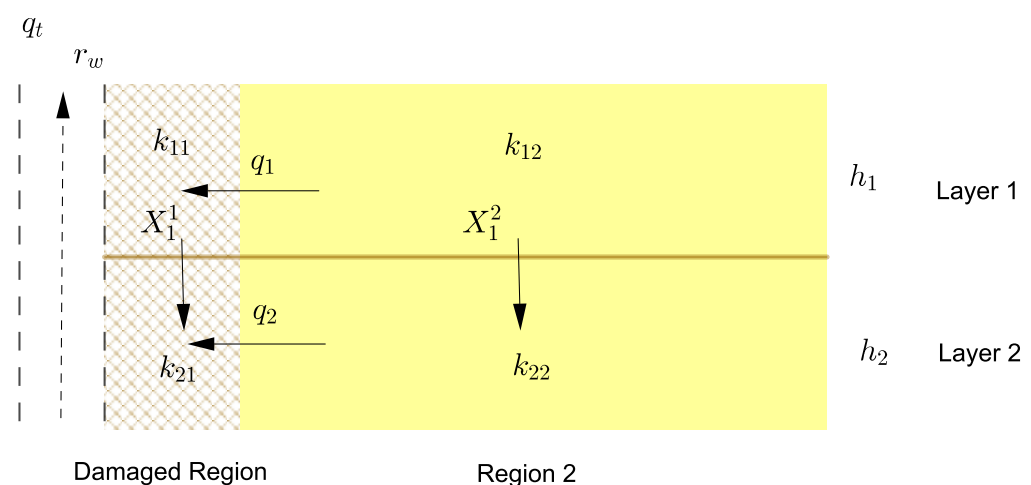

Figure 3.6: Two layered reservoir with damaged region near the wellbore

The solution for this case relies on the equal radii studied in section 3.1. Likewise, if the radii of the damaged region are different from layer 1 to layer 2 the case will rely on the one studied in section 3.3. 


\section{Pressure Response Considering Formation Crossflow in Mul- tilayered Reservoirs Under Single Phase Flow with Multiple Regions per Layer}

Consider the general case now, there are $n$ layers in the reservoir and there are $m$ regions for each layer $j$. These regions can either have equal or different radii. In this chapter, an analytical model is presented to find the pressure response for this much general case.

\section{1}

\section{Model Description}

The number of regions in the layers may be different, and it will be represented by $m_{j}$ for layer $j$. It is important to add that even if the numbers of regions are the same for two connected layers, i.e, $m_{j}=m_{j+1}$, the radii of these regions can be different. These two situations must be considered and, to illustrate them better, without loss of generality, a two layer reservoir is considered.

The number of regions in layer 1 is a finite natural number $m_{1}$ and, likewise, the number of regions in layer 2 is $m_{2}$. The radius of each regions for layer 1 can be represented: $r(1,1), r(1,2), r(1,3) \ldots r\left(1, m_{1}\right)$ and for layer 2 : $r(2,1), r(2,2), r(2,3) \ldots r\left(2, m_{2}\right)$. The first situation considered is if $r(1, l)=r(2, k)$ for some $l, k$. The second one is when $r(1, i) \neq r(2, k)$ for all $i, k$. For the first case, the number of $X_{j}^{i}$ coefficients is $m_{1}+m_{2}-1-s$, where $s$ is the number of incidents where $r(1, l)=r(2, k)$. For the second case, which is a particular case of the first one, the number of $X_{j}^{i}$ coefficients is $m_{1}+m_{2}-1$. This affirmation is demonstrated in Appendix C.

For example, in figure 4.1, the first two layers represented have, respectively, 3 and 4 regions. The radius of region 2 in layer 1 and the radius of region 3 in layer 2 are the only values that coincide $(r(1,2)=r(2,3))$. Then, $m_{1}=3, m_{2}=4$ and $s=1$. Hence, there are $3+4-1-1=5$ semipermeability coefficients relating these layers. 


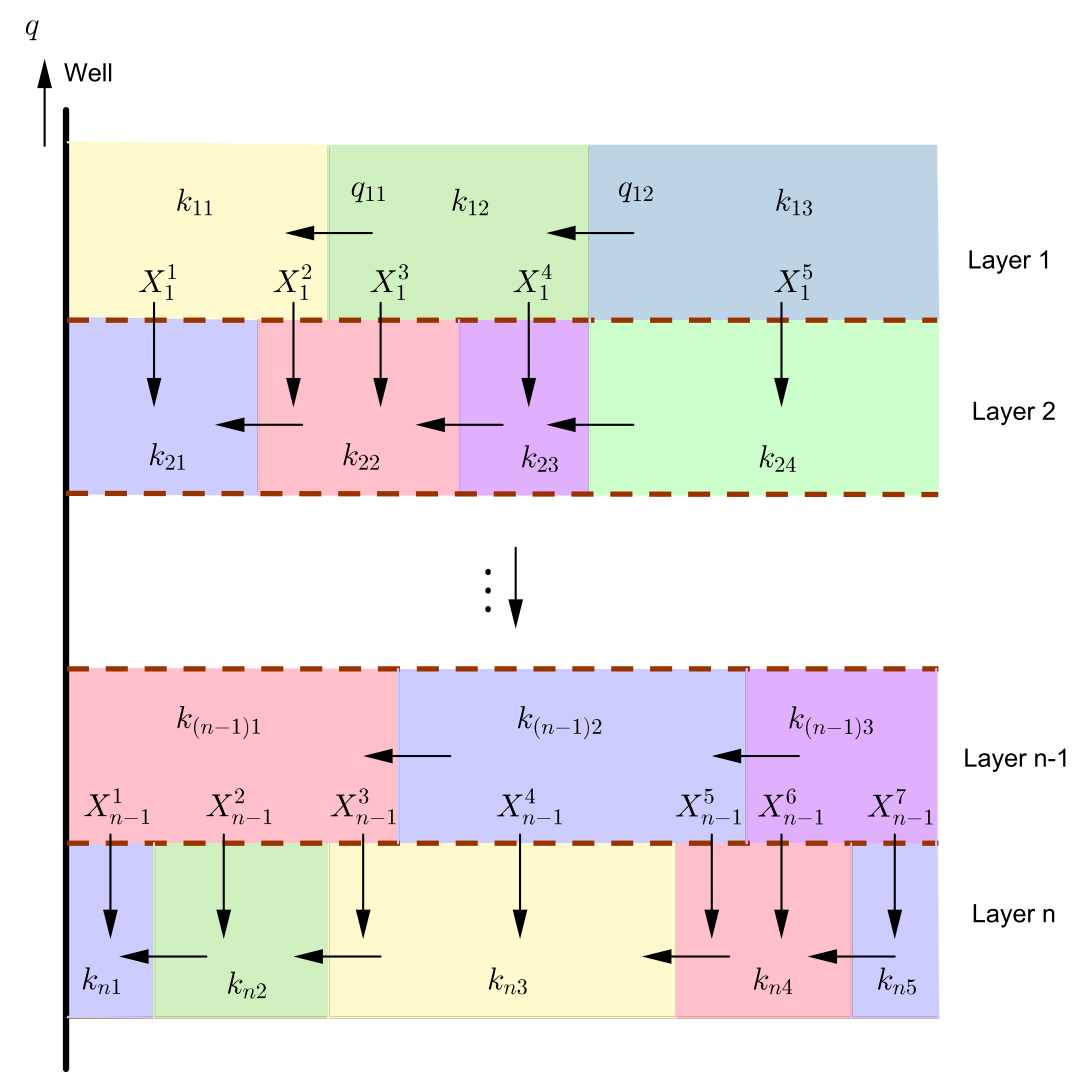

Figure 4.1: Reservoir with $n$ connected layers and many regions

Consider the following number:

$$
\alpha=\left(\sum_{j=1}^{n}\left(m_{j}-1\right)\right)-s
$$

Then, extending the existent interfaces in each layer and creating the artificial regions similarly as in section 3.3 , illustrated in figure 4.2 , this problem can be treated as one with $\alpha$ regions of equal radii. 


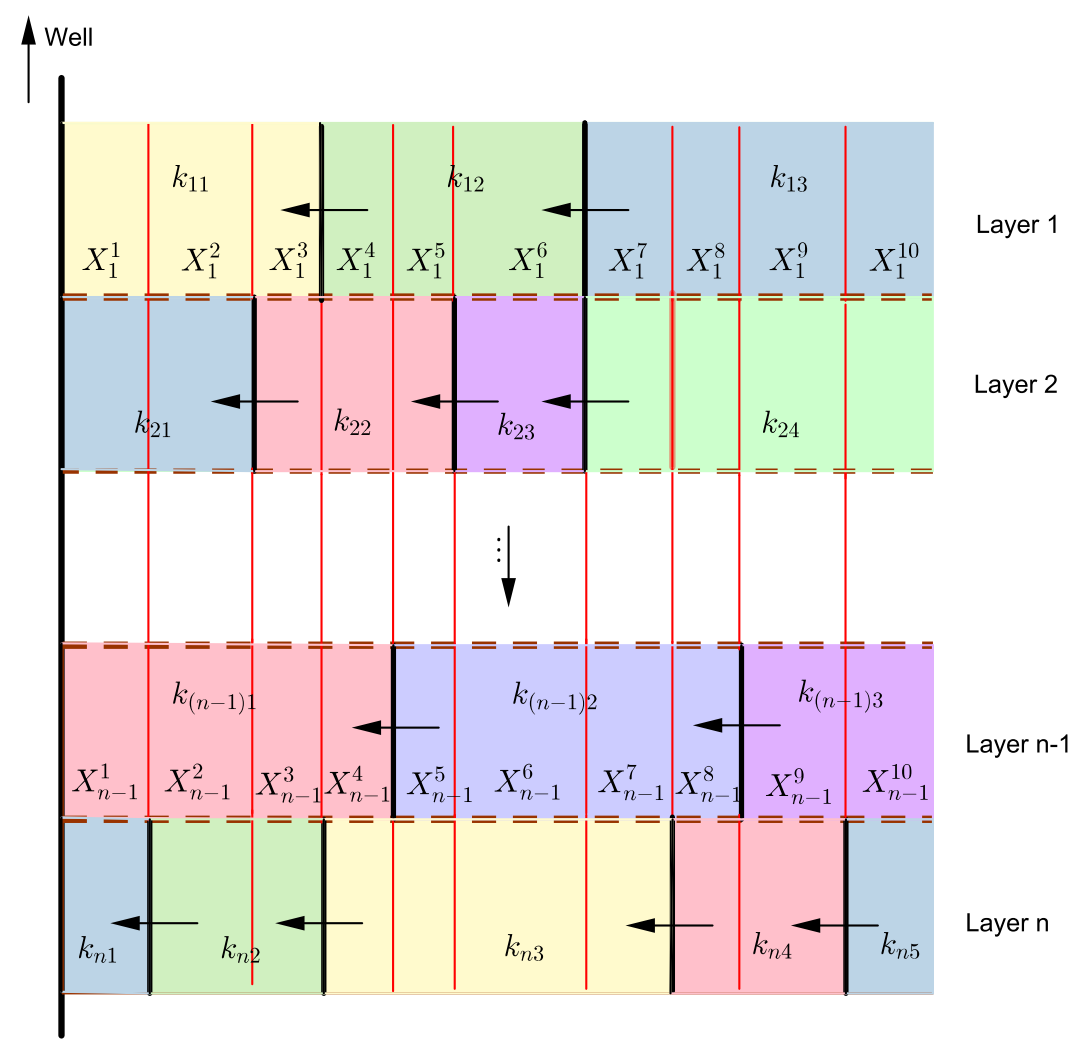

Figure 4.2: $\alpha$ extensions of the regions in each layer

It is important to define well each semipermeability coefficient $X_{j}^{i}$ for the $\alpha$ regions, i.e, observing correctly the permeability regions it is relating. After the $\alpha$ extensions of the regions in each layer, there might be regions with the same $X_{j}^{i}$ coefficients, like in figure $4.2, X_{1}^{1}=X_{1}^{2}$.

Notice that there are $m_{j}+m_{j+1}-1-s$ different values of the $X_{j}^{i}$ coefficients and $\alpha-\left(m_{j}+m_{j+1}-1-s\right)$ repeated values of it for each adjacency.

Besides that, the usual assumptions will also be considered; Each layer is considered homogeneous, laterally isotropic, has a single phase flow of viscosity $(\mu)$ that is constant, and also constant small total compressibility $\left(c_{t}\right)$ in all regions. The permeability $\left(k_{j i}\right)$ varies in each layer and also in each region. The porosity $\left(\phi_{j}\right)$ may have a different value in each layer. As well as the thickness $\left(h_{j}\right)$. Constant flow rate at the wellbore is considered. Formation damage at the well or along the reservoir will be disregarded, as well as storage at the well. 
Chapter 4. Pressure Response Considering Formation Crossflow in Multilayered

\section{2}

\section{Mathematical Formulation}

Consider $m=\alpha$. The PDE (4.2.1) model this problem for layers $j=2, \ldots, n-1$ and regions $i=1, \ldots, m$ in layer $j$ in the Laplace field:

$$
\kappa_{j i} \nabla^{2} \Delta \bar{p}_{j i}=\omega_{j} c_{t} u \mu \Delta \bar{p}_{j i}+\left(\Delta \bar{p}_{j i}-\Delta \bar{p}_{(j-1) i}\right) X_{j-1}^{i}-\left(\Delta \bar{p}_{(j+1) i}-\Delta \bar{p}_{j i}\right) X_{j}^{i}
$$

When $j=1$, the PDE is:

$$
\kappa_{j i} \nabla^{2} \Delta \bar{p}_{j i}=\omega_{j} c_{t} u \mu \Delta \bar{p}_{j i}-\left(\Delta \bar{p}_{(j+1) i}-\Delta \bar{p}_{j i}\right) X_{j}^{i}
$$

And for $j=n$, the PDE is:

$$
\kappa_{j i} \nabla^{2} \Delta \bar{p}_{j i}=\omega_{j} c_{t} u \mu \Delta \bar{p}_{j i}+\left(\Delta \bar{p}_{j i}-\Delta \bar{p}_{(j-1) i}\right) X_{j-1}^{i}
$$

For all regions $i$ in layers $j=1, \ldots, n$ the initial condition is given by:

$$
I C: \Delta \bar{p}_{j i}(r, 0)=0
$$

For layers $j=1, \ldots, n$ and region $i=1$ the inner boundary condition is given:

$$
I B C: \bar{q}_{j i}=\left.\frac{2 \pi \kappa_{j i} r u}{\mu}\left(\frac{\partial \Delta \bar{p}_{j i}}{\partial r}\right)\right|_{r=r_{w}}
$$

And for layers $j=1, \ldots, n$ and region $i=m$ there is also an additional condition, the external boundary condition:

$$
E B C: \lim _{r \rightarrow \infty} \Delta \bar{p}_{j i}(r, t)=0
$$

The coupling conditions between regions $i-1$ and $i$ in each layer $j$ are given by pressure and flow rate equality at the interface between the regions. Hence, the CCR is defined below: 


$$
\left\{\begin{array}{l}
\Delta \bar{p}_{j(i-1)}\left(r=r_{j(i-1)}, t\right)=\Delta \bar{p}_{j i}\left(r=r_{j(i-1)}, t\right) \\
\bar{q}_{j(i-1)}=\bar{q}_{j i}
\end{array}\right.
$$

Using Darcy's law it is possible to rewrite the flow rate relation of the CCR:

$$
\left\{\begin{array}{l}
\Delta \bar{p}_{j(i-1)}\left(r=r_{j(i-1)}, t\right)=\Delta \bar{p}_{j i}\left(r=r_{j(i-1)}, t\right) \\
\left.\left(r \frac{\partial \Delta \bar{p}_{j(i-1)}}{\partial r}\right)\right|_{r=r_{j(i-1)}}=\left.\frac{\kappa_{j i}}{\kappa_{j(i-1)}}\left(r \frac{\partial \Delta \bar{p}_{j i}}{\partial r}\right)\right|_{r=r_{j(i-1)}}
\end{array}\right.
$$

Where $j=1, \ldots, n$ and $i=2, \ldots, m$.

The coupling condition between layers (CCL) is obtained considering that the pressure drop is equal in the layers at the well and that the flow rate at the well is given by the sum of the flow rates of each layer, that is:

$$
\left\{\begin{array}{l}
\Delta \bar{p}_{(j-1) 1}\left(r=r_{w}, t\right)=\Delta \bar{p}_{j 1}\left(r=r_{w}, t\right) \\
\frac{q}{u}=\bar{q}_{11}+\bar{q}_{21}+\ldots+\bar{q}_{n 1}
\end{array}\right.
$$

For $j=2, \ldots, n$. Using Darcy's law in the flow rate CCL as well, equation (4.2.9) can be rewritten:

$$
C C L:\left\{\begin{array}{l}
\Delta \bar{p}_{(j-1) 1}\left(r=r_{w}, t\right)=\Delta \bar{p}_{j 1}\left(r=r_{w}, t\right) \\
\frac{q \mu}{2 \pi u \sum_{j=1}^{n} \kappa_{j 1}}=\left.\sum_{j=1}^{n}\left(r \frac{\partial \Delta \bar{p}_{j 1}}{\partial r}\right)\right|_{r=r_{w}}
\end{array}\right.
$$

For $j=2, \ldots, n$.

The general pressure solution in the Laplace field is well known using the Bessel functions. They are described below for each region $i$ in layer $j$ :

Regions $i=1, \ldots, m-1$ :

$$
\Delta \bar{p}_{j i}=A_{j}^{i} K_{0}\left(r \sigma_{j i}\right)+B_{j}^{i} I_{0}\left(r \sigma_{j i}\right)
$$




$$
\Delta \bar{p}_{j i}=A_{j}^{i} K_{0}\left(r \sigma_{j i}\right)
$$

Using the solutions above, it can be seen that $\nabla^{2} \Delta \bar{p}_{j i}=\sigma_{j i}^{2} \Delta \bar{p}_{j i}$. Hence, replacing this relation in the PDE:

$\left(\kappa_{j i} \sigma_{j i}^{2}-\omega_{j} c_{t} \mu u\right) \Delta \bar{p}_{j i}-\left(\Delta \bar{p}_{j i}-\Delta \bar{p}_{(j-1) i}\right) X_{j-1}^{i}+\left(\Delta \bar{p}_{(j+1) i}-\Delta \bar{p}_{j i}\right) X_{j}^{i}=0$

Rearranging equation (4.2.13):

$$
\left(\kappa_{j i} \sigma_{j i}^{2}-\omega_{j} c_{t} \mu u-X_{j-1}^{i}-X_{j}^{i}\right) \Delta \bar{p}_{j i}+X_{j-1}^{i} \Delta \bar{p}_{(j-1) i}+X_{j}^{i} \Delta \bar{p}_{(j+1) i}=0
$$

This is a homogeneous linear system for each region $i$ where the nontrivial solution is wanted, that is, $\Delta \bar{p}_{j i} \neq 0$. That is true only if each matrix below is singular, and that implies that its determinant must vanish:

$$
a_{j k}^{i}= \begin{cases}X_{j-1}^{i}, & \text { for } k=j-1 ; j>1, \\ \kappa_{j i} \sigma^{2}-\omega_{j} c_{t} \mu u-X_{j-1}^{i}-X_{j}^{i}, & \text { for } k=j, \\ X_{j}^{i}, & \text { for } k=j+1 ; j<n, \\ 0, & \text { for } k \neq j-1, j, \text { or } j+1 .\end{cases}
$$

For $i=1, \ldots, m$.

To find the values of $\sigma_{j i}$ by vanishing the determinant of the matrices above is, as explained in chapter 3 , equivalent to finding the eigenvalues of each matrix below, dividing them by $\kappa_{j i}$ and then extracting its root:

$$
b_{j k}^{i}= \begin{cases}-X_{j-1}^{i}, & \text { for } k=j-1 ; j>1, \\ \omega_{j} c_{t} \mu u+X_{j-1}^{i}+X_{j}^{i}, & \text { for } k=j, \\ -X_{j}^{i}, & \text { for } k=j+1 ; j<n, \\ 0, & \text { for } k \neq j-1, j, \text { or } j+1 .\end{cases}
$$

For $i=1, \ldots, m$. 
Now, with the values of $\sigma_{j i}$ calculated, the coefficients of the pressure solution must be found. It can be seen in equations (4.2.11) and (4.2.12), that there are $(2 m-1) n$ coefficients. The relations provided by the coupling conditions between layers and regions are, precisely, that many. The first $n$ relations are provided by the CCL conditions. Using the general pressure solution (4.2.11) in the CCL pressure and flow rate equations:

Pressure CCL:

$$
A_{j-1}^{1} K_{0}\left(\sigma_{(j-1) 1} r_{w}\right)+B_{j-1}^{1} I_{0}\left(\sigma_{(j-1) 1} r_{w}\right)=A_{j}^{1} K_{0}\left(\sigma_{j 1} r_{w}\right)+B_{j}^{1} I_{0}\left(\sigma_{j 1} r_{w}\right)
$$

for $j=2, \ldots, n$.

Flow rate CCL:

$$
\sum_{j=1}^{n} \sigma_{j 1} \kappa_{j 1}\left(-A_{j}^{1} K_{1}\left(\sigma_{j 1} r_{w}\right)+B_{j}^{1} I_{1}\left(\sigma_{j 1} r_{w}\right)\right)=-\frac{q \mu}{2 \pi r_{w} u}
$$

The other equations left are given by the CCR equations. Using pressure solutions (4.2.11) and (4.2.12) in the CCR pressure and flow rate equations:

Pressure CCR:

$A_{j}^{i-1} K_{0}\left(\sigma_{j(i-1)} r_{j(i-1)}\right)+B_{j}^{i-1} I_{0}\left(\sigma_{j(i-1)} r_{j(i-1)}\right)=A_{j}^{i} K_{0}\left(\sigma_{j i} r_{j(i-1)}\right)+B_{j}^{i} I_{0}\left(\sigma_{j i} r_{j(i-1)}\right)$

for $i=2, \ldots, m-1$.

$$
A_{j}^{i-1} K_{0}\left(\sigma_{j(i-1)} r_{j(i-1)}\right)+B_{j}^{i-1} I_{0}\left(\sigma_{j(i-1)} r_{j(i-1)}\right)=A_{j}^{i} K_{0}\left(\sigma_{j i} r_{j(i-1)}\right)
$$

for $i=m$.

Flow rate CCR:

$A_{j}^{i-1} K_{1}\left(\sigma_{j(i-1)} r_{j(i-1)}\right)-B_{j}^{i-1} I_{1}\left(\sigma_{j(i-1)} r_{j(i-1)}\right)=s\left(A_{j}^{i} K_{1}\left(\sigma_{j i} r_{j(i-1)}\right)-B_{j}^{i} I_{1}\left(\sigma_{j i} r_{j(i-1)}\right)\right)$

for $i=2, \ldots, m-1$.

$$
A_{j}^{i-1} K_{1}\left(\sigma_{j(i-1)} r_{j(i-1)}\right)-B_{j}^{i-1} I_{1}\left(\sigma_{j(i-1)} r_{j(i-1)}\right)=s\left(\left(A_{j}^{i} K_{1}\left(\sigma_{j i} r_{j(i-1)}\right)\right)\right.
$$

for $i=m$. 
Chapter 4. Pressure Response Considering Formation Crossflow in Multilayered

With $s=\frac{\kappa_{j i} \sigma_{j i}}{\kappa_{j(i-1)} \sigma_{j(i-1)}}$ in (4.2.21) and (4.2.22). Now, using relations (4.2.17) through (4.2.22), a linear system can be set up in order to calculate the pressure coefficients:

$$
\left[\begin{array}{c}
A_{1}^{1} \\
B_{1}^{1} \\
A_{1}^{2} \\
B_{1}^{2} \\
\vdots \\
A_{1}^{m_{1}} \\
A_{2}^{1} \\
B_{2}^{1} \\
A_{2}^{2} \\
B_{2}^{2} \\
\vdots \\
A_{2}^{m_{2}} \\
\vdots \\
A_{n}^{1} \\
B_{n}^{1} \\
A_{n}^{2} \\
B_{n}^{2} \\
\vdots \\
A_{n}^{m_{n}}
\end{array}\right]=M^{-1}\left[\begin{array}{c}
-q \mu /\left(2 \pi r_{w} u\right) \\
0 \\
0 \\
0 \\
\vdots \\
0 \\
0 \\
0 \\
0 \\
0 \\
\vdots \\
0 \\
\vdots \\
0 \\
0 \\
0 \\
0 \\
\vdots \\
0
\end{array}\right]
$$

Where the matrix $M$ above is defined by the $(2 m-1) n$ equations $(4.2 .17)$ to (4.2.22). And using coefficients $A_{1}^{1}$ and $B_{1}^{1}$ is possible to find a solution for the pressure variation at the wellbore in the Laplace field. 


\section{5 \\ Pressure Response Considering Formation Crossflow in Mul- tilayered Reservoirs Under Two Phase Flow}

Before the injectivity test begins, the reservoir is filled with single phase liquid (oil), then, the water injection in the oil reservoir begins and the two phase fluid flow scenario is established. The single phase flow was analyzed in the previous chapters. Now, it is assumed that there are both water and oil phases in the reservoir, that is, a two phase flow. This work is considering a piston-like flow, i.e., the water front in each layer advances uniformly.

A single permeability per layer is considered. However, even though the rock properties within each layer are the same, there are different composition properties within them now, such as viscosity and the permeability related to each fluid. Hence, this two phase flow model can be represented by a two non fixed regions model, with the water region increasing with time.

Because of their similarities, the single phase model for composite reservoirs will be used in order to model the two phase case. However, for this case consider $\Delta p_{j i}=p_{j i}-p_{i}$.

Notice that for this case, there is only one semipermeability coefficient $\left(X_{j}\right)$ for each adjacency of layers. That statement is true even though this model is treated as a two regions model, because $X_{j}$ depends only on the rock properties of layers $j$ and $j+1$ and of the shale that connects them, which for this case, do not change.

\section{1}

\section{Two Layered Model}

It will be assumed that the reservoir is composed by two layers, with both water and oil in them and these fluids represent 2 distinct regions in each layer. Constant flow rates and thicknesses will be considered and there is formation crossflow within the layers. In each layer, region 1 will represent the region that contains the water being injected and region 2 the region that contains oil only. Figure 5.1 represents that system for a layer: 


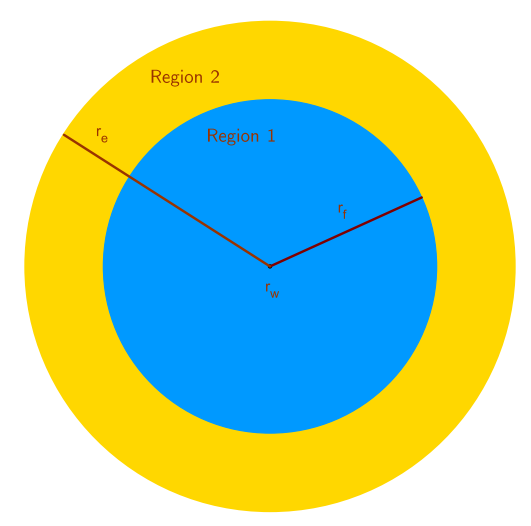

Figure 5.1: Two phase fluid flow in a reservoir

First, consider the volume injected in layer $j$ :

$$
v_{j}(t)=\int_{0}^{t} q_{j}\left(t^{\prime}\right) d t^{\prime}
$$

To calculate the water front radius $\left(r_{F}\right)$ for each layer $j$ the Buckley-Leverett formula for cylindrical geometry is considered:

$$
r_{j F}(t)=\sqrt{\left(r_{w}\right)^{2}+\frac{v_{j} B_{w}}{24 \pi \phi_{j} h_{j}} f_{w j}^{\prime} t}
$$

Where $f_{w j}^{\prime}$ represents the derivative of the curve of fractional flow of water at layer $j$ at each point of fluid saturation. Since a piston-like flow is considered $f_{w j}^{\prime}$ is given by:

$$
f_{w j}^{\prime}=\frac{1}{1-S_{o r}^{j}-S_{w i}^{j}}
$$

Here $S_{F}^{j}$ is the fluid saturation for given time and radius in layer $j$. The water and oil fluid mobility of layer $j\left(\hat{\lambda}_{j}^{F}\right)$, used in the IBC, are defined as:

$$
\hat{\lambda}_{j}^{F}=\frac{k_{j}^{r F} k_{j}}{\mu^{F}}, \text { where } F=w, o
$$


Here $k_{j}^{r F}$ is the relative permeability of layer $j$ to phase $F$ and $k_{j}$ is layer $j$ permeability.

Notice that the water front radius can be different in each layer:

$$
\sqrt{\left(r_{w}\right)^{2}+\frac{v_{1} B_{w}}{24 \pi \phi_{1} h_{1}} f_{w 1}^{\prime}} t \neq \sqrt{\left(r_{w}\right)^{2}+\frac{v_{2} B_{w}}{24 \pi \phi_{2} h_{2}} f_{w 2}^{\prime} t}
$$
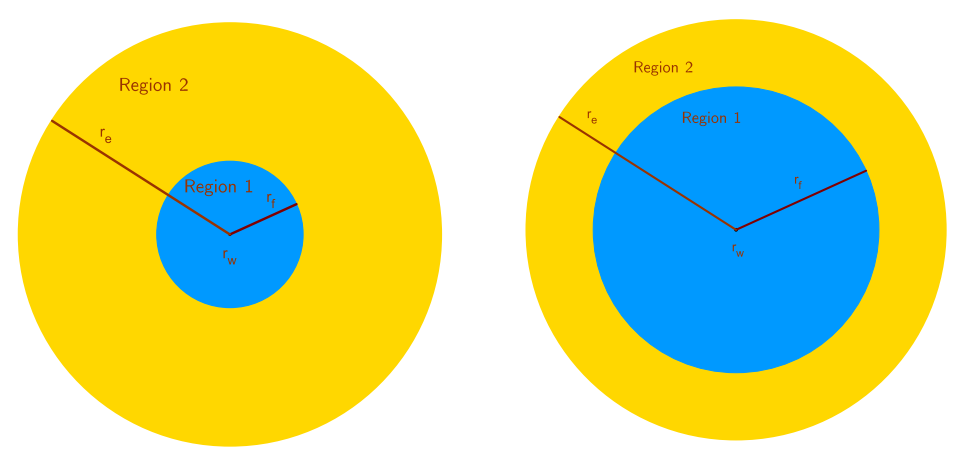

Figure 5.2: Water injection behavior at layer 1 (left) and layer 2 (right)

If one considers the previous equation (5.1.5) to be true, does not imply that a third artificial region will be considered like in the previous chapters 3 and 4 . That is because, the definition of the semipermeability coefficient considered in this work, only considers the rock properties. Since for this case a non composite reservoir is considered, there is a single semipermeability coefficient.

The coefficient of semipermeability $X_{1}$ that relates layers 1 and 2 is defined as in [9]:

$$
X_{1}=\frac{2}{2\left[\left(\Delta h_{1}\right) / k_{v 1}\right]+\chi_{2}+\chi_{1}}
$$

Where each $\chi_{j}$ is defined as:

$$
\chi_{j}=\frac{h_{j}}{k_{z j}}
$$

Where $k_{z j}$ is the absolute vertical permeability for layer $j=1,2$.

The following systems of equations model this problem considering two layers: 


\section{Layer 1:}

Region 1:

$P D E: \kappa_{1} \nabla^{2} \Delta p_{11}=\omega_{1} c_{t} \mu^{w} \frac{\partial \Delta p_{11}(r, t)}{\partial t}-\left(\Delta p_{21}-\Delta p_{11}\right) X_{1} ; r_{w}<r<r_{1 F}, t>0$

IC $: \Delta p_{11}(r, 0)=0$

$I B C: q_{11}=-\left.2 \pi \hat{\lambda}_{w}^{1} h\left(r \frac{\partial \Delta p_{11}}{\partial r}\right)\right|_{r=r_{w}}$

Region 2:

$$
\begin{aligned}
& P D E: \kappa_{1} \nabla^{2} \Delta p_{12}=\omega_{1} c_{t} \mu^{o} \frac{\partial \Delta p_{12}(r, t)}{\partial t}-\left(\Delta p_{22}-\Delta p_{12}\right) X_{1} ; r>r_{1 F}, t>0 \\
& I C: \Delta p_{12}(r, 0)=0 \\
& E B C: \lim _{r \rightarrow \infty} \Delta p_{12}(r=\infty, t)=0
\end{aligned}
$$

The coupling conditions between regions 1 and 2 (CCR) are given by the flow rate and pressure equalities at the water front radius $\left(r_{1 F}\right)$ :

$$
\mathrm{CCR}:\left\{\begin{array}{l}
\Delta p_{11}\left(r_{1 F}, t\right)=\Delta p_{12}\left(r_{1 F}, t\right) \\
\left.\left(r \frac{\partial \Delta p_{11}}{\partial r}\right)\right|_{r=r_{1 F}}=\left.\frac{\hat{\lambda}_{o}^{1}}{\hat{\lambda}_{w}^{1}}\left(r \frac{\partial \Delta p_{12}}{\partial r}\right)\right|_{r=r_{1 F}}
\end{array}\right.
$$

\section{Layer 2:}

Region 1:

$P D E: \kappa_{2} \nabla^{2} \Delta p_{21}=\omega_{2} c_{t} \mu^{w} \frac{\partial \Delta p_{21}(r, t)}{\partial t}-\left(\Delta p_{21}-\Delta p_{11}\right) X_{1} ; r_{w}<r<r_{2 F}, t>0$

IC $: \Delta p_{21}(r, 0)=0$

$I B C: q_{21}=-\left.2 \pi \hat{\lambda}_{w}^{2} h\left(r \frac{\partial \Delta p_{21}}{\partial r}\right)\right|_{r=r_{w}}$ 
Region 2:

$$
\begin{aligned}
& P D E: \kappa_{2} \nabla^{2} \Delta p_{22}=\omega_{2} c_{t} \mu^{o} \frac{\partial \Delta p_{22}(r, t)}{\partial t}-\left(\Delta p_{22}-\Delta p_{12}\right) X_{1} ; r>r_{2 F}, t>0 \\
& I C: \Delta p_{22}(r, 0)=0 \\
& E B C: \lim _{r \rightarrow \infty} \Delta p_{22}(r=\infty, t)=0
\end{aligned}
$$

As in layer 1, the layer 2 coupling conditions between regions are defined as:

$$
\mathrm{CCR}:\left\{\begin{array}{l}
\Delta p_{21}\left(r=r_{2 F}, t\right)=\Delta p_{22}\left(r=r_{2 F}, t\right) \\
\left.\left(r \frac{\partial p_{21}}{\partial r}\right)\right|_{r=r_{2 F}}=\left.\frac{\hat{\lambda}_{o}^{1}}{\hat{\lambda}_{w}^{2}}\left(r \frac{\partial p_{22}}{\partial r}\right)\right|_{r=r_{2 F}}
\end{array}\right.
$$

Between layers, there is also a coupling condition, the CCL. This condition is obtained considering that the pressure drop in both layers is equal at the well and that the flow rate at the well is given by the sum of the flow rates of each layer, that is:

$$
C C L:\left\{\begin{array}{l}
\Delta p_{11}\left(r=r_{w}, t\right)=\Delta p_{21}\left(r=r_{w}, t\right) \\
q=q_{1}+q_{2}
\end{array}\right.
$$

The flow rate equation can be rewritten using Darcy's law:

$$
\left.\left(r \frac{\partial \Delta p_{j 1}}{\partial r}\right)\right|_{r=r_{w}}=-\frac{q_{j 1}}{2 \pi r_{w}\left(\hat{\lambda}_{w}^{1} h_{1}+\hat{\lambda}_{w}^{2} h_{2}\right)}
$$

In the Laplace field the equations (5.1.8) through (5.1.22) can be rewritten. 
Chapter 5. Pressure Response Considering Formation Crossflow in Multilayered

\section{Layer 1:}

Region 1:

$P D E: \kappa_{1} \nabla^{2} \Delta \bar{p}_{11}=\omega_{1} c_{t} \mu^{w} u \Delta \bar{p}_{11}-\left(\Delta \bar{p}_{21}-\Delta \bar{p}_{11}\right) X_{1} ; r_{w}<r<r_{1 F}, t>0$

IC $: \Delta \bar{p}_{11}(r, 0)=0$

$I B C: \quad q_{11}=-\left.2 \pi \hat{\lambda}_{w}^{1} h\left(r \frac{\partial \Delta \bar{p}_{11}}{\partial r}\right)\right|_{r=r_{w}}$

Region 2:

$$
\begin{aligned}
& P D E: \kappa_{1} \nabla^{2} \Delta \bar{p}_{12}=\omega_{1} c_{t} \mu^{o} u \Delta \bar{p}_{12}-\left(\Delta \bar{p}_{22}-\Delta \bar{p}_{12}\right) X_{1} ; r>r_{1 F}, t>0 \\
& I C: \Delta \bar{p}_{12}(r, 0)=0 \\
& E B C: \lim _{r \rightarrow \infty} \Delta \bar{p}_{12}(r, t)=0
\end{aligned}
$$

\section{Layer 2:}

Region 1:

$P D E: \kappa_{2} \nabla^{2} \Delta \bar{p}_{21}=\omega_{2} c_{t} \mu^{w} u \Delta \bar{p}_{21}-\left(\Delta \bar{p}_{21}-\Delta \bar{p}_{11}\right) X_{1} ; r_{w}<r<r_{2 F}, t>0$

$I C: \Delta \bar{p}_{21}(r, 0)=0$

$I B C: q_{21}=-\left.2 \pi \hat{\lambda}_{w}^{2} h\left(r \frac{\partial \Delta \bar{p}_{21}}{\partial r}\right)\right|_{r=r_{w}}$

Region 2:

$P D E: \kappa_{2} \nabla^{2} \Delta \bar{p}_{22}=\omega_{2} c_{t} \mu^{o} u \Delta \bar{p}_{22}-\left(\Delta \bar{p}_{22}-\Delta \bar{p}_{12}\right) X_{1} ; r>r_{2 F}, t>0$

$I C: \Delta \bar{p}_{22}(r, 0)=0$

$E B C: \lim _{r \rightarrow \infty} \Delta \bar{p}_{22}(r, t)=0$ 


$$
\begin{gathered}
\text { CCR }:\left\{\begin{array}{l}
\Delta \bar{p}_{21}\left(r_{2 F}, t\right)=\Delta \bar{p}_{22}\left(r_{2 F}, t\right) \\
\left.\left(r \frac{\partial \Delta \bar{p}_{21}}{\partial r}\right)\right|_{r=r_{2 F}}=\left.\frac{\hat{\lambda}_{o}^{2}}{\hat{\lambda}_{w}^{2}}\left(r \frac{\partial \Delta \bar{p}_{22}}{\partial r}\right)\right|_{r=r_{2 F}}
\end{array}\right. \\
C C L:\left\{\begin{array}{l}
\Delta \bar{p}_{11}\left(r=r_{w}, t\right)=\Delta \bar{p}_{21}\left(r=r_{w}, t\right) \\
\left.\left(r \frac{\partial \Delta \bar{p}}{\partial r}\right)\right|_{r=r_{w}}=-\frac{q}{2 \pi u r_{w}\left(\hat{\lambda}_{w}^{1} h_{1}+\hat{\lambda}_{w}^{2} h_{2}\right)}
\end{array}\right.
\end{gathered}
$$

In both layers, the general pressure solution, in terms of the Bessel functions, for region 1 is given by:

$$
\Delta \bar{p}_{j 1}=A_{j}^{1} K_{0}\left(r \sigma_{j w}\right)+B_{j}^{1} I_{0}\left(r \sigma_{j w}\right)
$$

And for region 2 is:

$$
\Delta \bar{p}_{j 2}=A_{j}^{2} K_{0}\left(r \sigma_{j o}\right)
$$

For $j=1,2$.

Applying the laplacian in both solutions:

$$
\nabla^{2} \Delta \bar{p}_{j 1}=\sigma_{j w}^{2}\left(A_{j}^{1} K_{0}\left(r \sigma_{j w}\right)+B_{j}^{1} I_{0}\left(r \sigma_{j w}\right)\right)
$$

$$
\nabla^{2} \Delta \bar{p}_{j 2}=\sigma_{j o}^{2}\left(A_{j}^{2} K_{0}\left(r \sigma_{j o}\right)\right)
$$

Replacing the equations above into the PDEs, the following system is encountered, where the non-trivial solution for the pressure vector is wanted: 


$$
\left[\begin{array}{cc}
\kappa_{1} \sigma_{1 F}^{2}-\omega_{1} c_{t} \mu_{1}^{F} u-X_{1} & X_{1} \\
X_{1} & \kappa_{2} \sigma_{2 F}^{2}-\omega_{2} c_{t} \mu_{2}^{F} u-X_{1}
\end{array}\right]\left[\begin{array}{c}
\Delta \bar{p}_{1 F} \\
\Delta \bar{p}_{2 F}
\end{array}\right]=0
$$

For $F=w, o$.

The values for the $\sigma_{j F}$ root values are calculated as the determinant of the matrix in (5.1.43) must vanish. As discussed in the previous chapters, this system can be solved as an algebraic eigenvalue problem.

Now, in order to find the pressure coefficients, the solutions (5.1.39) and (5.1.40) are used into the CCL equations and the CCR equations for Layer 1 and Layer 2:

CCL flow rate equation:

$$
\sum_{j=1}^{2} \sigma_{j w}\left(A_{j}^{1} K_{1}\left(r_{w} \sigma_{j w}\right)-B_{j}^{1} I_{1}\left(r_{w} \sigma_{j w}\right)\right)=-\frac{q}{2 \pi u r_{w}\left(\hat{\lambda}_{w}^{1} h_{1}+\hat{\lambda}_{w}^{2} h_{2}\right)}
$$

CCL pressure equation:

$$
A_{1}^{1} K_{0}\left(r_{w} \sigma_{1 w}\right)+B_{1}^{1} I_{0}\left(r_{w} \sigma_{1 w}\right)=A_{2}^{1} K_{0}\left(r_{w} \sigma_{2 w}\right)+B_{2}^{1} I_{0}\left(r_{w} \sigma_{2 w}\right)
$$

CCR flow rate equation for layers $j=1,2$ :

$$
A_{j}^{1} K_{1}\left(r_{j F} \sigma_{j w}\right)-B_{j}^{1} I_{1}\left(r_{j F} \sigma_{j w}\right)=\frac{\hat{\lambda}_{w}^{j}}{\hat{\lambda}_{w}^{j}} \frac{\sigma_{j o}}{\sigma_{j w}}\left[A_{j}^{2} K_{1}\left(r_{j F} \sigma_{j o}\right)\right]
$$

CCR pressure equation for layers $j=1,2$ :

$$
A_{j}^{1} K_{0}\left(r_{j F} \sigma_{j w}\right)+B_{j}^{1} I_{0}\left(r_{j F} \sigma_{j w}\right)=A_{j}^{2} K_{0}\left(r_{j F} \sigma_{j o}\right)
$$

Using the relations (5.1.44) to (5.1.47) given above, the following linear system to find the coefficients $A_{j}^{1}, B_{j}^{1}$ and $A_{j}^{2}$ is obtained. 
Chapter 5. Pressure Response Considering Formation Crossflow in Multilayered

$M(1: 6,1: 3)=\left[\begin{array}{ccc}\sigma_{1 w} K_{1}\left(r_{w} \sigma_{1 w}\right) & -\sigma_{1 w} I_{1}\left(r_{w} \sigma_{1 w}\right) & 0 \\ K_{0}\left(r_{w} \sigma_{1 w}\right) & I_{0}\left(r_{w} \sigma_{1 w}\right) & 0 \\ K_{1}\left(r_{1 F} \sigma_{1 w}\right) & -I_{1}\left(r_{1 F} \sigma_{1 w}\right) & -\frac{\hat{\lambda}_{o}^{1} \sigma_{1 o}}{\hat{\lambda}_{w}^{1} \sigma_{1 w}} K_{1}\left(r_{1 F} \sigma_{1 o}\right) \\ K_{0}\left(r_{1 F} \sigma_{1 w}\right) & I_{0}\left(r_{1 F} \sigma_{1 w}\right) & -K_{0}\left(r_{1 F} \sigma_{1 o}\right) \\ 0 & 0 & 0 \\ 0 & 0 & 0\end{array}\right]$

$M(1: 6,4: 6)=\left[\begin{array}{ccc}\sigma_{2 w} K_{1}\left(r_{w} \sigma_{2 w}\right) & -\sigma_{2 w} I_{1}\left(r_{w} \sigma_{2 w}\right) & 0 \\ -K_{0}\left(r_{w} \sigma_{2 w}\right) & -I_{0}\left(r_{w} \sigma_{2 w}\right) & 0 \\ 0 & 0 & 0 \\ 0 & 0 & 0 \\ K_{1}\left(r_{2 F} \sigma_{2 w}\right) & -I_{1}\left(r_{2 F} \sigma_{2 w}\right) & -\frac{\hat{\lambda}_{o}^{2} \sigma_{2 o}}{\hat{\lambda}_{w}^{2} \sigma_{2 w}} K_{1}\left(r_{2 F} \sigma_{2 o}\right) \\ K_{0}\left(r_{2 F} \sigma_{2 w}\right) & I_{0}\left(r_{2 F} \sigma_{2 w}\right) & -K_{0}\left(r_{2 F} \sigma_{2 o}\right)\end{array}\right]$

$\left[\begin{array}{c}A_{1}^{1} \\ B_{1}^{1} \\ A_{1}^{2} \\ A_{2}^{1} \\ B_{2}^{1} \\ A_{2}^{2}\end{array}\right]=M^{-1}\left[\begin{array}{c}\frac{q}{2 \pi u r_{w}\left(\hat{\lambda}_{w}^{1} h_{1}+\hat{\lambda}_{w}^{2} h_{2}\right)} \\ 0 \\ 0 \\ 0 \\ 0 \\ 0\end{array}\right]$

And, finally, it is possible to calculate the pressure variation at the wellbore: 


$$
\Delta \bar{p}_{11}=A_{1}^{1} K_{0}\left(\sigma_{1 w} r_{w}\right)+B_{1}^{1} I_{0}\left(\sigma_{1 w} r_{w}\right)
$$

Pressure profile is given by equation (5.1.48) in the Laplace field. Using the Stehfest Algorithm[14], this solution is given in the real field.

\section{2}

\section{Multilayered Model}

The problem considered in section 5.1 is similar to the one for two layers and two regions of equal radii for single phase flow studied in section 3.1. Hence, the two phase flow model considering $n$ layers is analogous to the single phase flow considering $n$ layers and two regions model studied in section 3.2.

The general pressure solution for this case in the Laplace field is given by:

For Region 1 (water) is:

$$
\Delta \bar{p}_{j 1}=A_{j}^{1} K_{0}\left(r_{w} \sigma_{j w}\right)+B_{j}^{1} I_{0}\left(r_{w} \sigma_{j w}\right)
$$

And for Region 2 (oil) is:

$$
\Delta \bar{p}_{j 2}=A_{j}^{2} K_{0}\left(r_{w} \sigma_{j o}\right)
$$

For $j=1,2, \ldots, n$ now.

Replacing pressure solutions (5.2.1) and (5.2.2) in the PDE's for this problem, one can obtain a homogeneous system $n \times n$ for each region of fluid $F$, which is used to calculate the $\sigma_{j F}$ root values:

$$
\left(\kappa_{j} \sigma_{j F}^{2}-\omega_{j} c_{t} \mu_{j}^{F} u-X_{j-1}-X_{j}\right) \Delta \bar{p}_{j F}+X_{j-1} \Delta \bar{p}_{(j-1) F}-X_{j} \Delta \bar{p}_{(j+1) F}=0
$$

For $j=1,2, \ldots, n$ and $F=w, o$. 
After calculating the $\sigma_{j F}$ root values by vanishing the determinant of the matrix of the linear system (5.2.3), it is left to find the pressure coefficients in order to calculate the pressure variation.

The coupling conditions of the layers and the regions are used to find the pressure coefficients. They are given by:

$$
C C L=\left\{\begin{array}{l}
-\frac{q}{2 \pi u\left(\hat{\lambda}_{w}^{1} h_{1}+\hat{\lambda}_{w}^{2} h_{2}+\ldots+\hat{\lambda}_{w}^{n} h_{n}\right)}=\left.\sum_{j=1}^{n} \frac{\partial \Delta \bar{p}_{j 1}}{\partial r}\right|_{r=r_{w}} \\
\Delta \bar{p}_{11}\left(r=r_{w}, t\right)=\Delta \bar{p}_{21}\left(r=r_{w}, t\right) \\
\Delta \bar{p}_{21}\left(r=r_{w}, t\right)=\Delta \bar{p}_{31}\left(r=r_{w}, t\right) \\
\vdots \\
\Delta \bar{p}_{(n-1) 1}\left(r=r_{w}, t\right)=\Delta \bar{p}_{n 1}\left(r=r_{w}, t\right)
\end{array}\right.
$$

$$
C C R=\left\{\begin{array}{l}
\Delta \bar{p}_{11}\left(r=r_{1 F}, t\right)=\Delta \bar{p}_{12}\left(r=r_{1 F}, t\right) \\
\Delta \bar{p}_{21}\left(r=r_{2 F}, t\right)=\Delta \bar{p}_{22}\left(r=r_{2 F}, t\right) \\
\vdots \\
\Delta \bar{p}_{n 1}\left(r=r_{n F}, t\right)=\Delta \bar{p}_{n 2}\left(r=r_{n F}, t\right) \\
\left.\left(r \frac{\partial \Delta \bar{p}_{11}}{\partial r}\right)\right|_{r=r_{1 F}}=\left.\frac{\hat{\lambda}_{o}^{1}}{\hat{\lambda}_{w}^{1}}\left(r \frac{\partial \Delta \bar{p}_{12}}{\partial r}\right)\right|_{r=r_{1 F}} \\
\left.\left(r \frac{\partial \Delta \bar{p}_{21}}{\partial r}\right)\right|_{r=r_{2 F}}=\left.\frac{\hat{\lambda}_{o}^{2}}{\hat{\lambda}_{w}^{2}}\left(r \frac{\partial \Delta \bar{p}_{22}}{\partial r}\right)\right|_{r=r_{2 F}} \\
\vdots \\
\left.\left(r \frac{\partial \Delta \bar{p}_{n 1}}{\partial r}\right)\right|_{r=r_{n F}}=\left.\frac{\hat{\lambda}_{o}^{n}}{\hat{\lambda}_{w}^{n}}\left(r \frac{\partial \Delta \bar{p}_{n 2}}{\partial r}\right)\right|_{r=r_{n F}}
\end{array}\right.
$$

Replacing equations (5.2.1) and (5.2.2) in the CCL's (5.2.4) and the CCR's (5.2.5), provide a linear system that allows one to find the pressure coefficients and, consequently, the pressure response for the multilayered and two phase flow case. 


\section{6 \\ Results and Discussion}

In this chapter, the accuracy of the solutions proposed in chapters 3 through 5 , will be evaluated. A set of cases regarding those problems were developed in the finite-difference based flow simulator IMEX and then, compared to its respective analytical implemented results.

As an input for the numerical simulation, a radial grid was considered. The grid is more refined in the region closest to the wellbore, which is the region most affected by its presence. Minimum time step was set at $10^{-7} \mathrm{~h}$. The oil model used in the simulator was black oil.

Different cases will be analyzed for each phase fluid flow model, that is, one and two phase models. For all cases, formation crossflow is considered between the adjacent layers.

In order to consider the presence of formation crossflow in the numerical simulator the trans $K$ variable is considered to be 1 . That indicates that the shale between the layers allows the transition of fluid.

For all cases, the following parameters were considered:

- 4 days (96 hours) injection/production period.

- Flow rate at the wellbore was defined as $500 \mathrm{~m}^{3} /$ day $\left(5.79 \times 10^{3} \mathrm{~m}^{3} / \mathrm{s}\right)$.

- Initial pressure was defined as $300 \mathrm{kgf} / \mathrm{cm}^{2}$.

- The wellbore radius was considered to be $r_{w}=0.108 \mathrm{~m}$ in all cases.

- The vertical permeability was considered to be equal to the horizontal permeability for all cases.

- Porosity was defined as $\phi=0.2$ for all cases

The other parameters can be found in tables 6.1 to 6.2 for all cases.

In addition, in the graphs of all cases, besides the pressure change curves for the analytical and numerical solution, the curves of pressure derivatives with respect to the logarithm of time is also present. It is important to analyze the behavior of the pressure derivative as well, in order to properly interpret the results of the test.

Consider the curve composed by blue circles to be the analytical solution for pressure variation and the curve composed by red circles to be the analytical solution for pressure derivative. For the numerical solutions, yellow stars curve represent the pressure variations and purple stars the derivative curve. 


\section{1}

\section{Cases Under Single Phase Flow}

In this section, the reservoir is considered to be filled with a single fluid, oil. For the single phase fluid flow cases, the following parameters are described in table 6.1:

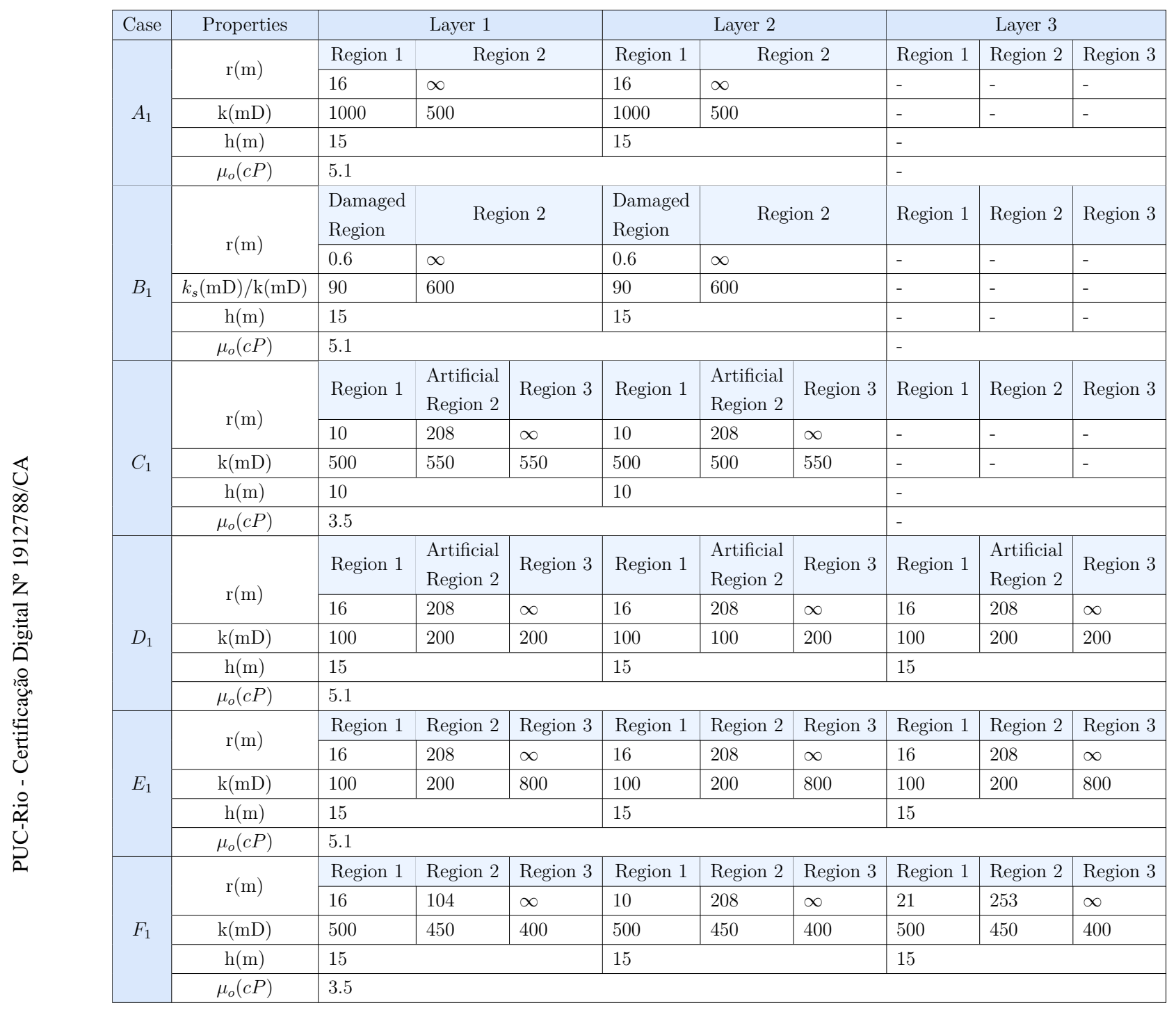

Table 6.1: Analyzed single-phase flow cases

First, case $A_{1}$ is considered. Two layers and two regions are considered for this case, the radii of the regions are considered to be equal. Pressure and pressure derivative curves for the analytical model solution and numerical solution are presented in figure 6.1 for case $A_{1}$ : 


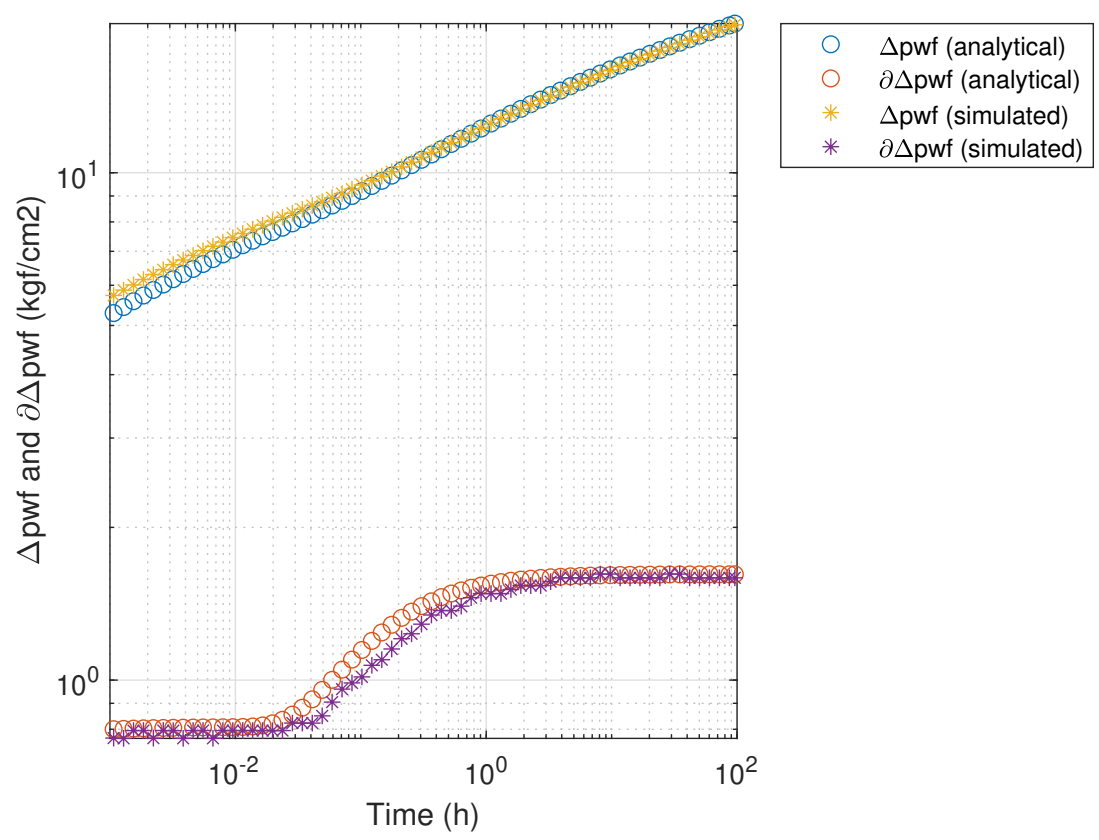

Figure 6.1: Analytical and numerical pressure variation and derivative solutions for case $A_{1}$

These graphs represents the two distinct regions of permeability impacting the pressure and derivative curves.

Region 1 has a permeability value of $1000 \mathrm{mD}$ and the second region has a permeability value of half of that, $500 \mathrm{mD}$. This directly affects the pressure change and its derivative curves. Indeed, for the initial times the pressure curve only notices the presence of the first region of permeability. After that, it reflects the second region, doubling the value of the derivative curve.

It is possible to see a close agreement between the analytical and numerical simulated curves, especially after some time.

Now, case $B_{1}$ is considered. A damaged region near the wellbore is considered. The radius of that region is small and the permeability of the damaged region is considered to be more than 6 times less than the reservoir's permeability. As seen in section 3.5, that indicates that $S>0$, that is, there is damage near the wellbore.

Case $B_{1}$ graphs are given in figure 6.2 : 


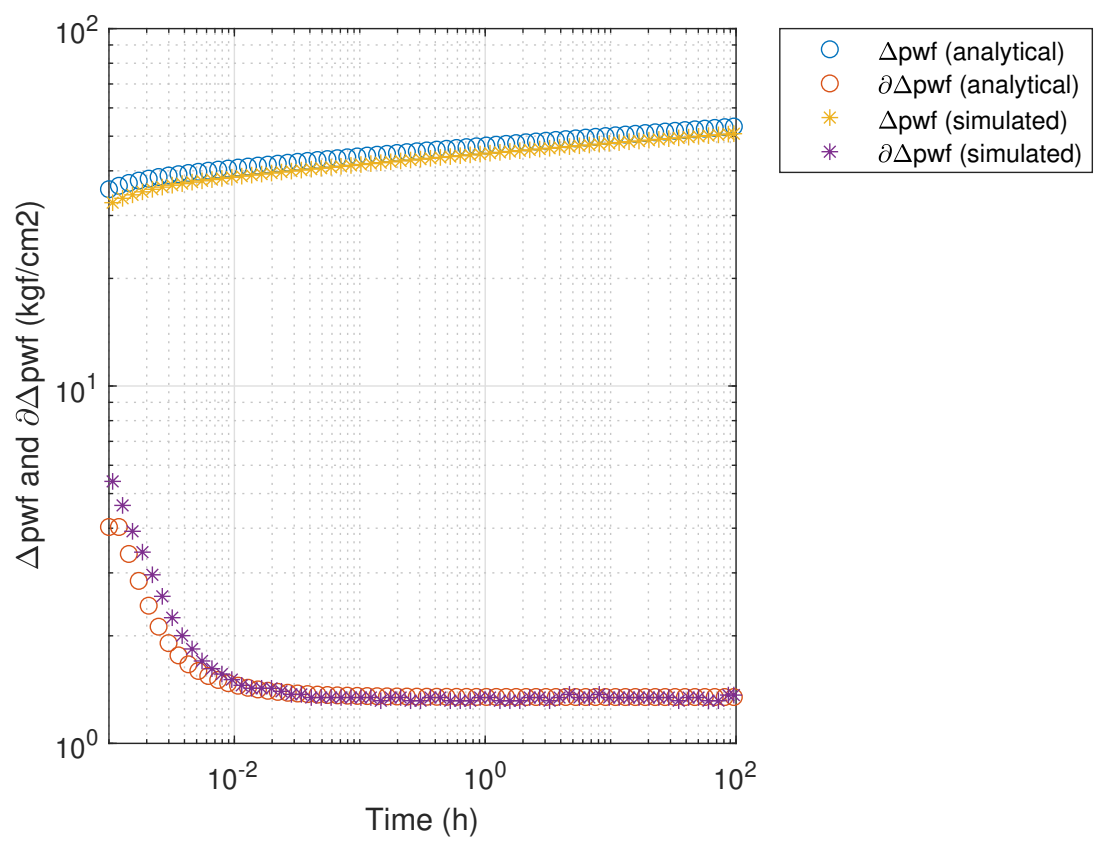

Figure 6.2: Analytical and numerical pressure variation and derivative solutions for case $B_{1}$

The value of the skin $S$ calculated for this case was 9.12, which indicates a highly damaged zone near the wellbore. In the graphs represented in figure 6.2 , it is possible to see the influence of that damaged region on the curves, specially on the derivative curves, which during early times reflects that, apparently, the reservoir only notices the presence of a permeability value equal to $90 \mathrm{mD}$. Then, with time, the curve decreases and stays constant.

There is a small divergence between the analytical model result and the numerical simulator result during early times for the derivative curves and during all times for the pressure curves. However, the behavior of the curves are very similar.

The case $C_{1}$ considers two layers and two regions like in the two previous cases, however, for this case the radii of the regions are distinct. As seen in section 3.3, this problem can be treated as a three regions of equal radii case.

Case $C_{1}$ graphs are given in figure 6.3: 


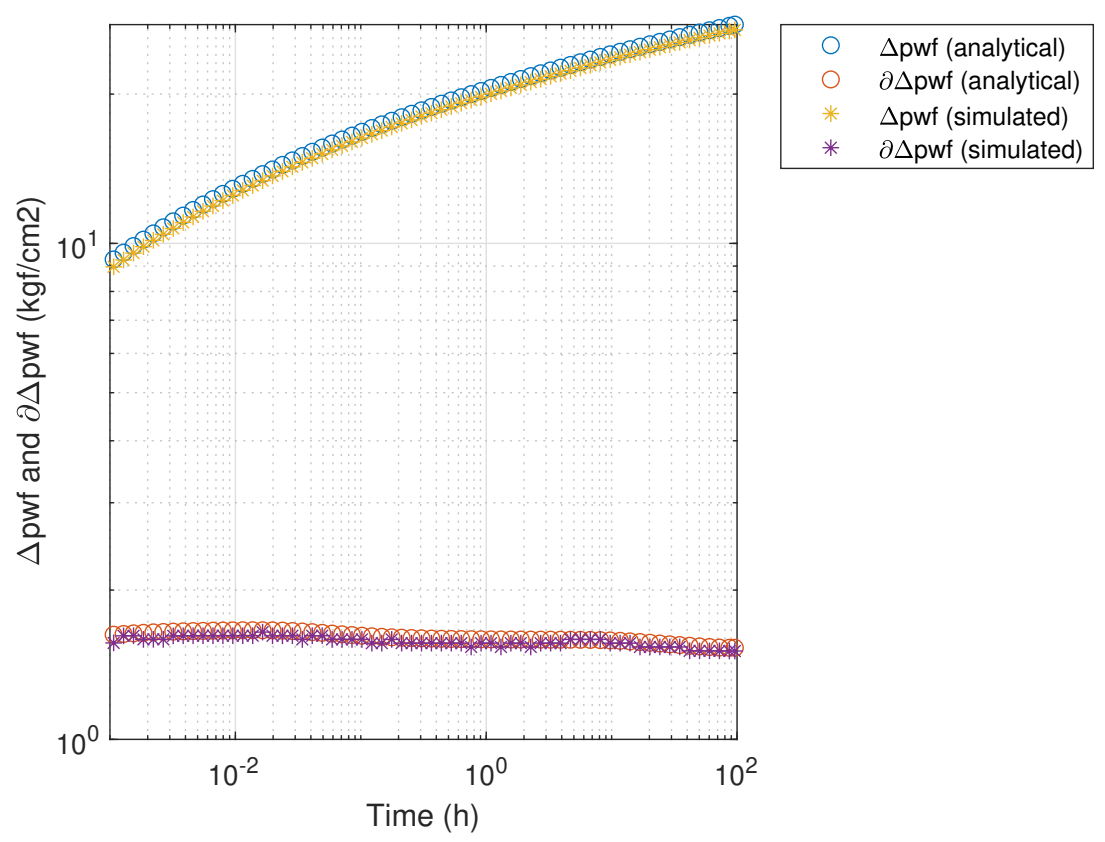

Figure 6.3: Analytical and numerical pressure variation and derivative solutions for case $C_{1}$

The region of permeability $500 \mathrm{mD}$ for layer 1 has $10 \mathrm{~m}$, and in layer 2 , it has $228 \mathrm{~m}$ and the region of permeability $550 \mathrm{mD}$ for layer 1 has $228 \mathrm{~m}$, and in layer 2 , it has $10 \mathrm{~m}$. Hence, the artificial region 2 created has a permeability value of $550 \mathrm{mD}$ for layer 1 and $500 \mathrm{mD}$ for layer 2 and for that region the presence of formation crossflow will have greater impact than in the other regions which have same values of permeability for both layers.

It is possible to see a close agreement between the analytical and the numerical curves.

For case $D_{1}$, three layers are considered and there are two regions of permeability in each one of them, with different radii, hence this case is treated as a three regions with equal radii where region 2 was artificially created.

Case $D_{1}$ graphs are presented in figure 6.4: 


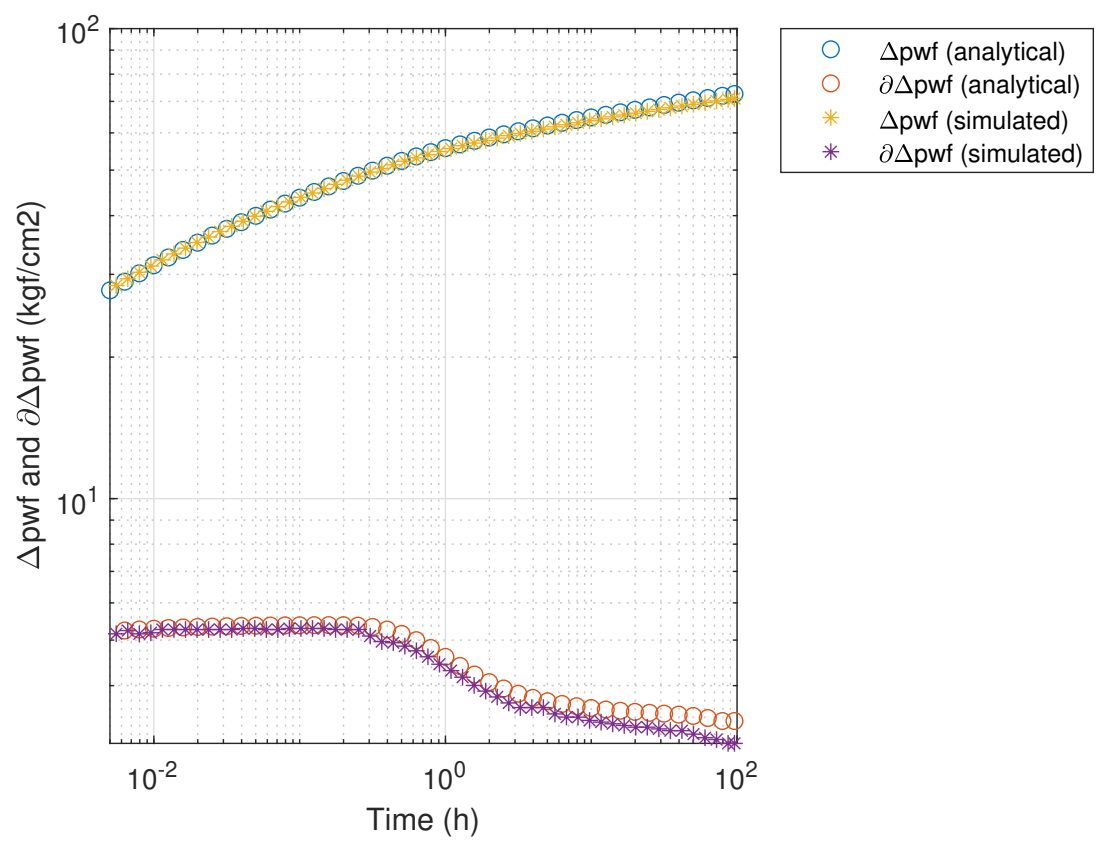

Figure 6.4: Analytical and numerical pressure variation and derivative solutions for case $D_{1}$

This case is similar to case $C_{1}$, in artificially created region 2 for layers 1 and 3 the permeability is equal to $200 \mathrm{mD}$, and in layer 2 it is equal to $100 \mathrm{mD}$. Like in the previous case, the fluid flow in this region causes a decline in the pressure curve and its derivatives.

The presence of formation crossflow in the reservoir causes a decline on the pressure variation curves and its derivatives, because there is more fluid flux, decreasing, that way, the pressure variation in the reservoir.

It is possible to see that, in this case, the numerical simulated curves reflects a greater presence of the formation crossflow, for its curves are more decreased than in the analytical model's. However, the behavior of the curves are still very similar.

It is also possible to see, that the derivative curves reflect the different regions of permeability present in this case.

Case $E_{1}$ is considered now. This case, like in case $D_{1}$, have three layers, however, this case have truly 3 different regions of permeability. Notice that, in our model, case $D_{1}$ (two regions with distinct radii) and case $E_{1}$ (three regions with equal radii) are solved with the same approach.

The graphs for case $E_{1}$ are presented in figure 6.5: 


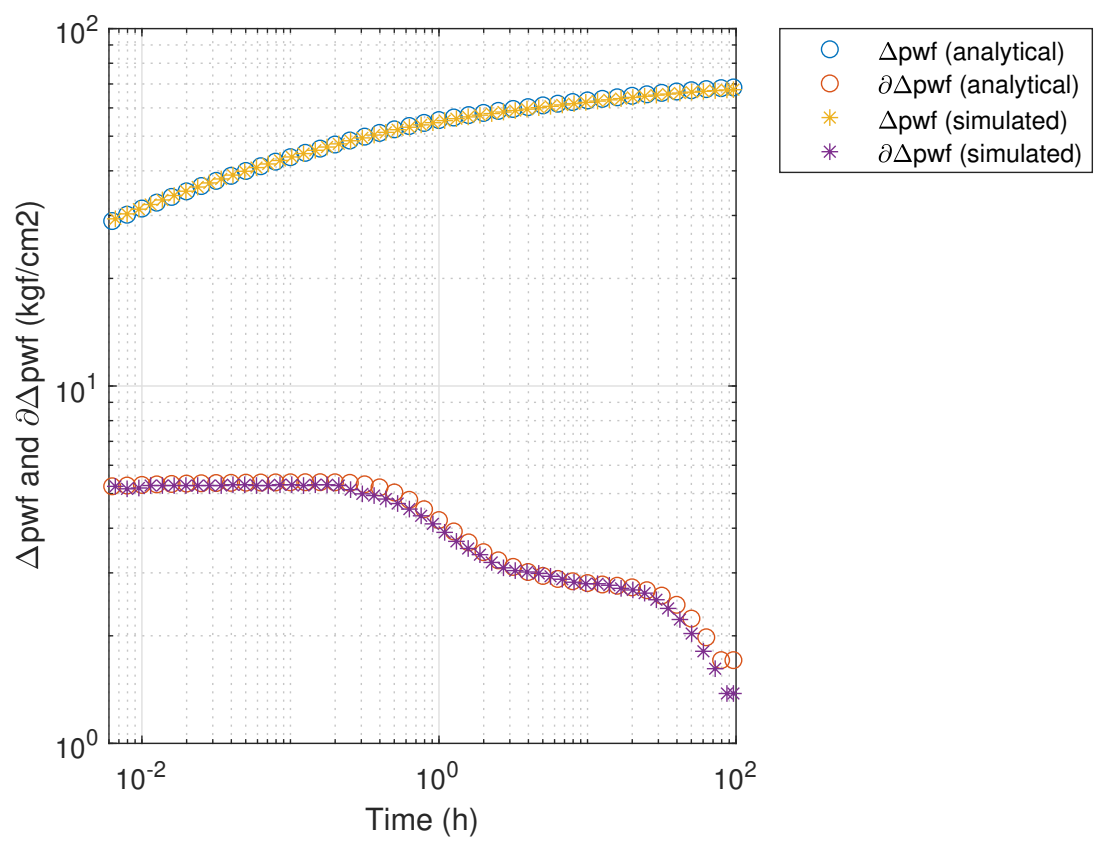

Figure 6.5: Analytical and numerical pressure variation and derivative solutions for case $E_{1}$

It is possible to see that the derivative curves reflect well the three regions. Since all layers have equal properties and there is the presence of formation crossflow, the reservoir behaves like a single layered one [9].

There was a close agreement between the pressure curves and the derivative curves. The region 3 with permeability value of $800 \mathrm{mD}$ causes a significantly decline in the derivative curves. This third region is represented in a constant baseline for later times, not represented in figure 6.5.

Finally, case $F_{1}$ is considered. The model used for that case was presented in chapter 4 . Three layers and three regions with distinct radii are considered. Since the radii for all regions are different, the number of different semipermeability coefficients is 5 for this example.

Case $F_{1}$ graphs are given in figure 6.6: 


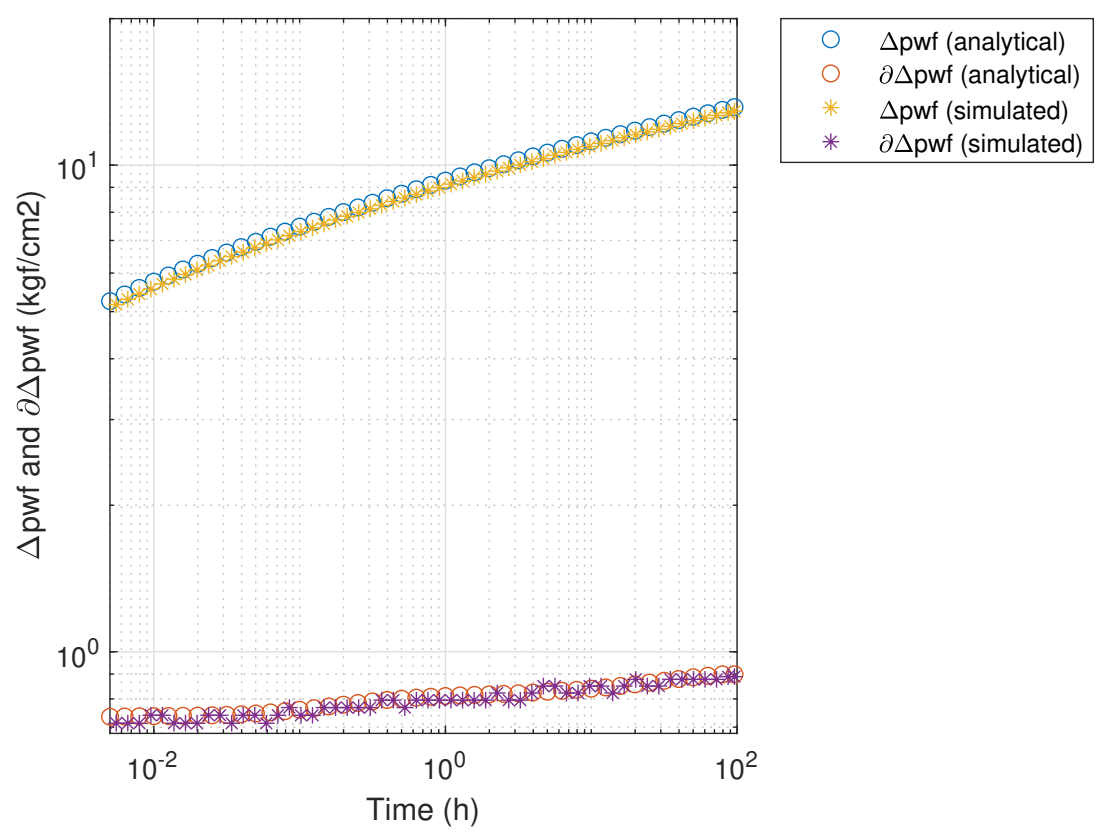

Figure 6.6: Analytical and numerical pressure variation and derivative solutions for case $F_{1}$

It is possible to see a close agreement between the numerical and analytical curves. The fact that the value of permeability at the final regions for all layers is less than the value at the initial regions causes the derivative curves to increase with time.

\section{2}

\section{Cases Under Two Phase Flow}

In this section, the two phase fluid flow cases are considered. The following hypothesis are considered:

- A piston-like flow

- Injection flow rate was defined as $500 \mathrm{~m}^{3} /$ day $\left(5.79 \times 10^{3} \mathrm{~m}^{3} / \mathrm{s}\right)$.

- Water viscosity will always be considered to be $\mu_{w}=0.5$

- In order to best evaluate the efficiency of the model, the mobility ratio is considered as a parameter for all cases in each layer, and it is given by: $\hat{M}_{j}=\frac{\hat{\lambda}_{j}^{w}}{\hat{\lambda}_{j}^{o}}$

- $f_{w j}^{\prime}$ is going to be considered 1 for all layers 
The mobility ratio, is directly connected to the sweep efficiency of a reservoir. Low mobility ratios $(M<1)$ imply in better sweep efficiency, as the interface between phases become more uniform. On the other hand, high mobility ratios lead to uneven fronts, reducing the swept reservoir area[12].

The missing parameters are presented in table 6.2 for the two phase flow cases considered:

\begin{tabular}{|c|c|c|c|c|c|c|}
\hline Case & Properties & Layer 1 & Layer 2 & Layer 3 & Layer 4 & Layer 5 \\
\hline \multirow{4}{*}{$A_{2}$} & $\mathrm{k}(\mathrm{mD})$ & 100 & 100 & - & - & - \\
\hline & $\mathrm{h}(\mathrm{m})$ & 15 & 15 & - & - & - \\
\hline & $\mu_{o}(c P)$ & \multicolumn{2}{|l|}{5.0} & - & - & - \\
\hline & $\hat{M}$ & 10.2 & 10.2 & - & - & - \\
\hline \multirow{4}{*}{$B_{2}$} & $\mathrm{k}(\mathrm{mD})$ & 500 & 1000 & - & - & - \\
\hline & $\mathrm{h}(\mathrm{m})$ & 15 & 15 & - & - & - \\
\hline & $\mu_{o}(\mathrm{cP})$ & \multicolumn{3}{|l|}{5.0} & - & - \\
\hline & $\hat{M}$ & 10.2 & 10.2 & - & - & - \\
\hline \multirow{4}{*}{$C_{2}$} & $\mathrm{k}(\mathrm{mD})$ & 500 & 500 & 500 & - & - \\
\hline & $\mathrm{h}(\mathrm{m})$ & 15 & 15 & 15 & - & - \\
\hline & $\mu_{o}(\mathrm{cP})$ & \multicolumn{3}{|l|}{0.3} & - & - \\
\hline & $\hat{M}$ & 0.6 & 0.6 & 0.6 & - & - \\
\hline \multirow{4}{*}{$D_{2}$} & $\mathrm{k}(\mathrm{mD})$ & 500 & 600 & 700 & - & - \\
\hline & $\mathrm{h}(\mathrm{m})$ & 15 & 15 & 15 & - & - \\
\hline & $\mu_{o}(\mathrm{cP})$ & \multicolumn{3}{|l|}{0.1} & - & - \\
\hline & $\hat{M}$ & 0.2 & 0.2 & 0.2 & - & - \\
\hline \multirow{4}{*}{$E_{2}$} & $\mathrm{k}(\mathrm{mD})$ & 1000 & 600 & 300 & & \\
\hline & $\mathrm{h}(\mathrm{m})$ & 15 & 15 & 15 & - & - \\
\hline & $\mu_{o}(c P)$ & \multicolumn{3}{|l|}{3.5} & - & - \\
\hline & $\hat{M}$ & 7 & 7 & 7 & - & - \\
\hline \multirow{4}{*}{$F_{2}$} & $\mathrm{k}(\mathrm{mD})$ & 100 & 150 & 200 & 250 & 300 \\
\hline & $\mathrm{h}(\mathrm{m})$ & 15 & 15 & 15 & 15 & 15 \\
\hline & $\mu_{0}(c P)$ & \multicolumn{5}{|l|}{5.1} \\
\hline & $\hat{M}$ & 10.2 & 10.2 & 10.2 & 10.2 & 10.2 \\
\hline
\end{tabular}

Table 6.2: Analyzed two phase fluid flow cases

Case $A_{2}$ considers two layers of equal permeability values of $100 \mathrm{mD}$. Figure 6.7 shows the pressure and pressure derivative curves of the analytical model and the numerical simulator: 


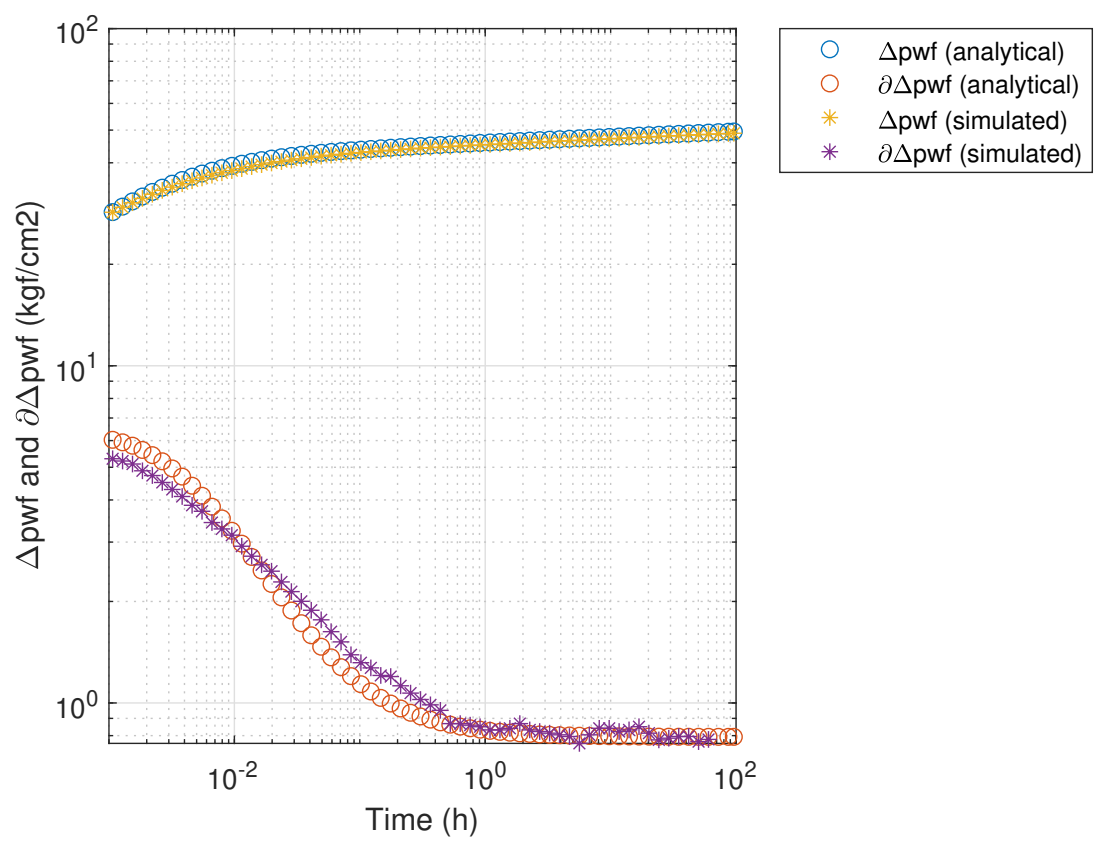

Figure 6.7: Analytical and numerical pressure variation and derivative solutions for case $A_{2}$

It is possible to see a close agreement between these curves, specially the pressure variation curves for the analytical and numerical cases.

Even though there are no different regions of permeability in the layers for this case, the derivative curves behaves as if there were, like in the previous section where different regions of permeabilities were considered. That is because, the regions of equal permeability are filled with different fluids. The first one, water, has a viscosity value of $\mu_{w}=0.5 \mathrm{cP}$, and the second fluid, oil, has viscosity value of $\mu_{o}=5.0 \mathrm{cP}$. These viscosity values causes the value of the mobility ratio $\hat{M}$ to be grater than 1 . Implying on higher pressure derivative values on early times.

Now case $B_{2}$ is analyzed. For this case, a two layer model with different values of permeability in each layer is considered.

The graphs for case $B_{2}$ are given in figure 6.8: 


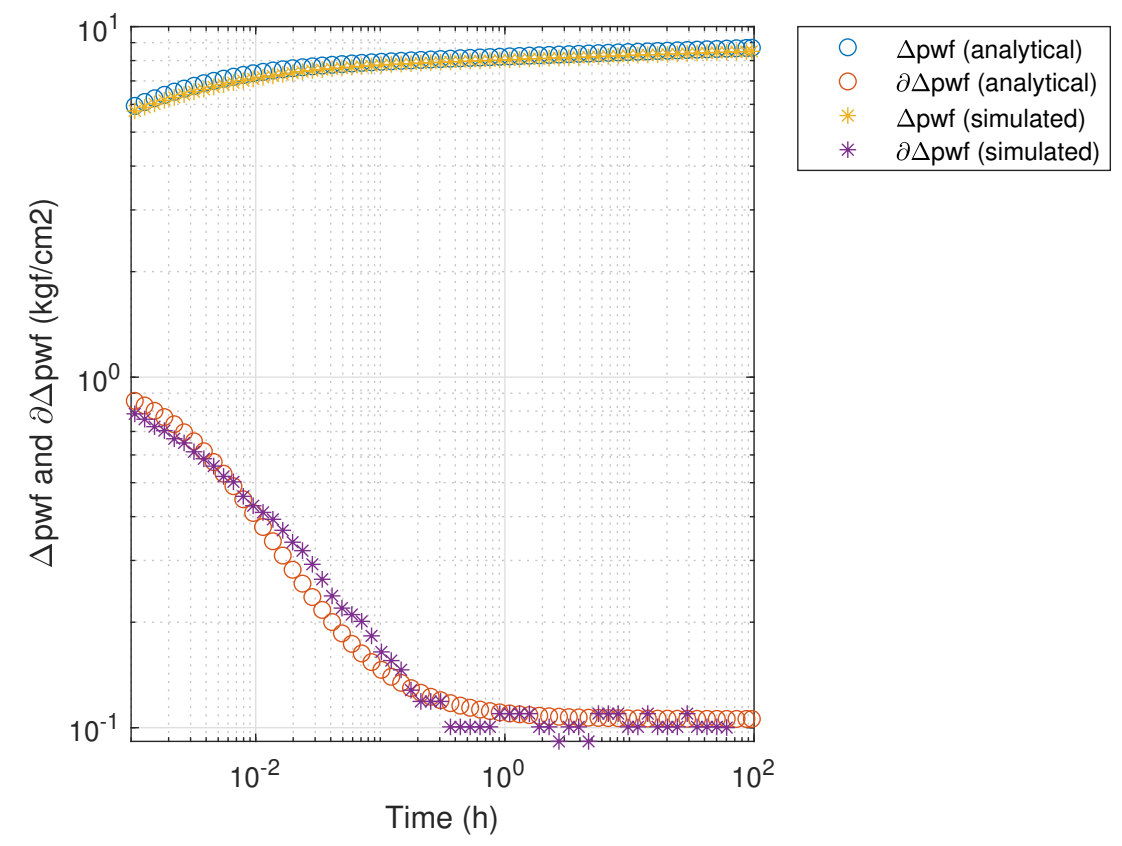

Figure 6.8: Analytical and numerical pressure variation and derivative solutions for case $B_{2}$

It is possible to see a similar behavior to case $A_{2}$, because the values of the mobility ratios $\hat{M}$ are equal. They imply in higher values of pressure derivatives during initial times. It is possible to see that there were a bigger difference in the derivative curves from early times to later times when compared to previous case $A_{2}$, that is because of the different values of permeabilities in layers 1 and 2 . The agreement between the numerical and analytical curves was very close.

Now, case $C_{2}$ is analyzed. A three layer model is considered and the permeability for all layers have the same values. For that case, the viscosity of the oil considered $\left(\mu_{o}=0.3 \mathrm{cP}\right)$ is lower than the water's $\left(\mu_{w}=0.5 \mathrm{cP}\right)$.

Graphs for pressure and pressure derivative for case $C_{2}$ are represented in figure 6.9: 


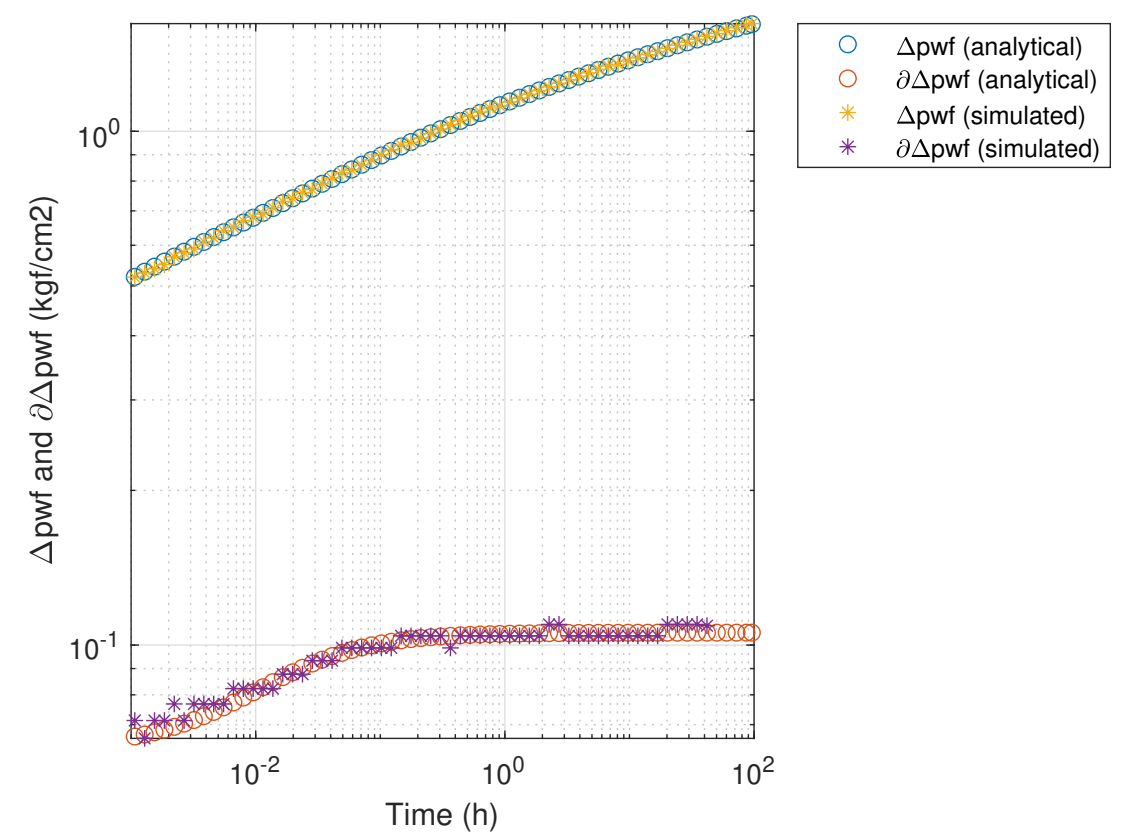

Figure 6.9: Analytical and numerical pressure variation and derivative solutions for case $C_{2}$

The value of $\hat{M}$ for this case is less than 1 (0.6), favorable to the oil flow, improving the sweep efficiency. That directly impacts the pressure derivative curve in a way that the first region, which is filled with water and represented at the end of the graph is higher than the part on the beginning of the curve representing the region filled with oil.

It is also possible to see that the pressure curve is increasing with time. The agreement between analytical and simulated curves were close at all times for this case.

For case $D_{2}$, the oil viscosity considered was $\mu_{o}=0.1 \mathrm{cP}$, which was 5 times smaller than the water's $\left(\mu_{w}=0.5\right)$. Implying on an even smaller value for $\hat{M}$, in this case it was 0.2 . This case also considers different values of permeability per layer.

The graphs for pressure and pressure derivative for case $D_{2}$ are represented in figure 6.10: 


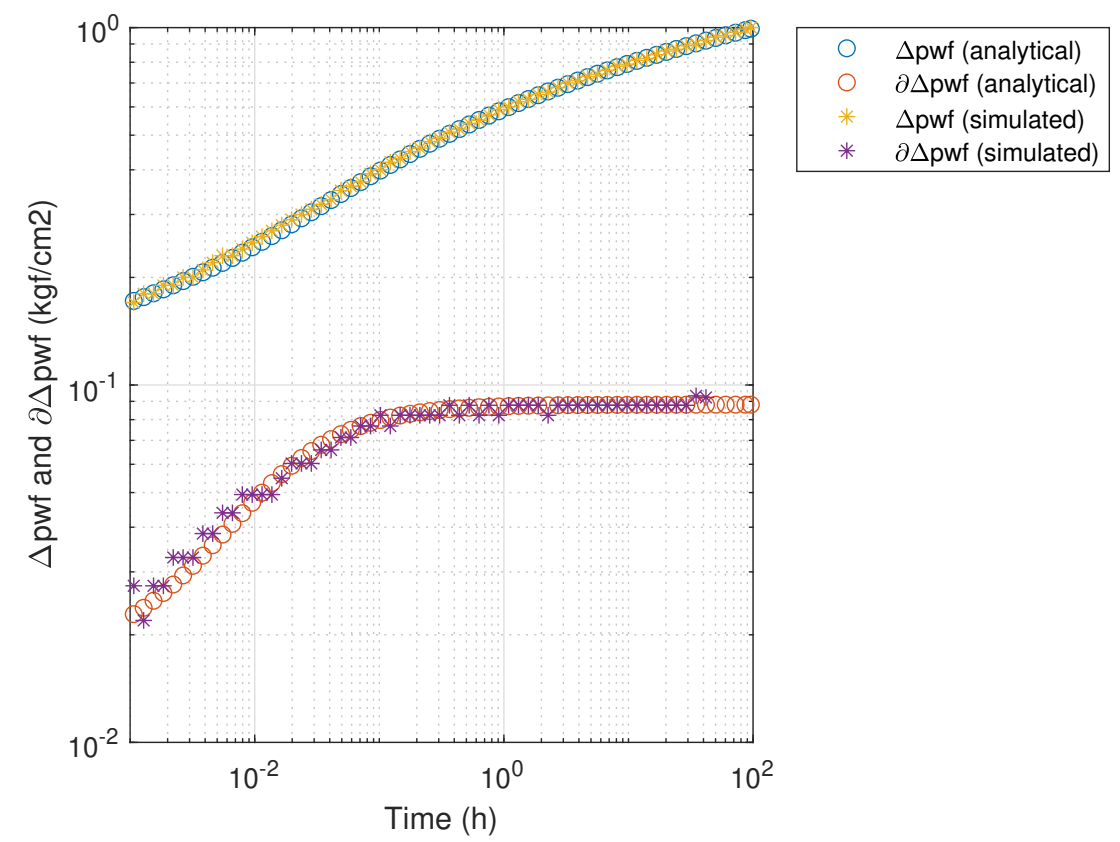

Figure 6.10: Analytical and numerical pressure variation and derivative solutions for case $D_{2}$

It is possible to see that the values in the derivative curves at initial times were five times smaller than in the final times. The pressure curves were increasing, that is because $\hat{M}<<1$ and the sweep efficiency was improved. There was a close agreement between the numerical and analytical curves for this case also.

Now, case $E_{2}$ is analyzed, that case considers three layers with different properties and $\mu_{o}=3.5$.

The graphs for pressure and pressure derivative for this case are presented in figure 6.11: 


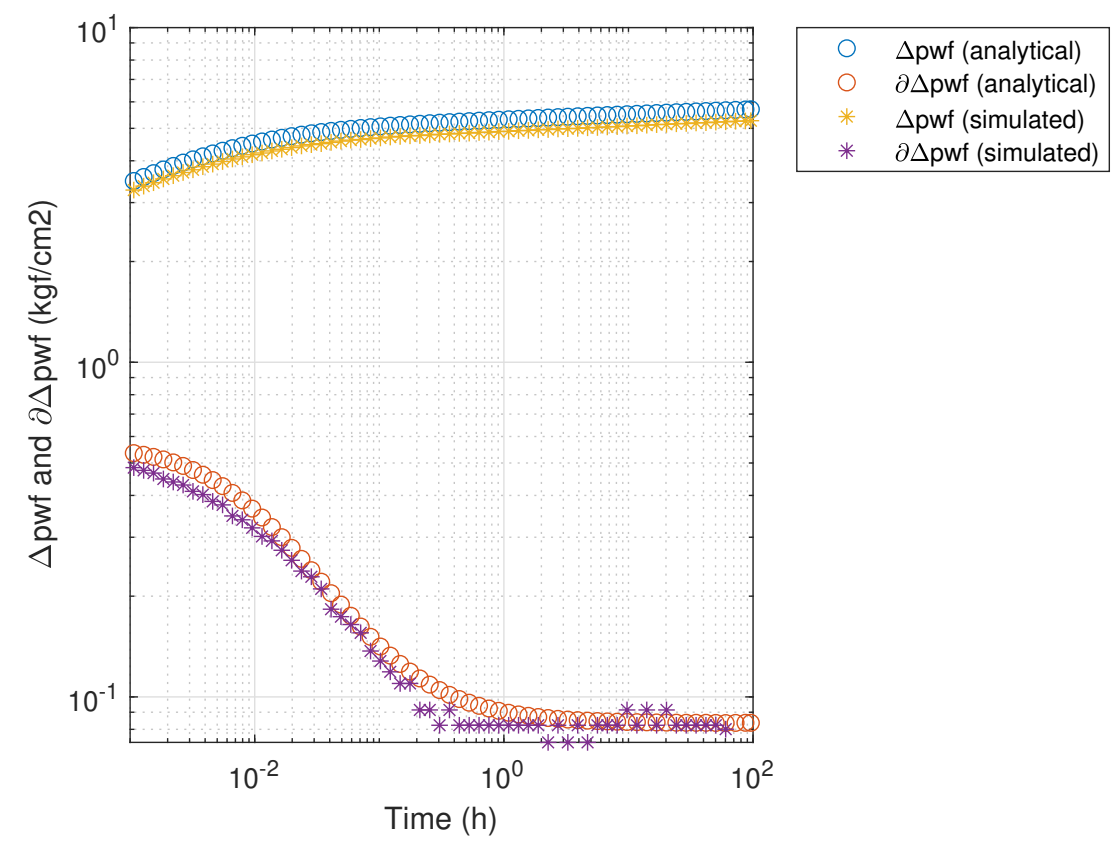

Figure 6.11: Analytical and numerical pressure variation and derivative solutions for case $E_{2}$

Similarly to cases $A_{2}$ and $B_{2}$, for this case $\hat{M}>1$, however, since the difference between the fluids viscosity values is not as big as in cases $A_{2}$ and $B_{2}$, the difference in the values of the derivative curves is not as greater.

There was a small, and apparently constant, difference between the analytical and numerical pressure curves. One reason for that, might be because there is a big difference in the permeabilities values from one layer to another causing a bigger awareness of the presence of formation crossflow in the reservoir, and the way the simulator includes the crossflow is not equal to the way presented in this work. However, the behavior of the curves are still very similar, resulting in a fair approximation.

Finally, case $F_{2}$ considers five layers with different values of permeability and an oil viscosity value of 5.1 .

Figure 6.12 shows the pressure and pressure derivative curves for case $F_{2}$ : 


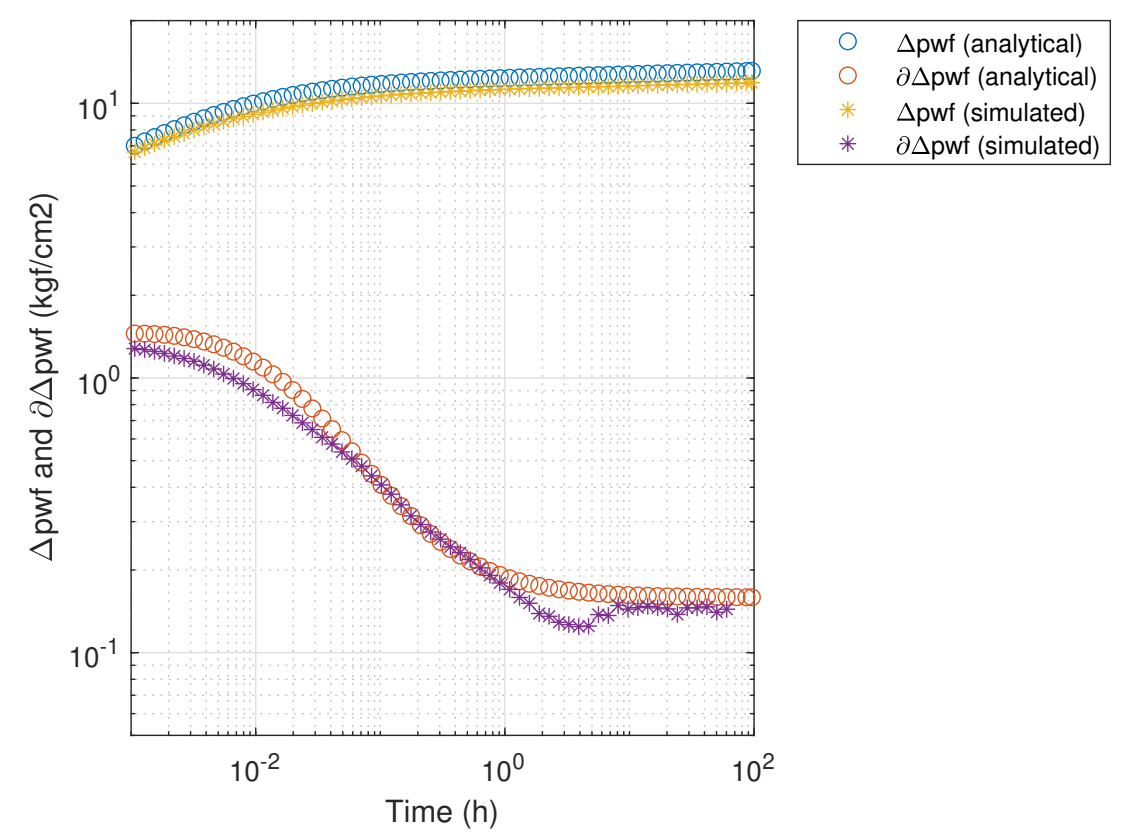

Figure 6.12: Analytical and numerical pressure variation and derivative solutions for case $F_{2}$

The pressure curves show a quite small constant difference for all times and the pressure derivative curve for the numerical simulated case shows a small drop of value during times $1 \mathrm{~h}$ to $4 \mathrm{~h}$. Besides that, the behavior for the analytical and numerical curves are similar and reflects well the regions filled with oil and water also, since $\hat{M}>1$, the flow is favorable to the water resulting on higher pressure derivative values on early times.

\section{3}

\section{Equivalent Permeability}

In a multilayered reservoir, the pressure response is dependent of the equivalent permeability $\left(k_{\text {eq }}\right)$. It is given by:

$$
k_{\mathrm{eq}}=\frac{\sum_{j=1}^{n} k_{j} h_{j}}{\sum_{j=1}^{n} h_{j}}
$$

Since the single phase model presented here reduces the cases of different regions radii into one with regions of equal radii, then the derivative profile enables the computation of equivalent permeabilities that combine the respective regions of the layers. That is, to obtain the first equivalent permeability, region 1 of layer 1 is combined with region 1 of layer 2 and so on. 
Using the source line logarithmic approximation, the following equation is used to estimate the equivalent permeability:

$$
\bar{k}_{\mathrm{eq}}=\frac{q \mu_{o}}{2 h_{T} m}
$$

Where $m$ is the constant derivative level. It can be calculated from the lines in the semilog graph for each analytical case.

For the two phase flow, the following equation is used to estimate the equivalent permeability:

$$
\bar{k}_{\mathrm{eq}}=\frac{q}{2 h_{T} \hat{\lambda}_{F} m_{F}}
$$

Where $m_{F}$ is the constant derivative level related to phase $\mathrm{F}$ and can also be calculated from the lines in the semilog graph for each analytical two phase case.

In table 6.3 , the single phase flow cases approximated equivalent permeabilities were calculated for their first region of permeability and compared to its respective real equivalent permeabilities and also, the two phase flow cases approximated equivalent permeabilities were calculated for their region filled with water phase $(F=w)$. For both cases, a comparison was made to its respective real equivalent permeabilities for these regions and the percentage errors were calculated for all cases:

\begin{tabular}{|l|l|l|l|}
\hline Case & $k_{e q}$ real $(\mathrm{mD})$ & $k_{e q}$ estimated $(\mathrm{mD})$ & Error $(\%)$ \\
\hline$A_{1}$ & 1000 & 1004.28 & 0.43 \\
\hline$B_{1}$ & 90 & 109.84 & 22 \\
\hline$C_{1}$ & 500 & 504.37 & 0.87 \\
\hline$D_{1}$ & 100 & 102.59 & 2.59 \\
\hline$E_{1}$ & 100 & 102.59 & 2.59 \\
\hline$F_{1}$ & 500 & 502.33 & 0.47 \\
\hline$A_{2}$ & 100 & 99.74 & 0.26 \\
\hline$B_{2}$ & 500 & 500.37 & 0.07 \\
\hline$C_{2}$ & 500 & 500.18 & 0.03 \\
\hline$D_{2}$ & 500 & 500.35 & 0.07 \\
\hline$E_{2}$ & 633.33 & 629.64 & 0.58 \\
\hline$F_{2}$ & 200 & 197.04 & 1.48 \\
\hline
\end{tabular}

Table 6.3: Real and estimated equivalent permeability values and percentage error for all cases 
Case $B_{1}$, which considers a damaged region arround the wellbore, has a bigger error because the value of the damaged region radius was smaller than 1 implying that the first values of the derivative curves reflects already the non-damaged region, interfering in the equivalent permeability values. Besides that, the estimations for the other cases presented small errors $(<2.6 \%)$.

The semilog graphs for each case used to calculate the equivalent permeability are given below in tables 6.4 and 6.5 :
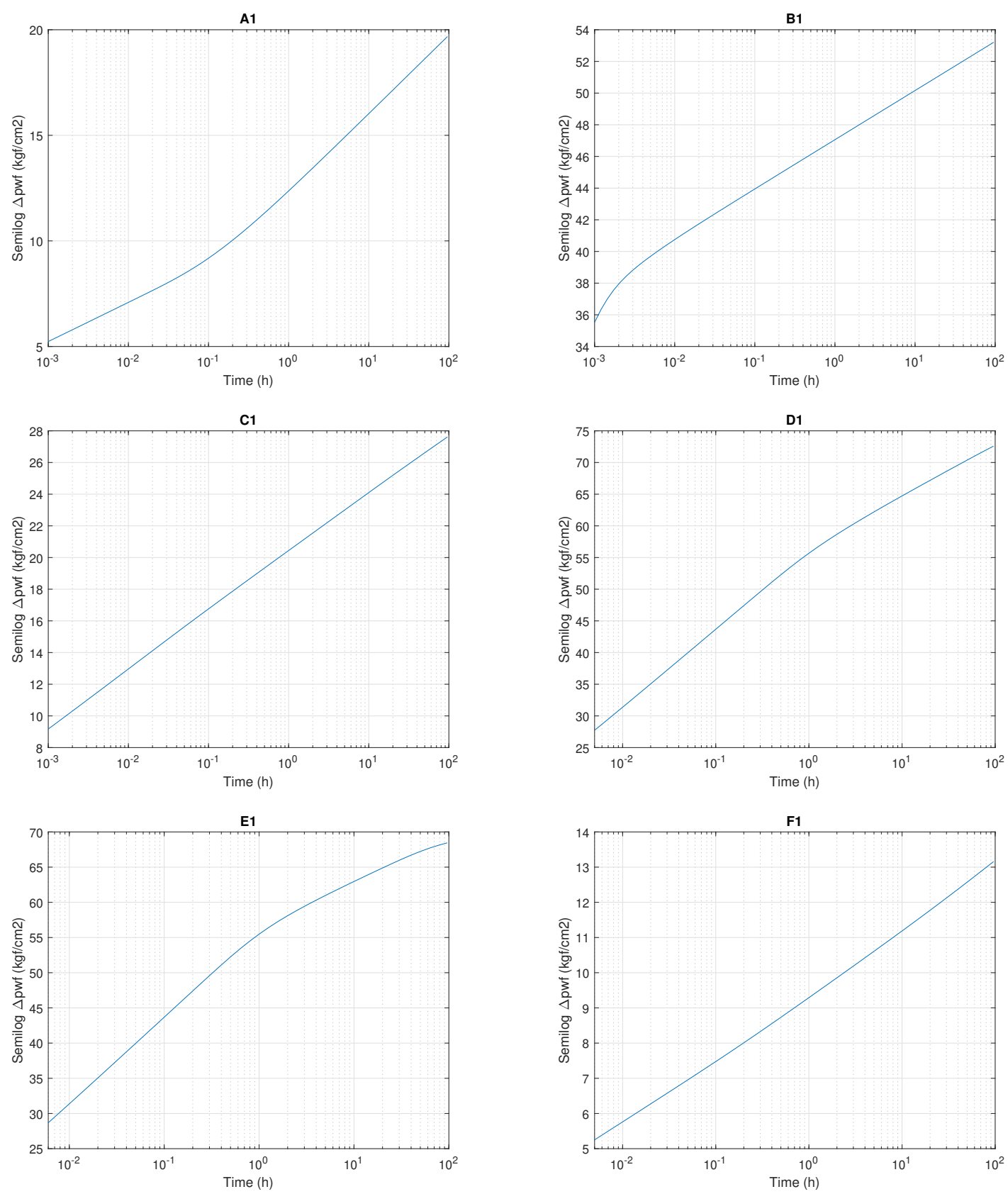

Table 6.4: Semilog graphs for the single phase flow cases 

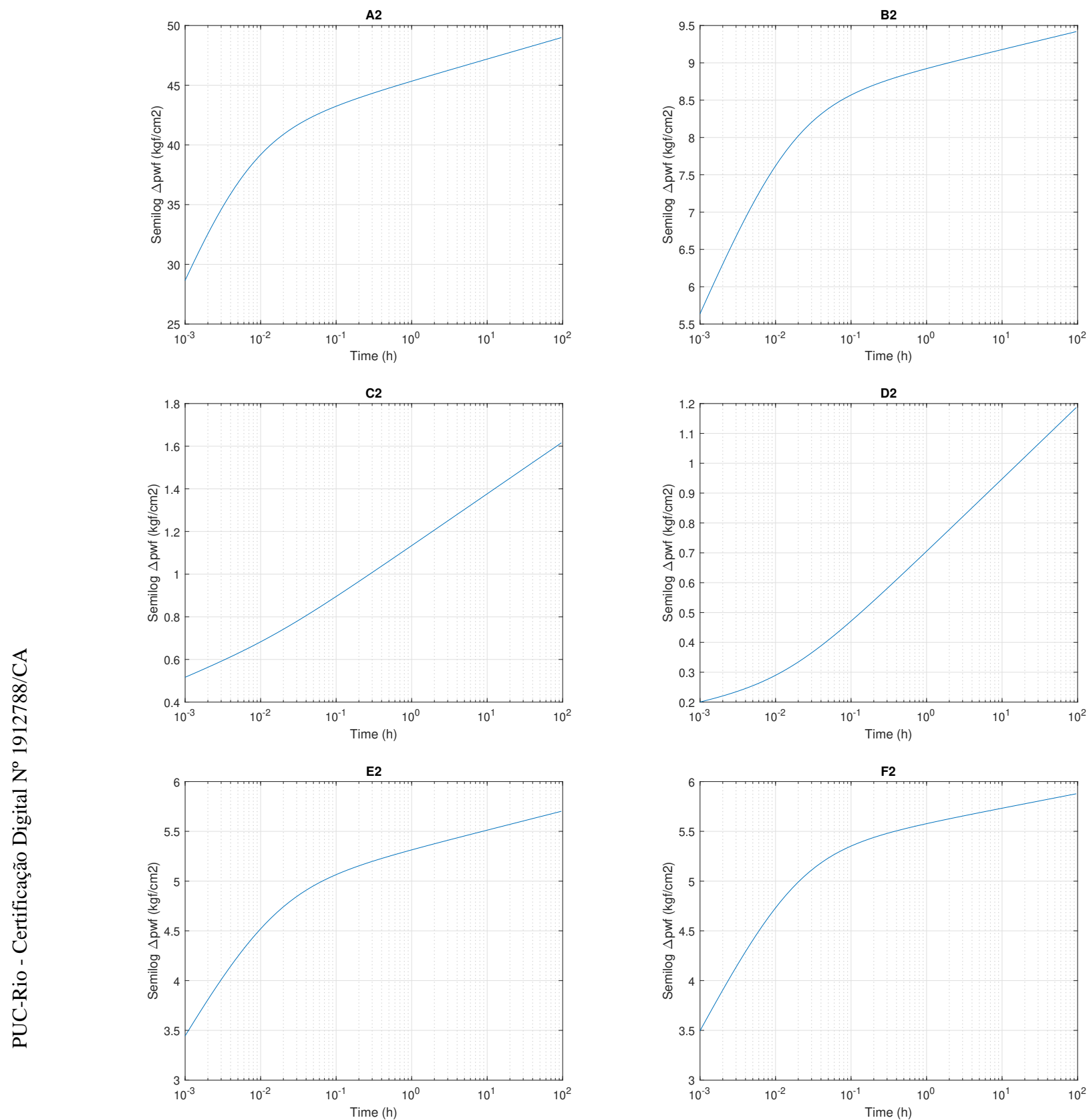

Table 6.5: Semilog graphs for the two phase flow cases 


\section{Conclusions and Suggestions for Future Work}

Based on the analytical solution proposed by Ehlig-Economides and Joseph [9], this work proposed a formulation that combined the presence of formation crossflow with having different coupling regions of permeability along each layer for multilayered reservoirs under single phase flow. Furthermore, this solution was extended to a case considering formation crossflow in a multilayered reservoir under two phase fluid flow, which was the main goal of this work.

The model here suggested was applied on a variety of cases. Cases with equal and different radii of regions of permeability for the single phase flow, cases including a damaged region near the wellbore and cases including multilayered reservoir systems under two phase fluid flow. In that sense, the model here presented is quite robust and dynamic.

For all cases, a comparison was made between the analytical solution and numerical simulation. In chapter 6 , it was possible to compare the agreement of these two results. The pressure response, along with other features, such as equivalent permeability, showed a good agreement in the comparison for all cases.

However, for cases with a big permeability difference from one layer to its adjacent one, the models presented show a bigger difference between the analytical and numerical responses. That may be because the formation crossflow simulation in the numerical simulator is not modeled exactly like in this work.

In the future, one work suggestion would be to explore more the impact of the formation crossflow for reservoirs with big differences of permeability from one layer to another, for example, the first layer with a range of permeabilities going from $100 \mathrm{mD}$ to $300 \mathrm{mD}$ and the second layer from $1500 \mathrm{mD}$ to $1800 \mathrm{mD}$.

Another suggestion is to consider the skin effects and multiple regions of permeability for each layer for a two phase fluid flow model. For these cases, there would be more coefficients of semipermeability, and the method to find the coupling conditions and calculating the $\sigma$ values would have to be more elaborated. Besides that, storage effect could be considered for single and two phase flow models. The present work gives all the support for such suggestions. 


\section{Bibliography}

[1] ANTOSIEWICZ, H.. Bessel functions of fractional order. Handbook of mathematical functions, 1970.

[2] AL GAHTANI, A.; YAHAYA, A. U. ; OTHERS. Field application of composite reservoir model on non-unit mobility flow. In: SPE/DGS SAUDI ARABIA SECTION TECHNICAL SYMPOSIUM AND EXHIBITION. Society of Petroleum Engineers, 2010.

[3] BARRETO, A. B. J.; PERES, A. M.; PIRES, A. P. ; OTHERS. Water injectivity tests on multilayered oil reservoirs. In: BRASIL OFFSHORE. Society of Petroleum Engineers, 2011.

[4] BonafÉ, M. F.; BRAGA, A. ; BARReto, A. B.. Approximate solution for pressure behavior during a multiple rate injectivity test. Journal of Petroleum Exploration and Production Technology, p. 1-14, 2020.

[5] BOURDET, D.; OTHERS. Pressure behavior of layered reservoirs with crossflow. In: SPE CALIFORNIA REGIONAL MEETING. Society of Petroleum Engineers, 1985.

[6] BRATVOLD, R. B.; HORNE, R. N. ; OTHERS. Analysis of pressure-falloff tests following cold-water injection. SPE Formation Evaluation, 5(03):293302, 1990.

[7] BUCKLEY, S. E.; LEVERETT, M. ; OTHERS. Mechanism of fluid displacement in sands. Transactions of the AIME, 146(01):107-116, 1942.

[8] CLOSMANN, P.; RATLIFF, N. ; OTHERS. Calculation of transient oil production in a radial composite reservoir. Society of Petroleum Engineers Journal, 7(04):355-358, 1967.

[9] EHLIG-ECONOMIDES, C. A.; JOSEPH, J. ; OTHERS. A new test for determination of individual layer properties in a multilayered reservoir. SPE Formation Evaluation, 2(03):261-283, 1987.

[10] LeFKOVITS, H.; HAZEBROEK, P.; ALlen, E.; MATTHEWS, C. ; OTHERS. A study of the behavior of bounded reservoirs composed of stratified layers. Society of Petroleum Engineers Journal, 1(01):43-58, 1961. 
[11] LF BITTEnCOURT NetO, J.; VIEIRA BELA, R.; PESCO, S.; BARRETO, A. ; OTHERS. Laplace domain pressure behavior solution for multilayered composite reservoirs. In: SPE LATIN AMERICAN AND CARIBBEAN PETROLEUM ENGINEERING CONFERENCE. Society of Petroleum Engineers, 2020.

[12] LF BitTencourt NetO, J.; VIEIRA BELA, R.; PESCO, S.; BARRETO, A. ; OTHERS. Pressure behavior during injectivity tests-a composite reservoir approach. In: SPE LATIN AMERICAN AND CARIBBEAN PETROLEUM ENGINEERING CONFERENCE. Society of Petroleum Engineers, 2020.

[13] PRIJAMBODO, R.; RAGHAVAN, R.; REYNOLDS, A. ; OTHERS. Well test analysis for wells producing layered reservoirs with crossflow. Society of Petroleum Engineers Journal, 25(03):380-396, 1985.

[14] STEHFEST, H.. Algorithm 368: Numerical inversion of laplace transforms [d5]. Communications of the ACM, 13(1):47-49, 1970.

[15] SUN, H.; GAO, C.. Well Test Analysis for Multilayered Reservoirs with Formation Crossflow. Gulf Professional Publishing, 2017.

[16] TARIQ, S. M.; RAMEY JR, H. J. ; OTHERS. Drawdown behavior of a well with storage and skin effect communicating with layers of different radii and other characteristics. In: SPE ANNUAL FALL TECHNICAL CONFERENCE AND EXHIBITION. Society of Petroleum Engineers, 1978. 
A

\section{Pressure solution in the Laplace's field}

In order to solve the problems presented in this work, the solution is first given in the Laplace field in terms of Bessel's functions. The properties used both on Laplace transforms and on Bessel's functions are described in this appendix section.

The Stehfest Algorithm[14], which is also described in this appendix section, is then used to find the solutions in the real field.

\section{A.1}

\section{Some Properties on the Laplace's Transform}

Consider a real function $f(t)$. The Laplace's Transform of this function is defined as:

$$
\mathcal{L}|f(t)|=\int_{0}^{\infty} f(t) e^{-u t} d t
$$

Where $u$ is the Laplace's variable. The Laplace's Transform will be denoted, in this work, by the following notation:

$$
\mathcal{L}|f(t)|=\bar{f}(u)
$$

Some useful operational properties on the Laplace's Transform are given below:

$$
\begin{aligned}
& \text { 1) } \mathcal{L}\left|\frac{d f(t)}{d t}\right|=\mathcal{L}\left|f^{\prime}(t)\right|=u f^{\prime}(u)-f(0) \\
& \text { 2) } \quad \mathcal{L}\left|\frac{\partial f(x, t)}{\partial x}\right|=\frac{\partial \bar{f}(x, u)}{\partial x}
\end{aligned}
$$

3)

$$
\mathcal{L}|f * g|=\mathcal{L}|f| \mathcal{L}|g|=\bar{f}(u) \bar{g}(u) \Leftrightarrow \mathcal{L}|\bar{f}(u) \bar{g}(u)|=f * g
$$




\section{A. 2}

\section{Some Properties on the Bessel's Functions}

Consider the following differential equation:

$$
\frac{d^{2} y}{d z^{2}}+\frac{1}{z} \frac{d y}{d z}-\left(1-\frac{v^{2}}{z^{2}}\right) y=0
$$

The general solution for this equation is:

$$
y=A K_{v}(z)+B I_{v}(z)
$$

Where $v$ is a real number and $z$ can be a complex number, $A$ and $B$ are constants, $I_{v}(z)$ is a modified Bessel's function of first kind and order v, $K_{v}(z)$ is a modified Bessel's function of second kind and order v. In addition, $I_{v}(z)$ and $K_{v}(z)$ are positive and real functions when $v>1$ and $z>0$ and they are also, linearly independent for all values of $v$.

The problems considered in this work fall on a particular case of equation (A2.1):

$$
\frac{d^{2} y}{d z^{2}}+\frac{1}{z} \frac{d y}{d z}-y=0
$$

Which has the following solution:

$$
y=A K_{0}(z)+B I_{0}(z)
$$

The following properties apply on functions $I_{0}(z), I_{1}(z), K_{0}(z)$ and $K_{1}(z)$, and can be illustrated in the figure below: 


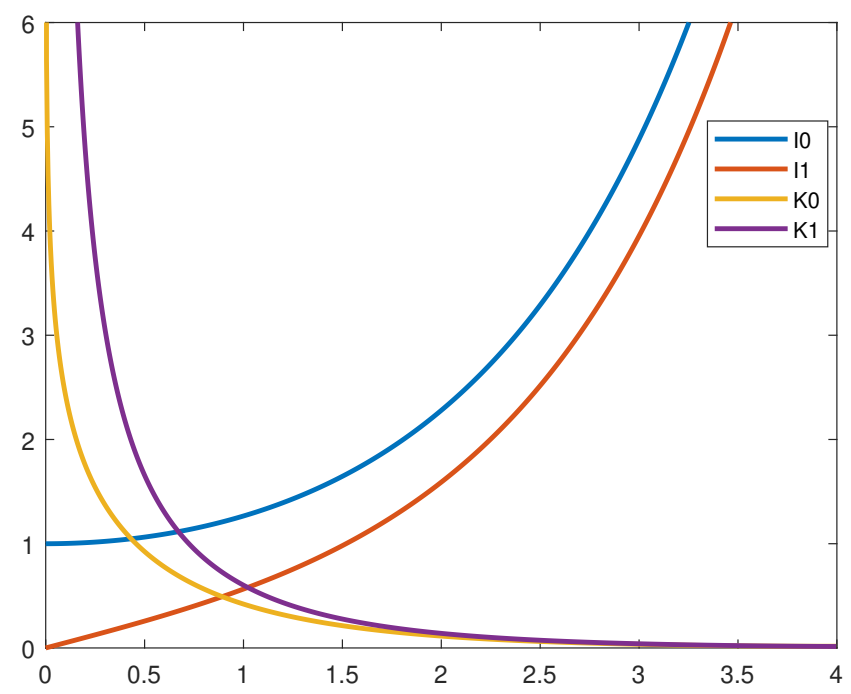

Figure A.1: Modified Bessel's functions

1) $\lim _{x \rightarrow \infty} K_{0}(x)=0$

2) $\quad \lim _{x \rightarrow 0} K_{0}(x)=\infty$

3) $\quad \lim _{x \rightarrow \infty} K_{1}(x)=0$

4) $\quad \lim _{x \rightarrow 0} K_{1}(x)=\infty$

5) $\quad \lim _{x \rightarrow 0} I_{0}(x)=1$

6) $\quad \lim _{x \rightarrow \infty} I_{0}(x)=\infty$ 


$$
\begin{aligned}
& \text { 7) } \lim _{x \rightarrow 0} I_{1}(x)=0 \\
& \text { 8) } \lim _{x \rightarrow \infty} I_{1}(x)=\infty \\
& \text { 9) } I_{0}^{\prime}(x)=I_{1}(x) \\
& \text { 10) } \quad K_{0}^{\prime}(x)=-K_{1}(x)
\end{aligned}
$$

Polynomials approximations of the modified Bessel's functions can be calculated in [1].

\section{A.3}

\section{Stehfest's Algorithm}

The pressure variation response, which was calculated in the Laplace domain for all problems proposed in this work, can be obtained in the real field through a numerical inversion. In this work, the inversion used is the one given by the Stehfest Algorithm[14]. Which is given by:

$$
f a(t) \approx \frac{\log 2}{t} \sum_{j=1}^{N} V_{j} \bar{f}\left(\frac{\log 2}{t} j\right)
$$

Where the terms $V_{j}$ are computed using the following:

$$
V_{j}=(-1)^{j+\frac{N}{2}} \sum_{k=\operatorname{int}\left(\frac{j+1}{2}\right)}^{\min \left(j, \frac{N}{2}\right)}\left[\frac{k^{1+\frac{N}{2}}(2 k) !}{\left(\frac{N}{2}-k\right) !(k !)^{2}(j-k) !(2 k-j) !}\right]
$$

Where $N$ is a parameter chosen by the user and should be even. A recommended interval is $10 \leq N \leq 18$.

Take, for example, the solution in the Laplace field for the problem of onephase flow to finite dimensionless radius: 


$$
\bar{p}_{D}\left(r_{D}, u\right)=\frac{K_{0}\left(r_{D} \sqrt{u}\right)}{u^{\frac{3}{2}} K_{1}(\sqrt{u})}
$$

In the real field, equation (A3.3) can be written as the following:

$$
p_{D}\left(r_{D}, u\right)=\frac{\log 2}{t} \sum_{j=1}^{N} V_{j} \bar{p}_{D}\left(r_{D}, u\right)
$$

And $u$ is given by:

$$
u=\frac{\log 2}{t_{D}} j
$$


B

\section{Multilayered Case Under Single Phase Flow with Formation Crossflow and a Single Region of Permeability per Layer}

For this problem, the mathematical formulation was derived from [9]. This case, which considers a single region of permeability for each layer, gives support for the mathematical formulation presented in this work for a radially composed reservoir.

First, consider the dimensionless variables:

$$
\begin{aligned}
r_{D} & =\frac{r}{r_{w}} \\
p_{j D} & =\frac{2 \pi \overline{k h}}{q \mu}\left(p_{i}-p_{j}\right) \\
t_{D} & =\frac{\overline{k h}}{\overline{\phi h} \mu c_{t} r_{w}^{2}} t \\
\overline{k h} & =\sum_{j=1}^{n}(k h)_{j} \\
\overline{\phi h} & =\sum_{j=1}^{n}(\phi h)_{j} \\
\kappa_{j} & =\frac{(k h)_{j}}{\overline{k h}} \\
\omega_{j} & =\frac{(\phi h)_{j}}{\overline{\phi h}} \\
\lambda_{j} & =\frac{X_{j} r_{w}^{2}}{\overline{k h}}
\end{aligned}
$$

The following hypothesis (CCL) are given for this problem:

$$
\begin{aligned}
& p_{1}\left(r_{w}, t\right)=p_{2}\left(r_{w}, t\right)=\ldots=p_{n}\left(r_{w}, t\right)=p_{w} \\
& q_{B}=-2 \pi r_{w} \sum\left(\frac{k_{j} h_{j}}{\mu}\right) \frac{\partial p_{w}}{\partial \overline{r_{D}}}
\end{aligned}
$$


In the Laplace domain the partial differential equation is:

$$
\kappa_{j} \nabla^{2} \bar{p}_{j D}=\omega_{j} u \bar{p}_{j D}+\lambda_{j-1 D}\left(\bar{p}_{j D}-\bar{p}_{j-1 D}\right)-\lambda_{j D}\left(\bar{p}_{j+1 D}-\bar{p}_{j D}\right)
$$

The general solution for pressure in the infinite radial case in the Laplace field is:

$$
\bar{p}_{j D}=A_{j} K_{0}\left(\sigma r_{D}\right)
$$

Replacing (B.12) in (B.11) for $j=1, \ldots, n$ :

$$
A_{j} K_{0}\left(\sigma r_{D}\right)\left(\kappa_{j} \sigma^{2}-\omega_{j} u+\lambda_{j D}\right)=\lambda_{j-1 D}\left(A_{j} K_{0}\left(\sigma r_{D}\right)-A_{j-1} K_{0}\left(\sigma r_{D}\right)\right)-\lambda_{j D}\left(A_{j+1} K_{0}\left(\sigma r_{D}\right)\right)
$$

Rearranging equation (B.0.13), the following linear system to find the values of $\sigma$ is set up:

$$
\left\{\begin{array}{l}
j=1: \quad\left(\omega_{1} u+\lambda_{1}-\kappa_{1} \sigma^{2}\right) A_{1}-\lambda_{1} A_{2}=0 \\
j=2: \quad-\lambda_{1} A_{1}+\left(\omega_{2} u+\lambda_{1}+\lambda_{2}-\kappa_{2} \sigma^{2}\right) A_{2}-\lambda_{2} A_{3}=0 \\
\vdots \\
j=n: \quad-\lambda_{n-1} A_{n-1}+\left(\omega_{n} u+\lambda_{n-1}+\lambda_{n}-\kappa_{n} \sigma^{2}\right) A_{n}=0
\end{array}\right.
$$

$K_{0}\left(\sigma_{k} r_{D}\right) \neq 0$, and also, the coefficients $A_{j}$ must be different than zero, since the trivial solution to the pressure is not wanted. Therefore, the matrix below $a_{j k}$ must be singular, that is, not invertible and its determinant must vanish:

$$
a_{j k}= \begin{cases}\lambda_{j-1}, & \text { for } k=j-1 ; j>1, \\ \kappa_{j} \sigma^{2}-\omega_{j} u-\lambda_{j-1}-\lambda_{j}, & \text { for } k=j, \\ \lambda_{j}, & \text { for } k=j+1 ; j<n, \\ 0, & \text { for } k \neq j-1, j, \text { or } j+1 .\end{cases}
$$


It is possible to identify a relation between calculating the determinant of $a_{j k}$ to find $\sigma$ and calculating the eigenvalues $\kappa_{j} \sigma^{2}$ of matrix $a_{j k}^{\prime}$ given by (B.0.16):

$$
a_{j k}^{\prime}= \begin{cases}-\lambda_{j-1}, & \text { for } k=j-1 ; j>1, \\ -\omega_{j} u+\lambda_{j-1}+\lambda_{j}, & \text { for } k=j \\ -\lambda_{j}, & \text { for } k=j+1 ; j<n \\ 0, & \text { for } k \neq j-1, j, \text { or } j+1 .\end{cases}
$$

The pressure solution is given by:

$$
\bar{p}_{j D}=\sum_{k=1}^{n} A_{j}^{k} K_{0}\left(\sigma^{k} r_{D}\right)
$$

It is possible to see that it remains to find the coefficients $A_{j}^{k}$. After finding the values of $\sigma$, a recursive formula from the system above is given, where the values of each $A_{j}^{k}$ to $j=2, \ldots, n$ as a function of $A_{1}^{k}$ is given:

$$
\left\{\begin{array}{l}
A_{2}^{k}=\frac{\left(\omega_{1} u+\lambda_{1}-\kappa_{1} \sigma_{k}^{2}\right)}{\lambda_{1}} A_{1}^{k} \\
A_{3}^{k}=\frac{1}{\lambda_{2}}\left[-\lambda_{1} A_{1}^{k}+\left(\omega_{2} u+\lambda_{1}+\lambda_{2}-\kappa_{2} \sigma_{k}^{2}\right) A_{2}^{k}\right] \\
\vdots \\
A_{n}^{k}=\frac{1}{\lambda_{n-1}}\left[-\lambda_{n-2} A_{n-2}^{k}+\left(\omega_{n-1} u+\lambda_{n-2}+\lambda_{n-1}-\kappa_{n-1} \sigma_{k}^{2}\right) A_{n-1}^{k}\right]
\end{array}\right.
$$

Now using Darcy's Law:

$$
\bar{q}_{D}=-\frac{1}{u}=-\kappa_{j} \frac{\partial \bar{p}_{j}}{\partial r_{D}}
$$

Considering the following hypothesis: $\bar{p}_{1}=p_{w f}$ :

$$
\frac{1}{u \sum_{j=1}^{n} \kappa_{j}}=\frac{\partial\left(\sum_{k=1}^{n} A_{1}^{k} \alpha_{j}^{k} K_{0}\left(\sigma_{k} r_{D}\right)\right)}{\partial \overline{r_{D}}}
$$


The following $\mathrm{n}$ relations are given, which lead to a second linear system to find the pressure coefficients $A_{1}^{k}$ :

Flow rate in the well, where $r_{D}=1$, for the first system equation:

$$
\bar{q}_{D}=\frac{1}{u \sum_{j=1}^{n} \kappa_{j}}=\sum_{k=1}^{n_{k}} \sigma_{k} A_{1}^{k} \alpha_{j}^{k} K_{1}\left(\sigma_{k}\right)
$$

Well pressure for the other $n-1$ equations:

$$
0=\sum_{k=1}^{n_{k}} A_{j-1}^{k} K_{0}\left(\sigma_{k}\right)-\sum_{k=1}^{n_{k}} A_{j}^{k} K_{0}\left(\sigma_{k}\right)=\sum_{k=1}^{n_{k}} K_{0}\left(\sigma_{k}\right)\left(1-\alpha_{j}^{k}\right) A_{1}^{k}
$$

Then, the system is given by:

$$
\left[\begin{array}{cccc}
\alpha_{1}^{2} \sigma_{1} K_{1}\left(\sigma_{1}\right) & \alpha_{1}^{2} \sigma_{2} K_{1}\left(\sigma_{2}\right) & \ldots & \alpha_{1}^{n k} \sigma_{n k} K_{1}\left(\sigma_{n k}\right) \\
\left(1-\alpha_{2}^{1}\right) K_{0}\left(\sigma_{1}\right) & \left(1-\alpha_{2}^{2}\right) K_{0}\left(\sigma_{2}\right) & \ldots & \left(1-\alpha_{2}^{n k}\right) K_{0}\left(\sigma_{n k}\right) \\
\vdots & & & \vdots \\
\left(1-\alpha_{n k}^{1}\right) K_{0}\left(\sigma_{1}\right) & \left(1-\alpha_{n k}^{2}\right) K_{0}\left(\sigma_{2}\right) & \ldots & \left(1-\alpha_{n k}^{n k}\right) K_{0}\left(\sigma_{n k}\right)
\end{array}\right]\left[\begin{array}{c}
A_{1}^{1} \\
A_{1}^{2} \\
\vdots \\
A_{1}^{n k}
\end{array}\right]=\left[\begin{array}{c}
\frac{1}{u \sum \kappa_{j}} \\
0 \\
\vdots \\
0
\end{array}\right]
$$

Where each $\alpha$ above is defined using the coefficients relation in (B.18). After calculating the coefficients, it is possible to calculate the pressure:

$$
\bar{p}=\sum_{k=1}^{n k} A_{1}^{k} \alpha_{1}^{k} K_{0}\left(\sigma_{k}\right)
$$




\section{C \\ Semipermeability Coefficients Number}

Let $\alpha$ be the number of intersections between the regions of 2 Layers.

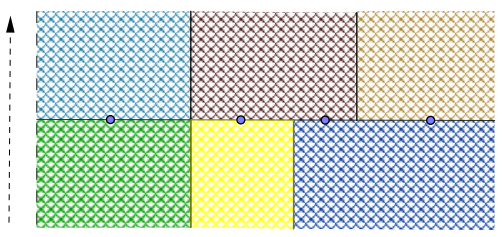

Figure C.1: Case where $\alpha=4$

Let $M_{1}, \ldots, M_{m}$ be the regions of the under layer, each one of these regions has radius $m_{1}, \ldots, m_{m}$, respectively. Likewise, let $N_{1}, \ldots, N_{n}$ and $n_{1}, \ldots, n_{n}$. Notice that $n_{1}+\ldots+n_{n}=m_{1}+\ldots+m_{m}$.

Now, $0=n_{0}<n_{1}<n_{1}+n_{2}<\ldots<n_{1}+\ldots+n_{n}$. Let $a_{i}=n_{1}+\ldots+n_{i}$.

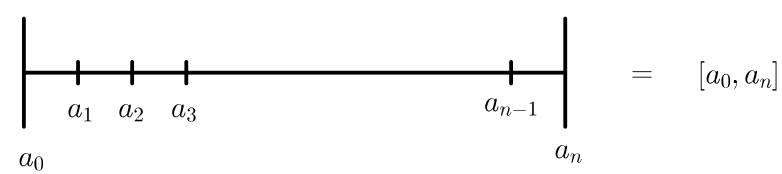

Similarly, $0=m_{0}<m_{1}<m_{1}+m_{2}<\ldots<m_{1}+\ldots+m_{m}$. Let $b_{j}=m_{1}+\ldots+m_{j}$.

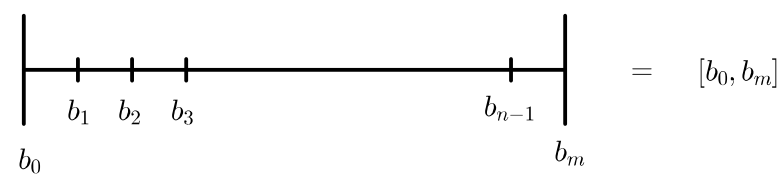

Hence, $\alpha$ is given by the number of subsets obtained whilst superposing $\left[a_{0}, a_{n}\right]$ and $\left[b_{0}, b_{m}\right] .\left[a_{0}, a_{n}\right]$ has $n$ partitions. When superposing those sets there will be a new one $\left[a_{0}, a_{n}\right]^{\prime}$ with an extra $m-1$ partitions. By hypothesis, there are $s$ values $i, j$ such that $a_{i}=b_{j}$. In that way, there will actually be $m-1-s$ new partitions in the interior of $\left[a_{0}, a_{n}\right]^{\prime}$. Then, $\alpha=n+m-1-s$, that is, there are $n+m-1-s$ subsets. 